\title{
छூ \\ Commissioning experience and beam physics measurements at the SwissFEL Injector Test Facility
}

T. Schietinger, M. Pedrozzi, M. Aiba, V. Arsov, S. Bettoni, B. Beutner, M. Calvi, P. Craievich, M. Dehler, F. Frei, R. Ganter, C. P. Hauri, R. Ischebeck, Y. Ivanisenko, M. Janousch, M. Kaiser, B. Keil, F. Löhl, G. L. Orlandi, C. Ozkan Loch, P. Peier, E. Prat, J.-Y. Raguin, S. Reiche, T. Schilcher, P. Wiegand, E. Zimoch, D. Anicic, D. Armstrong, M. Baldinger, R. Baldinger, A. Bertrand, K. Bitterli, M. Bopp, H. Brands, H. H. Braun, M. Brönnimann, I. Brunnenkant, P. Chevtsov, J. Chrin, A. Citterio, M. Csatari Divall, M. Dach, A. Dax, R. Ditter, E. Divall, A. Falone, H. Fitze, C. Geiselhart, M. W. Guetg, F. Hämmerli, A. Hauff, M. Heiniger, C. Higgs, W. Hugentobler, S. Hunziker, G. Janser, B. Kalantari, R. Kalt, Y. Kim, W. Koprek, T. Korhonen,

R. Krempaska, M. Laznovsky, S. Lehner, F. Le Pimpec, T. Lippuner, H. Lutz, S. Mair, F. Marcellini, G. Marinkovic, R. Menzel, N. Milas, T. Pal, P. Pollet, W. Portmann, A. Rezaeizadeh, S. Ritt, M. Rohrer, M. Schär, L. Schebacher, St. Scherrer, V. Schlott, T. Schmidt, L. Schulz, B. Smit, M. Stadler, B. Steffen, L. Stingelin, W. Sturzenegger, D. M. Treyer, A. Trisorio, W. Tron, C. Vicario, R. Zennaro, and D. Zimoch

Paul Scherrer Institut, CH-5232 Villigen PSI, Switzerland

(Received 9 June 2016; published 26 October 2016)

The SwissFEL Injector Test Facility operated at the Paul Scherrer Institute between 2010 and 2014, serving as a pilot plant and test bed for the development and realization of SwissFEL, the x-ray FreeElectron Laser facility under construction at the same institute. The test facility consisted of a laser-driven rf electron gun followed by an S-band booster linac, a magnetic bunch compression chicane and a diagnostic section including a transverse deflecting rf cavity. It delivered electron bunches of up to $200 \mathrm{pC}$ charge and up to $250 \mathrm{MeV}$ beam energy at a repetition rate of $10 \mathrm{~Hz}$. The measurements performed at the test facility not only demonstrated the beam parameters required to drive the first stage of an FEL facility, but also led to significant advances in instrumentation technologies, beam characterization methods and the generation, transport and compression of ultralow-emittance beams. We give a comprehensive overview of the commissioning experience of the principal subsystems and the beam physics measurements performed during the operation of the test facility, including the results of the test of an in-vacuum undulator prototype generating radiation in the vacuum ultraviolet and optical range.

DOI: 10.1103/PhysRevAccelBeams.19.100702

\section{INTRODUCTION}

The Paul Scherrer Institute (PSI) is currently constructing SwissFEL, an X-ray Free-Electron Laser (FEL) designed to produce coherent, ultra-bright and ultra-short photon pulses in the wavelength range between 0.1 and $7 \mathrm{~nm}$, with first pilot experiments scheduled for late 2017 $[1,2]$. During the preparation phase of SwissFEL, PSI designed, built and operated a $250-\mathrm{MeV}$ photoinjector, the SwissFEL Injector Test Facility (SITF) [3]. Its main purposes consisted in the detailed study of the generation, acceleration and time compression of high-brightness electron beams needed to drive a compact SASE (selfamplified spontaneous emission) FEL and in providing a

*thomas.schietinger@psi.ch

Published by the American Physical Society under the terms of the Creative Commons Attribution 3.0 License. Further distribution of this work must maintain attribution to the author(s) and the published article's title, journal citation, and DOI. platform for the development and test of accelerator and undulator components or systems planned for SwissFEL.

Several FEL test facilities with varying objectives have operated at other labs, going back to the TESLA Test Facility (TTF) at DESY [4,5], the photoinjector test facility at DESY, Zeuthen (PITZ) [6], and the SLAC Gun Test Facility (GTF) [7], followed by the SPARC photoinjector $[8,9]$ at INFN Frascati, the Shanghai Deep Ultraviolet FreeElectron Laser source (SDUV-FEL) [10], the SPring8 Compact SASE Source (SCSS) test accelerator at RIKEN [11,12], or, more recently, the PAL-XFEL injector test facility at the Pohang Accelerator Laboratory (PAL) $[13,14]$, and further facilities are being constructed in this context, e.g., the CLARA test facility at Daresbury [15]. Moreover, SLAC's Next Linear Collider Test Accelerator (NLCTA) [16] has been used extensively for FEL studies (see, e.g., Ref. [17]), and reports on commissioning experience have been given for the injector of the Linac Coherent Light Source (LCLS) at SLAC [18] and the FERMI project at Elettra Sincrotrone Trieste [19]. 
The primary focus of the SITF lied on the systematic characterization and optimization of the electron source up to and including the first bunch compression stage. The beam optimization was accomplished through the tuning of all relevant parameters including the gun laser, cathode properties, radio frequency (rf) acceleration and beam optics setup. The aim of these efforts was to generate, and preserve after magnetic bunch compression, a highbrightness beam fulfilling the stringent requirements for driving an FEL of compact and thus cost-effective design like SwissFEL. As the verification of such a highbrightness beam requires advanced beam instrumentation as well as excellent beam characterization methods, much emphasis was put on both of these areas at the SITF.

The SITF operated between March 2010 and October 2014, with installation and repair breaks in between, in various configurations allowing for a wide range of beam measurements and component tests. In the present report we give a review of the commissioning experience of the main subsystems and summarize the beam physics measurements performed at the test facility. We describe the design and general layout of the facility in Sec. II, followed by a chronological account of the various operating phases in Sec. III. Section IV contains commissioning and performance reviews of the main subsystems, with particular emphasis on the gun laser, the rf systems and the electron beam diagnostics. In Sec. V we present some diagnostics developments performed at the SITF in view of the realization of SwissFEL. In the following two sections we report on our beam development work, describing our methods and procedures (Sec. VI) as well as presenting and discussing the results of our measurements (Sec. VII). Section VIII is dedicated to the undulator prototype test performed at the SITF. In Sec. IX we summarize our work and give a brief outlook on SwissFEL.

\section{MACHINE LAYOUT AND DESIGN CONSIDERATIONS}

The SITF was conceived as a split rf photoinjector followed by a booster linac, a magnetic bunch compression chicane and a diagnostic section. The facility was housed in a dedicated building at PSI, the laboratory hall WLHA, which was constructed in the years 2008 and 2009 and includes a shielding tunnel for the beam line.

The design of the facility [20] benefited from several conceptual and experimental insights in injector design gained in the last three decades. In particular the design is based on the concepts of emittance compensation [21] and invariant envelope matching [22], as well as the effective working point found by Ferrario et al. [23] during the redesign of the Linac Coherent Light Source (LCLS) and later confirmed by measurements performed at the SPARC photoinjector [24] and, eventually, by the successful operation of the LCLS facility $[18,25]$. Further guiding principles were given by the need for an azimuthally symmetric beam at low energy to provide an equal space-charge density in the vertical and horizontal plane, the preference for a relatively low bunch charge (compared to earlier x-ray FEL designs), allowing for a smaller laser spot size on the cathode and thus resulting in a smaller thermal emittance contribution, the minimization of nonlinearities and nonuniformities in the bunch slice parameters (good slice matching) and the aim for a small $\beta$-function to minimize chromatic effects in the focusing quadrupole magnets.

A schematic representation of the layout of the facility in its initial configuration is given in Fig. 1. The electron source is a laser-driven S-band ( $3 \mathrm{GHz}$ ) rf gun. During the first four years of operation, until April 2014, electrons were produced with an rf gun originally built for the CLIC test facility (CTF) at CERN, the CTF gun No. V [26,27], kindly lent by CERN to PSI. Later, the PSI-built SwissFEL rf gun [28] was installed and used. In both cases, the electrons were extracted by a laser pulse from an exchangeable copper cathode plug (at later stages in some cases coated with cesium telluride) and accelerated to an initial momentum of $7.1 \mathrm{MeV} / c$. From 2013 onward, the exchange of cathodes was facilitated by a load-lock chamber mounted onto the rf gun. Immediately after the gun, a movable solenoid magnet provides initial focusing and is used to optimize the emittance implementing the emittance compensation technique along the following $3 \mathrm{~m}$ long drift. The gun solenoid includes two weak, individually tunable quadrupole magnets (normal and skew) to correct potential quadrupole terms in the solenoid field [29] (see also Ref. [30] for a theoretical description of the correction scheme). The ensuing drift accommodates various low-energy beam diagnostics including a spectrometer arm at $30^{\circ}$ deflection angle for the measurement of beam energy and energy spread. The main accelerating section consists of four S-band traveling-wave structures boosting the beam energy to a nominal value of $270 \mathrm{MeV}$. Each of the structures is surrounded by four solenoid magnets for additional symmetric transverse focusing. The solenoids are each $75 \mathrm{~cm}$ long and provide magnetic fields up to $100 \mathrm{mT}$. The two downstream S-band structures are simultaneously used to generate, by off-crest acceleration, the energy chirp necessary for the longitudinal compression of the electron bunches in the magnetic four-dipole chicane after the booster. Since the energy chirp generated in this way results in a curved longitudinal phase-space distribution, a fourth-harmonic cavity (X-band, $12 \mathrm{GHz}$ ) is operated upstream of the bunch compressor for the linearization of the longitudinal phase space, thereby suppressing the development of peaks in the head and tail of the current profile of the compressed bunch. This linearization includes the compensation of second-order terms arising from the bunch compression process. Under normal conditions, the harmonic cavity has a net decelerating effect on the bunch centroid, reducing the beam energy to the 


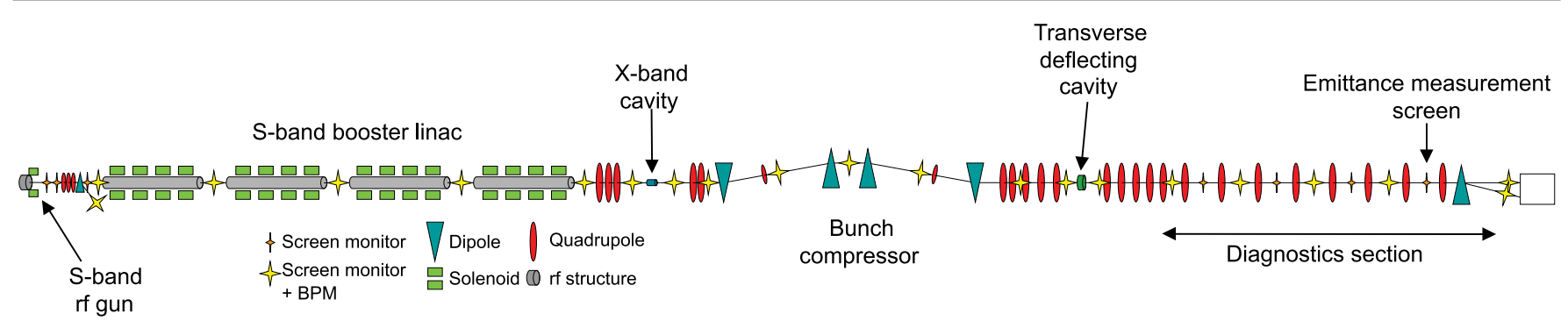

FIG. 1. Schematic layout of the SwissFEL Injector Test Facility (original configuration, not to scale). The total length of the beam line is approximately $60 \mathrm{~m}$.

nominal final energy of $250 \mathrm{MeV}$. The matching of the beam optics to the design optics is accomplished with five quadrupole magnets upstream of the chicane. After the bunch compressor the beam enters an extensive diagnostic section optimized for bunch length and emittance measurements. An array of quadrupole magnets allows the implementation of numerous optics schemes, in particular to perform various kinds of optics-based measurements of emittance and Twiss parameters. The initial configuration of the diagnostics section, as depicted in Fig. 1, included a Focus-Drift-Defocus-Drift (FODO) channel for the measurement of emittance using multiple screens inside the FODO cells, as an alternative to single or multiple quadrupole scan techniques. The FODO option was lost in the course of the modification of the diagnostics beam line to accommodate an undulator prototype in early 2014. At this point, however, the quadrupole scan methods had already been firmly established as being both more robust and more precise. A vertically deflecting S-band rf cavity located after a few more matching quadrupoles beyond the bunch compressor can be used to streak the bunch, thus enabling the measurement of bunch length and slice parameters with a screen at a suitable distance. Immediately before the beam dump, a high-energy spectrometer, consisting of a deflecting dipole magnet ( $6^{\circ}$ bending angle), a straight and a dispersive section allows momentum and momentum spread of the final electron beam to be measured. It can also be used in combination with the transverse deflecting cavity for the detailed imaging of the longitudinal phase space. At various positions along the entire beam line, transverse beam profiles can be obtained by imaging the beam with insertable screens, consisting of crystals (YAG:Ce or LuAG:Ce) emitting scintillation light or thin metal foils emitting optical transition radiation (OTR). The beam orbit and charge transmission is monitored by a series of resonant stripline beam position monitors (BPMs). Additional cavity-based BPMs were installed for test purposes along the straight section of the high-energy spectrometer. A wall current monitor in the gun section provides a measurement of the absolute bunch charge with an uncertainty of $10 \%-15 \%$ and serves as a calibration for the BPM charge measurements. Additional information on the bunch charge can be obtained from a few prototype integrating current transformers installed along the beam line.

Figure 2 shows a typical beam optics setting, as it was applied for the main measurements related to beam characterization and optimization described in Sec. VII.

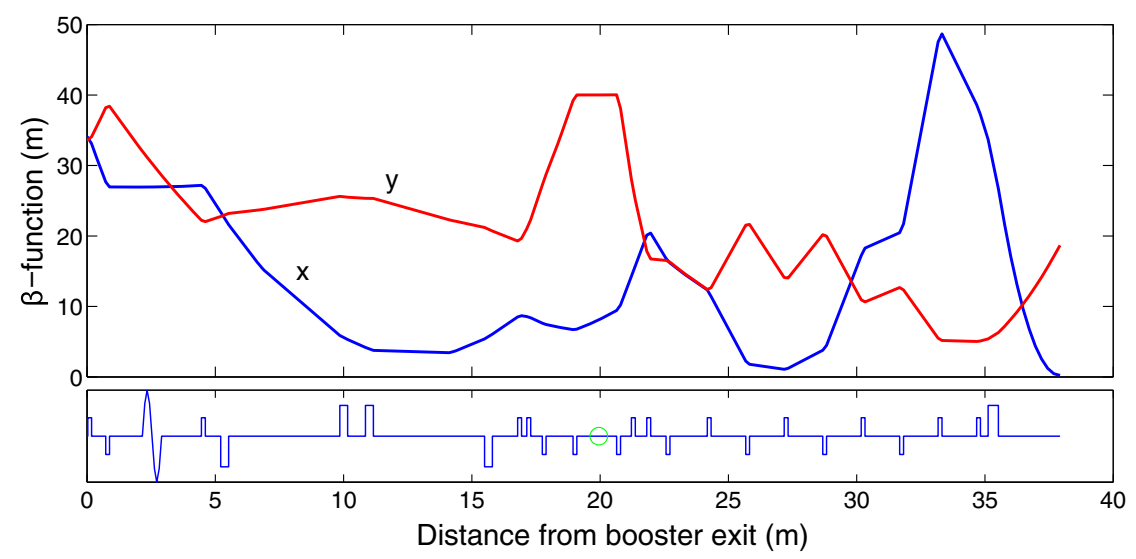

FIG. 2. Horizontal (blue) and vertical (red) $\beta$-functions for a typical beam optics setup, covering the beam line from the S-band booster exit down to the profile monitor at the high-energy spectrometer (upper panel). The corresponding magnetic lattice is shown in the lower panel, where the larger rectangular shapes indicate the locations of the main dipole (bending) magnets and the smaller those of quadrupole (focusing) magnets. The wave-like curve at $2.5 \mathrm{~m}$ represents the position of the X-band cavity. The green circle at $20 \mathrm{~m}$ marks the location of the rf deflector. 
TABLE I. Main design parameters of the SwissFEL Injector Test Facility for the high-charge mode (200 pC bunch charge), with comparison to typically realized values.

\begin{tabular}{|c|c|c|c|}
\hline Parameter & Design & & Realized \\
\hline Final beam energy $(\mathrm{MeV})$ & 250 & & $260^{\mathrm{a}}$ \\
\hline Repetition rate $(\mathrm{Hz})$ & 10 & & 10 \\
\hline Cathode quantum efficiency & $5 \times 10^{-5}(\mathrm{Cu})$ & & $\begin{array}{c}3 \times 10^{-4}(\mathrm{Cu}) \\
6 \times 10^{-2}\left(\mathrm{Cs}_{2} \mathrm{Te}\right)\end{array}$ \\
\hline Peak rf gun field at cathode (MV/m) & 100 & & $100^{\mathrm{b}}$ \\
\hline Intrinsic emittance contribution $(\mu \mathrm{m})$ & 0.195 & & $\approx 0.1$ \\
\hline \multicolumn{4}{|l|}{ Gun laser parameters } \\
\hline Spot diameter on cathode (mm) & & 1 & \\
\hline Pulse length (FWHM) (ps) & & 9.9 & \\
\hline Wavelength (nm) & & $257-280$ & \\
\hline \multicolumn{4}{|l|}{ Before compression: } \\
\hline Bunch length (rms) (ps) & 2.8 & & 3.8 \\
\hline Peak current (A) & 22 & & 20 \\
\hline Normalized projected emittance $(\mu \mathrm{m})$ & 0.35 & & $\approx 0.30$ \\
\hline Normalized core slice emittance $(\mu \mathrm{m})$ & 0.32 & & $\approx 0.20$ \\
\hline \multicolumn{4}{|l|}{ After compression: } \\
\hline Bunch length (rms) (ps) & 0.193 & & $0.3-0.6$ \\
\hline Peak current $(\mathrm{A})$ & 352 & & $120-240$ \\
\hline Normalized projected emittance $(\mu \mathrm{m})$ & 0.379 & & $\approx 0.40^{\mathrm{c}}$ \\
\hline Normalized core slice emittance $(\mu \mathrm{m})$ & 0.33 & & $\approx 0.20^{\mathrm{c}}$ \\
\hline
\end{tabular}

${ }^{\mathrm{a} U n c o m p r e s s e d ~ b e a m ~ o n l y . ~}$

b $85 \mathrm{MV} / \mathrm{m}$ for the CTF photocathode gun.

${ }^{\mathrm{c}}$ Measured for a peak current of $150 \mathrm{~A}$.

The main design aims leading to this choice of optics are (i) small $\beta$-functions $\left(\beta_{x, y}<50 \mathrm{~m}\right)$ throughout the lattice; (ii) a small horizontal $\beta$-function between the third and fourth dipole magnet of the bunch compression chicane to limit potential detrimental effects from coherent synchrotron radiation; (iii) adequate time resolution for longitudinal bunch measurements with the rf deflector at a profile monitor in the high-energy spectrometer; (iv) appropriate energy resolution at the high-energy spectrometer; (v) proper optics for the emittance measurement (in this case based on a single-quadrupole scan, see Sec. VI B for details).

TABLE II. Design parameters of the SwissFEL Injector Test Facility for the low-charge mode (10 pC bunch charge), as far as different from the high-bunch-charge mode, with comparison to typically realized values. (Systematic compression studies have only been carried out for the high-charge case.)

\begin{tabular}{lcc}
\hline \hline Parameter & Design & Realized \\
\hline Intrinsic emittance contribution $(\mu \mathrm{m})$ & 0.072 & $\approx 0.04$ \\
Bunch length $(\mathrm{rms})(\mathrm{ps})$ & 1.05 & 0.7 \\
Peak current $(\mathrm{A})$ & 3.0 & 4.0 \\
Normalized projected emittance $(\mu \mathrm{m})$ & 0.095 & $\approx 0.15$ \\
Normalized core slice emittance $(\mu \mathrm{m})$ & 0.078 & $\approx 0.10$ \\
Gun laser parameters & & \\
Spot diameter on cathode $(\mu \mathrm{m})$ & 300 & \\
Pulse length (FWHM) $(\mathrm{ps})$ & 3.7 & 2.5 \\
\hline \hline
\end{tabular}

The SITF was designed for two standard bunch charge modes, a high-charge mode with $200 \mathrm{pC}$ bunch charge, and a low-charge mode with $10 \mathrm{pC}$, in accordance with the planned SwissFEL bunch charge range of 10-200 pC. Table I summarizes the main design beam parameters for the high-charge mode and compares them to typically achieved values. The corresponding numbers for the lowcharge mode, where different from the high-charge mode, are listed in Table II. Note that systematic measurements with compressed beams have only been done for the highcharge mode.

The SITF typically operated at a single-bunch repetition rate of $10 \mathrm{~Hz}$. In contrast SwissFEL will operate at a repetition rate of $100 \mathrm{~Hz}$. The SITF was deliberately designed to run at $10 \mathrm{~Hz}$, which allowed the demonstration of the required beam parameters at somewhat relaxed requirements on hardware and shielding components. Moreover, the overall cooling layout of the CTF rf gun did not allow for repetition rates higher than $10 \mathrm{~Hz}$. The SwissFEL gun by contrast is designed to run at up to $400 \mathrm{~Hz}$ and was tested at a rate of $100 \mathrm{~Hz}$ at the SITF, albeit as an isolated system only. Several of the other rf stations were also operated individually at $100 \mathrm{~Hz}$ during dedicated test runs without beam.

\section{OPERATION OVERVIEW}

In this section we give a brief overview of the four main operation phases of the SITF, dating from March 2010 until 
October 2014. Driven by the progressive evolution of the machine, each operating phase had its own primary focus.

\section{A. Phase I: Gun commissioning}

The first phase of commissioning, Phase I, was dedicated to the commissioning of the rf gun and the low-energy beam diagnostics [31]. It started immediately after the completion of the component installation in the gun section, with first photoelectrons generated on 12 March 2010. The booster linac was still undergoing installation during this phase and was separated from the gun area by a concrete shielding wall. For this initial measuring period, the first girder of the S-band booster was replaced by a temporary diagnostics girder with additional profile monitors for direct measurements at the future booster entrance. After the removal of the shielding wall at the end of May 2010, gun commissioning continued for another month, during which beam operation was limited to evening and night hours due to the ongoing installation of the booster hardware during working days.

\section{B. Phase II: Basic booster commissioning}

For Phase II the entire beam line down to the beam dump was available. In place of the bunch compressor, which was still under construction at the time, a simple tube was installed to connect the booster to the diagnostics line downstream. On the booster side, only two out of the four structures were available for acceleration, thus limiting the final beam energy to about $165 \mathrm{MeV}$ in this commissioning phase. For most measurements the first structure was operated at a reduced gradient to fulfill the optimal conditions for emittance compensation, giving a final beam energy of about $130 \mathrm{MeV}$. Phase II started in early August 2010, focusing first on preparing the machine for an official inauguration ceremony held on the 24th of the month. At this occasion the beam was successfully accelerated to $165 \mathrm{MeV}$ for demonstration purposes, but many systems were still incomplete. Therefore a further installation period of several weeks followed immediately after the inauguration. Beam operation under limited conditions recommenced in midOctober, alternating with more installation work on a weekly basis. The initial set of installation tasks was completed by the end of the year, such that starting from early 2011, the injector was fully available for beam development work. The first weeks of 2011 were dedicated to setting up (matching) and verifying the beam optics for various configurations and to first optimizations of the transverse beam emittance. In mid-March, the transverse deflecting cavity was put into operation for the first time, allowing the first bunch length measurements. Phase II commissioning lasted until the end of May 2011. For most of the beam development work in Phases I and II, the robust and reliable Nd:YLF laser system was used for the generation of the electrons.
The considerably more complex Ti:sapphire laser system was only used towards the end of Phase II (see Sec. IVA).

\section{Phase III: Bunch compression and phase-space linearization}

During the summer of 2011, the movable magnetic chicane intended for bunch compression was installed between the booster and the diagnostics sections of the injector beam line. The following beam commissioning period, Phase III, in principle started in September of the same year, but suffered considerably from several problems with the rf systems, which severely hampered further beam development. As a consequence, the following months were mainly dedicated to the consolidation of the $\mathrm{rf}$ systems, with limited beam operation making use of the gun and the third booster structure only. During another shutdown in February 2012, the repaired fourth booster structure was reintegrated. The same shutdown was used to add the requisite infrastructure for the $\mathrm{THz}$ diagnostics system at the bunch compressor exit.

By April 2012, the rf repair work had been completed and the injector could be run with all accelerating structures for the first time. With the refurbished rf system in operation, it did not take long to achieve several important milestones within the same month: the design energy of $250 \mathrm{MeV}$ was attained, the first slice emittance measurements were performed and bunch compression was demonstrated for the first time. Since the X-band cavity had not yet been installed, only nonlinear compression could be realized at this time, with a bunch length reduction from $3.6 \mathrm{ps}$ to $0.2 \mathrm{ps}$ (rms). The further study of the compressed beam was postponed to the time when the linearizing cavity would be available.

In the period from May to July 2012 the focus was on the systematic optimization of the uncompressed beam, which resulted in emittance values well below the SwissFEL design figures (see Sec. VII B). In this context, a number of beam characterization methods were developed or refined, as will be described in detail in Sec. VI. The X-band cavity was installed for the first time in another shutdown in August, but had to be removed again due to a design flaw of the mover system. In the time after the summer shutdown the injector test facility was used for systematic measurements of the intrinsic emittance of copper cathodes and for the testing and development of various diagnostics systems, in particular charge monitors and bunch arrival-time monitors. These and similar activities based on uncompressed beam were resumed after the successful reintegration of the X-band cavity in January 2013 (with a redimensioned mover system), since the cavity could not yet be powered due to delays with the preamplifier system. During the same shutdown in January, the rf gun was equipped with a load-lock chamber to facilitate the exchange of cathodes. 
In June 2013 the X-band cavity was finally ready for beam operation and the experimental investigation of linearly compressed bunches could commence. The initial measurements revealed a few surprises, such as a growth in slice emittance and a fragmentation of the longitudinal phase space with progressive compression, and triggered a series of systematic investigations of these phenomena (see Sec. VII C). The compressed bunches were also used to test concepts of bunching monitors based on $\mathrm{THz}$ radiation emitted by the shortened bunches in the last dipole of the magnetic chicane.

Toward the end of Phase III, a modification of the $\mathrm{Nd}$ :YLF laser opened up the possibility to accelerate two electron bunches in the same rf pulse, separated by $28 \mathrm{~ns}$. These two-bunch tests were carried out in the context of diagnostics developments in view of the final configuration of SwissFEL, where two undulator lines will be fed simultaneously at $100 \mathrm{~Hz}$. In the last week of Phase III operations, at the end of October 2013, a copper cathode coated with cesium telluride $\left(\mathrm{Cs}_{2} \mathrm{Te}\right)$ was successfully tested. The significantly higher quantum efficiency allowed checks of diagnostics components at bunch charges exceeding $1 \mathrm{nC}$, but at a reduced beam energy to keep the beam power within limits.

In the course of Phase III operations, several campaigns to measure ambient radiation levels were carried out to evaluate shielding and instrumentation options for SwissFEL [32,33].

\section{Phase IV: Undulator experiment and new SwissFEL gun}

The fourth and last commissioning phase (Phase IV) was mainly dedicated to the real-conditions test of two crucial hardware components for SwissFEL: an undulator prototype and the new SwissFEL electron gun.

The installation of the undulator prototype in the diagnostics section of the injector (at the location of the former FODO channel) was carried out in the last weeks of 2013. Recommissioning of the machine started on January 14, 2014, still using the CTF rf gun for the generation of electrons. After a swift commissioning sequence, ultraviolet FEL radiation from the undulator exhibiting SASE characteristics was observed within two days (see Sec. VIII for details). The distorted photon beam profile measured at the undulator exit, however, hinted at a photon beam alignment problem in this vicinity, which prevented a detailed characterization of the radiation. In addition, a failure of the klystron driving the last two S-band structures soon compromised booster performance. A brief shutdown was consequently scheduled to rearrange the beam line after the undulator and to repair the defective klystron. While the first effort was successful the repair of the klystron took longer than initially expected. For the remainder of the undulator experiment, the beam energy was therefore limited to a maximum of about $130 \mathrm{MeV}$, thereby shifting the FEL radiation into the optical range. After some basic FEL characterization studies, the undulator setup was mainly used for testing alignment schemes and to measure the beam kicks at the undulator entrance and exit. These undulator measurements were alternating with further diagnostics developments and more intrinsic emittance measurements, in particular a systematic comparison between pure copper and cesium-telluride coated cathodes.

To spare the undulator prototype unnecessary radiation exposure in the test facility, it was again removed during the following shutdown in April-May 2014. At the same occasion, the CTF gun No. V was replaced by the newly manufactured SwissFEL rf gun, capable of running at $100 \mathrm{~Hz}$ repetition rate. After a brief conditioning period it reached its nominal power without difficulty.

The first characterization of the new gun under operating conditions, at repetition rates of both 10 and $100 \mathrm{~Hz}$, yielded very satisfying results, among them a significant reduction in dark current compared to the previously installed gun, as described in Sec. IV C 1 . The tests at $100 \mathrm{~Hz}$ were performed in stand-alone mode, since the injector as a whole was not designed to run at that rate, as stated earlier.

The remaining beam dynamics and diagnostics measurements could once again profit from the full beam energy, as the klystron driving the two last booster structures had been replaced by a new one. Measurements were, however, impeded by the limited bunch charge provided by the installed copper cathode, which exhibited a low quantum efficiency. The problem was exacerbated by the fact that the cathode could no longer be easily exchanged owing to a design flaw in the load-lock chamber, which only became evident with the new rf gun. Another brief shutdown in mid-July 2014 was used to apply a temporary fix to the load-lock chamber in addition to the exchange of the bunch compressor dipole magnets (see Sec. IVE) and the installation or replacement of various diagnostics components. Unfortunately, the regained ability to exchange cathodes only revealed that all remaining copper cathodes exhibited quickly deteriorating quantum efficiencies, leveling at rather low values. To have access to the full range of bunch charges, the remaining program was therefore carried out with cesiumtelluride-coated cathodes. The primary goal of the last weeks of operation was the investigation and possible mitigation of the emittance growth in conjunction with compression (Sec. VII C). These beam dynamics measurements were again performed in alternation with diagnostics developments and some final intrinsic emittance studies.

Injector operations came to an end in the early hours of October 13, 2014.

\section{SUBSYSTEMS AND OPERATIONAL EXPERIENCE}

We describe the main technical subsystems constituting the SITF and summarize the experience gained during the 
TABLE III. SwissFEL gun laser requirements and achieved performance of the Ti:sapphire and Nd:YLF amplifier systems used at the SITF.

\begin{tabular}{|c|c|c|c|c|}
\hline Parameter & Unit & $\begin{array}{l}\text { Requirement } \\
\text { (SwissFEL) }\end{array}$ & Ti:sapphire & Nd:YLF \\
\hline Pulse duration & ps & $3-10$ & $3-10$ & 8 \\
\hline Pulse rise-time & ps & $<0.7$ & $0.5-1$ & 5 \\
\hline Temporal pulse shape & & Flat-top & $\begin{array}{l}\text { Flat-top-like, stacked, } \\
\approx 10 \% \text { modulation }\end{array}$ & Gaussian \\
\hline Pulse energy at cathode & $\mu \mathrm{J}$ & up to 30 & $<40$ & $<20$ \\
\hline Central wavelength & $\mathrm{nm}$ & $250-300$ & $257-280$ & 262 \\
\hline UV energy stability at cathode & $\%(\mathrm{rms})$ & $<0.5$ & $1-2$ & $0.9-1.2$ \\
\hline Transverse profile & & Uniform & $\begin{array}{l}\text { Truncated Gaussian, } \\
\approx 10 \% \text { modulation }\end{array}$ & $\begin{array}{l}\text { Truncated Gaussian, } \\
\approx 10 \% \text { modulation }\end{array}$ \\
\hline Laser-to-rf jitter & fs & $<100$ & $\begin{array}{l}<30 \text { at oscillator, } \\
\approx 160 \text { at cathode }\end{array}$ & $\begin{array}{l}<40 \text { at oscillator, } \\
\approx 130 \text { at cathode }\end{array}$ \\
\hline $\begin{array}{l}\text { Beam pointing stability at } \\
\text { cathode (relative to laser beam size) }\end{array}$ & $\begin{array}{l}\% \text { (peak- } \\
\text { to-peak) }\end{array}$ & $<1$ & 0.15 & 0.15 \\
\hline
\end{tabular}

time of operation of the test facility. Where applicable, lessons learnt for the design and construction of SwissFEL are presented and elaborated on.

Apart from the bunch compressor dipole magnets, which are discussed in Sec. IV E, we will not further describe the various magnets used for beam manipulation at the SITF, since in most cases standard electromagnet solutions have been chosen $[34,35]$.

\section{A. Laser system}

Two different laser systems based on Ti:sapphire and $\mathrm{Nd}$ : YLF laser technology were employed for operating the photo-electron guns at the SITF. Both the Ti: sapphire and the Nd:YLF systems start with oscillators running at 83.275 $\mathrm{MHz}$ and actively synchronized within less than $50 \mathrm{fs}$ [36] to an external radio-frequency master clock running at $1498.956 \mathrm{MHz}$ (the 7th harmonic of the master clock, see Sec. IV H). They act as seed for the corresponding amplifier stages delivering $\mathrm{mJ}$ pulses at a repetition rate of up to $100 \mathrm{~Hz}$ (10 Hz for normal injector operation). The requirements and performance for the SwissFEL gun laser are listed in Table III.

In the following we give a short description of the two laser systems and their performance for pulse shaping. In the Nd:YLF laser system (Time-Bandwidth Products, Jaguar) the oscillator is followed by a single regenerative amplifier stage. It delivers transform-limited $10 \mathrm{ps}$ long pulses at a wavelength of $1047 \mathrm{~nm}$ with up to $2 \mathrm{~mJ}$ pulse energy. These pulses are frequency quadrupled in two subsequent collinear type-I second harmonic generators. For second-harmonic generation an antireflection (AR) coated BBO (beta barium borate) crystal $\left(\theta=23^{\circ}\right.$, $\mathrm{AR} / \mathrm{AR}$ at $1048 \mathrm{~nm} / 1048 \mathrm{~nm}+524 \mathrm{~nm}, \quad d=4 \mathrm{~mm})$ and a $2 \mathrm{~mm}$ thick uncoated BBO crystal $\left(\theta=44^{\circ}\right)$ are used. The resulting $8 \mathrm{ps}$ long, Gaussian-like pulses at $262 \mathrm{~nm}$ show a high energy stability $(0.3 \% \mathrm{rms}$ at $260 \mathrm{~nm}$ over a 10 minute period) thanks to the direct diode pumping of the Nd:YLF crystal. Figure 3 shows a cross-correlation measurement of the Gaussian UV longitudinal profile. The system can be operated either in a single-pulse or a doublepulse mode. For the latter a pulse replica is created in the UV using polarizing beam splitters. The amplitude and the delay of the two pulses can be adjusted to produce electrons in two buckets of the same rf pulse $28 \mathrm{~ns}$ apart.

The Ti:sapphire amplifier system (Amplitude Technologies, Pulsar) is based on broadband chirped-pulse amplification. The laser is equipped with advanced pulse shaping technology based on acousto-optic programmable modulators (Fastlite, Dazzler and Mazzler) that allows the production of broadband $(\Delta \lambda=100 \mathrm{~nm})$ or alternatively narrow-band pulses tunable within a range of 770-830 nm [37]. To achieve the required pulse stability six pump lasers (Quantel Laser, Centurion) are multiplexed in the final amplifier stage. This results in an improved pulse stability of $0.4 \% \mathrm{rms}$ in the near IR, which is a factor of $\sqrt{6} \approx 2.5$

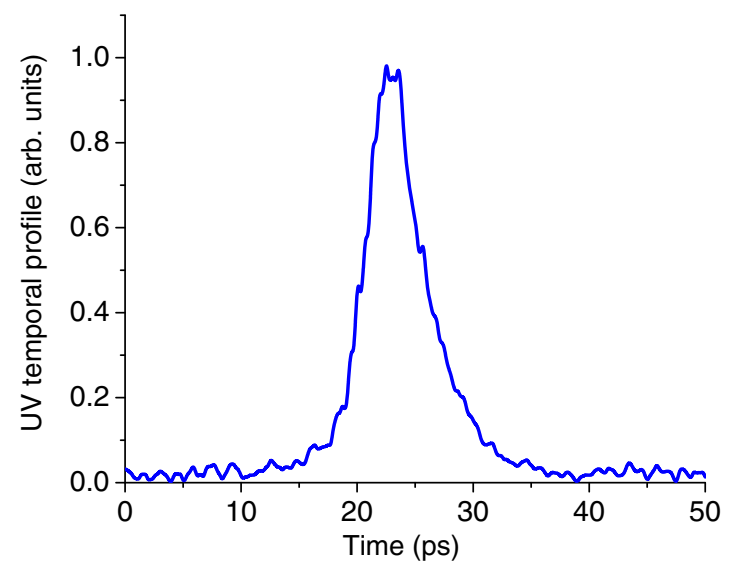

FIG. 3. Longitudinal pulse profile of the Nd:YLF laser, measured with cross-correlation in the UV. 


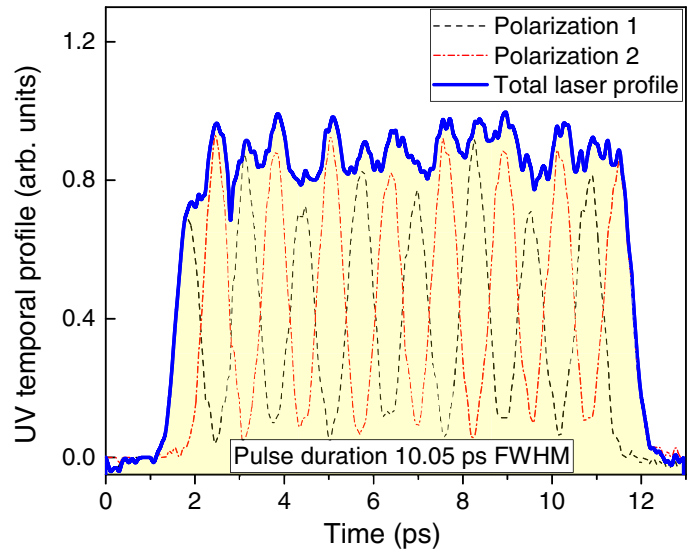

FIG. 4. Longitudinal pulse profile of the Ti:sapphire laser, measured with cross-correlation in the UV. The profile is obtained by pulse stacking with four crystals, resulting in 16 pulse replicas. The cross-correlations for s- and p-polarized pulses are done in sequence (black and red curves) and summed up numerically (blue curve)

better compared to a single pump laser (but entails higher maintenance cost). The near-IR output is frequency converted into the UV range by collinearly mixing the fundamental and second harmonic pulses in a type-I BBO $\left(\theta=44.3^{\circ}, \phi=0^{\circ}, d=0.5 \mathrm{~mm}, \mathrm{AR} / \mathrm{AR}\right.$ coated $)$. The 50 fs short UV pulses with an energy stability of $1.1 \%$ rms are then stretched to $500 \mathrm{fs}$ by employing a $10 \mathrm{~cm}$ long $\mathrm{CaF}_{2}$ block after temporal pulse shaping. Among the various explored temporal shaping schemes including UV Dazzler [38], chirp mixed UV generation [39] and 4-f pulse shaping, the technique of pulse stacking turned out to be the most efficient to produce flattop-like pulses in the picosecond range. Three to five $(n)$ birefringent crystals of increasing thickness $\left(L=2^{(n-1)} \times 400 \mu \mathrm{m}\right.$, $n=1, \ldots, 5)$ are used to produce $2^{n}$ replicas equally delayed in time and forming the 3 to $10 \mathrm{ps}$ long UV pulse (Fig. 4). The inherent amplitude modulations along the temporal pulse shape could be reduced to less than $20 \%$ (peak-to-peak).

A homogeneous transverse laser beam profile on the cathode is essential for the production of a low-emittance electron beam. Figure 5 schematically illustrates the beam transport and imaging sequence for the gun lasers in use at the SITF. The UV transverse beam profile is cleared from high-frequency spatial components by means of an inhouse designed biconical glass capillary acting as spatial Fourier filter. The filter is located in air for the Nd:YLF system and in vacuum for the Ti: sapphire system. Prior to beam transport the Gaussian-like UV beam intensity profile is truncated by a hard aperture of variable diameter. This approach has turned out to be more robust than other explored shaping schemes such as microlens arrays or the aspheric beam shaper. Beam transportation toward the cathode is performed in a high-vacuum transfer line $\left(10^{-7}\right.$ mbar) sealed with UV-transparent UV-fused silica windows (AR coated at $260 \mathrm{~nm}$ ). A single imaging lens projects the truncated profile onto the copper cathode in the rf gun with a demagnification ratio of $2.5: 1$.

The imaging lens is mounted on a motorized stage and allows adjustment depending on the wavelength chosen for operation. Part of the beam is reflected off from an AR

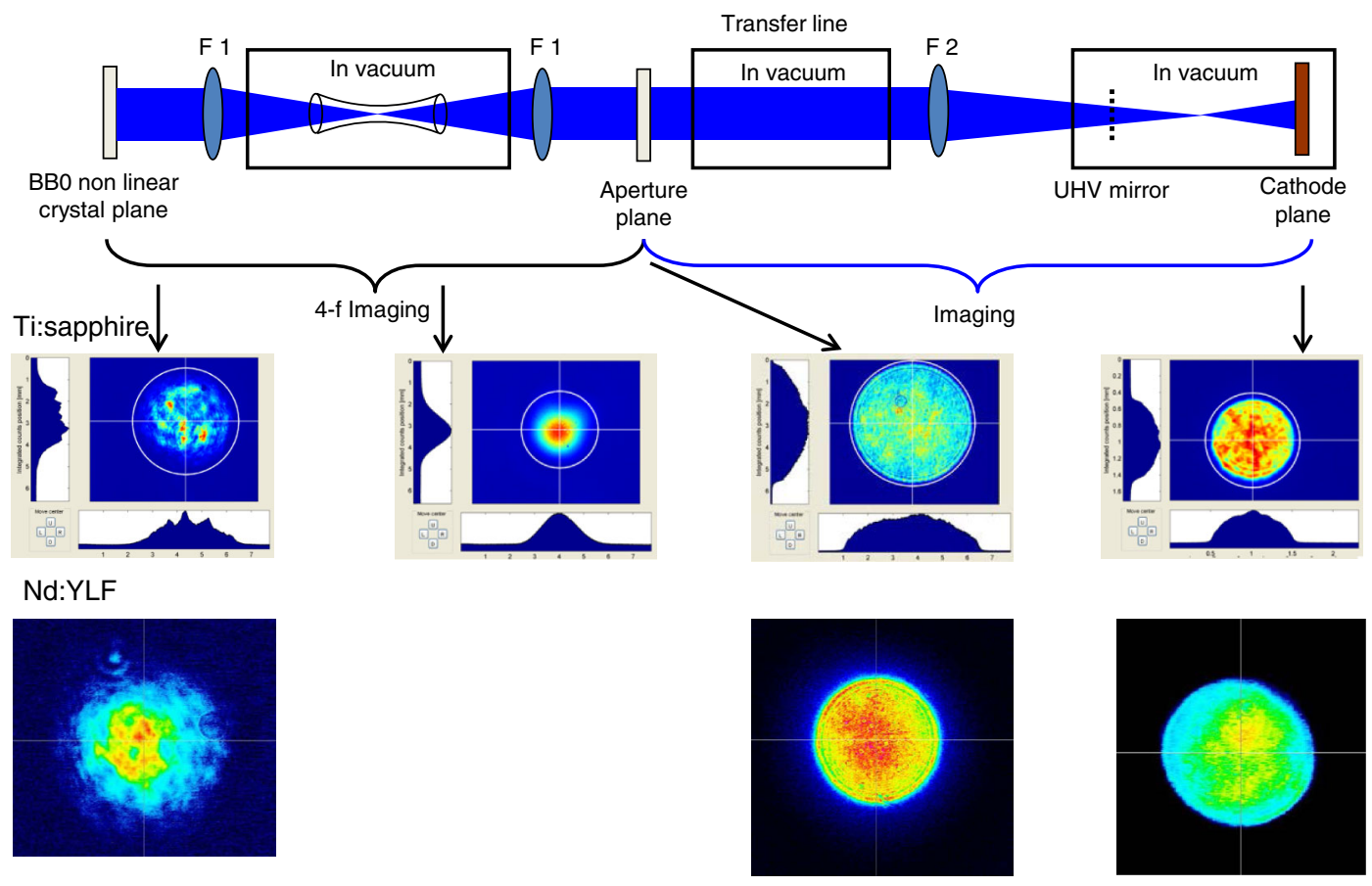

FIG. 5. Beam transport and transverse beam profiles along the beam line for the Ti:sapphire and Nd:YLF gun laser systems. 
coated wedge before entering the electron beam line. The first reflection is sent onto a K6 UV scintillator (Metrolux), placed at a plane at a distance equivalent to that to the cathode, acting as a virtual cathode. The visible light is then imaged onto a camera. The image is used to monitor beam profile, position, and size. A double-mirror piezo system in conjunction with the image analysis allows for pointing feedback stabilization. The second reflection from the wedge is used to monitor the laser energy (LaserProbe, RjP-465). Motorized stages allow inserting pyroelectric detectors (Gentec, QE25) into the direct beam for crosscalibration. A He-Ne laser is copropagated with the UV beam as a reference for alignment onto the cathode. Frequently used diagnostics are integrated into the injector control system and remotely accessible to operators. This includes a remotely controlled cross-correlation of the stacked pulse, online diagnostic of the UV pulse energy, the virtual beam profile at the cathode position and beam pointing.

For SwissFEL a different technology based on a

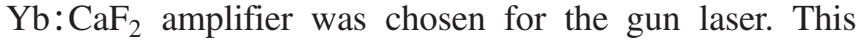
selection was motivated by the fact that it combines many advantages of both laser systems described above. It provides, for example, pulses with a high energy stability (due to direct pumping) and directly emits transformlimited $500 \mathrm{fs}$ pulses, which can be used for pulse stacking without need for additional dispersion. Furthermore, it features a significantly reduced complexity compared to a Ti:sapphire laser, with favorable consequences for system uptime and maintenance expenses.

\section{B. Photocathodes}

The backplanes of both the CTF and the SwissFEL gun feature circular openings where cathode plugs can be inserted. The cathode plugs are based on earlier designs developed at CERN for the CLIC test facility (see, e.g., Refs. [40,41]). An array of rf spring contacts arranged circularly around the cathode surface ensures electrical contact to the gun body (see Fig. 6). Thanks to a load-lock
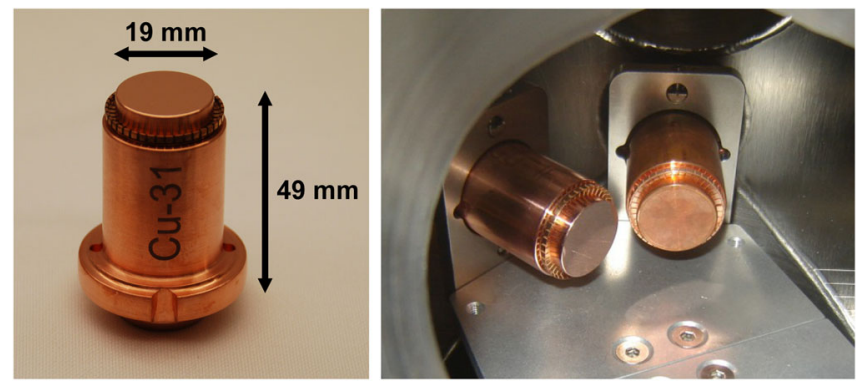

FIG. 6. SwissFEL gun cathode plug (copper, no coating), with dimensions indicated (left). Two cathode plugs mounted in the carousel holder of the load-lock chamber (right, see also Fig. 7). The rf contacts can be recognized around the circular cathode surfaces.

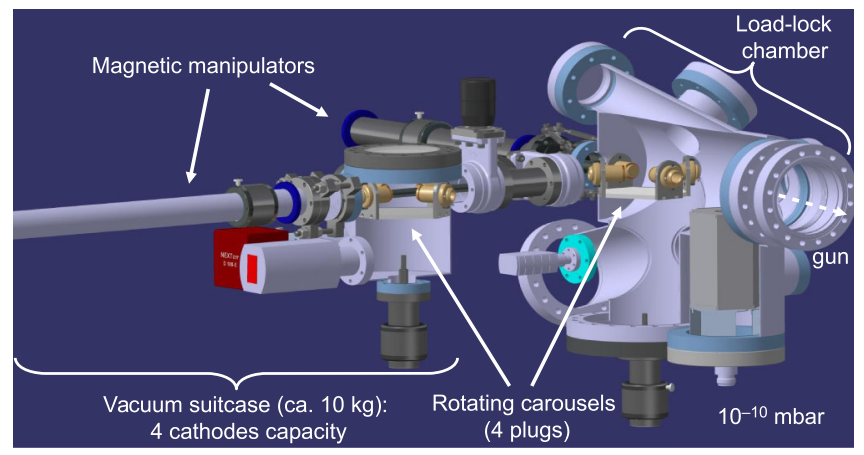

FIG. 7. 3D drawing of the vacuum suitcase connected to the load-lock chamber showing the cathode transfer principle and the two storage carousels.

system and a vacuum suitcase (Fig. 7 and Ref. [42]), cathodes can be cleaned and, if desired, coated with cesium telluride $\left(\mathrm{Cs}_{2} \mathrm{Te}\right)$ in the lab and then loaded behind the gun while staying under ultra-high vacuum (less than $10^{-9}$ mbar pressure). The load-lock chamber facilitates the cathode exchange considerably since no venting of the gun is necessary and, more importantly, it enables the use of semiconductor cathodes, which cannot be transported through air. The exchange of a cathode then only takes about half a day, including the rf conditioning of the new cathode. Up to four cathodes can be stored under vacuum in the load-lock chamber.

The fundamental parameters of a cathode are the local work function and the electric field enhancement parameter (see also Sec. VII A). These two parameters are strongly related to the material type, the surface roughness, and the surface contamination. Surface contaminants can be accumulated both during cathode preparation in air, as well as during operation in the gun, so that the initial QE can easily vary by a factor 10 from cathode to cathode (see Fig. 8). The QE also fluctuates with time because of the contaminants' activity on the surface (migration, bombardment,

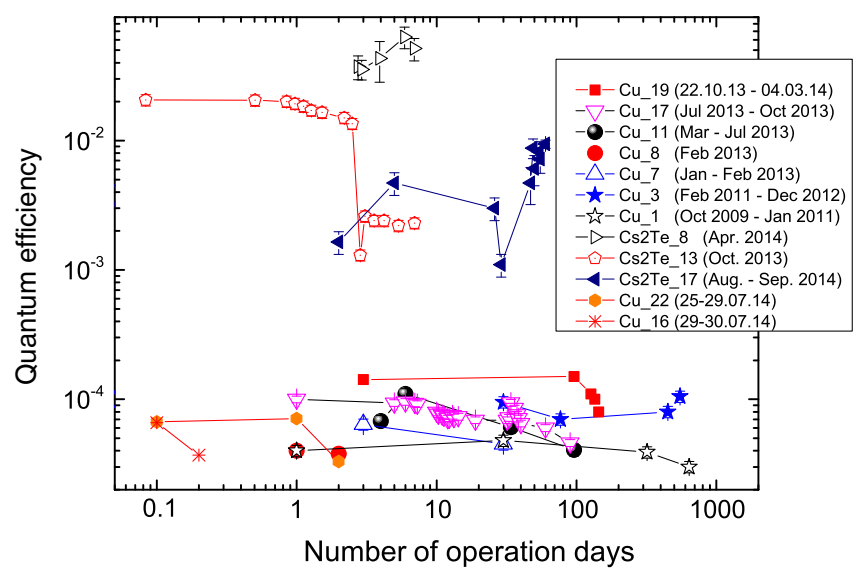

FIG. 8. Quantum efficiency evolution of the cathodes used at the SITF between October 2009 and September 2014. 
desorption etc.). To improve the QE stability and reproducibility of copper cathodes, we have established a preparation procedure [42], the most important step of which is the annealing procedure at $250^{\circ} \mathrm{C}$ over 10 hours to desorb the most detrimental contaminants like water. This procedure was implemented together with the load-lock installation in July 2013 and ensured initial QEs above $10^{-4}$ for newly installed copper cathodes.

The same treatment of the copper surface was applied for the preparation of cesium telluride cathodes, before evaporating $15 \mathrm{~nm}$ of Te and $25 \mathrm{~nm}$ of Cs onto the surface [43]. Indeed the load-lock chamber offers the possibility to compare copper and cesium telluride cathodes under the same gun and laser conditions, whereby QE and intrinsic emittance are the most relevant parameters to assess cathode quality. A high QE gives more laser energy reserve, which may be invested in laser shaping (emittance improvement), while a low intrinsic emittance directly increases the beam brightness. It is well known that cesium telluride provides a QE much higher than copper, and it is generally assumed that this advantage comes at the price of a higher intrinsic emittance. Measurements with the SwissFEL gun, described in more detail in Sec. VII A, showed a factor of 100 gain in QE at an increase in intrinsic emittance of only about 20\% [44]. What is more, the slower response time of cesium telluride relative to copper also helps to wash out the laser pulse time profile ripples in the case of flat-top laser pulses.

In conclusion, since cesium telluride exhibits the more favorable properties for long-term operation, if laser energy is limited, it has been selected as the baseline option for SwissFEL.

\section{Radio frequency systems}

Figure 9 shows a schematic layout of the injector $\mathrm{rf}$ system, featuring the S-band photocathode if gun (labeled FINSS), the S-band booster (FINSB), as well as the X-band linearizing system (FINXB) and the transverse deflector (F10D1) used for diagnostic purposes. Each rf plant consists of a high-voltage modulator and a klystron driving the connected accelerating structure (or structures). The S-band booster consists of four accelerating structures powered by three rf plants: the first two structures (FINSB01 and FINSB02), usually run at the on-crest

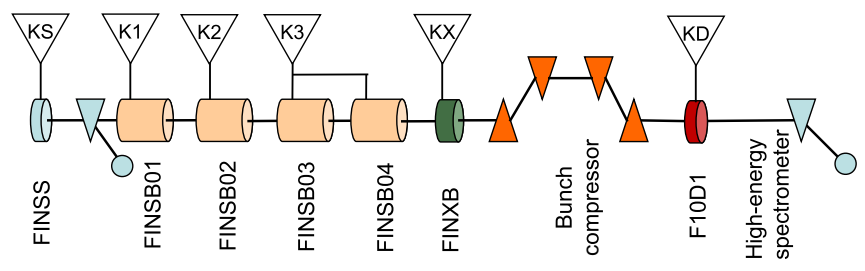

FIG. 9. Schematic layout of the SITF rf system, showing the accelerating structures as cylinders with the corresponding power sources (klystrons). phase, are driven by separate if plants each, whereas a single rf plant powers the last two structures (FINSB03 and FINSB04), which are operated off-crest to generate the energy chirp whenever the beam is to be compressed in the ensuing magnetic chicane.

In the following we describe each of the rf subsystems, placing particular emphasis on the photocathode if gun, for which a new design was developed and tested at the SITF.

\section{Photocathode rf gun}

The first commissioning phases of the SITF commissioning relied on the CTF gun No. V, a 2.6-cell gun originally developed for high-current, multi-bunch operation at the CLIC test facility [26,27]. The general geometry of this gun, as used in numerical simulations, is depicted in Fig. 10, along with electric field magnitudes in the horizontal and vertical planes. The unique feature compared to other designs is the large diameter of the first half cell, where the $\mathrm{TM}_{02}$ resonance is used as the main accelerating mode. This resonance is particularly well suited for compensating the beam loading associated with extremely high beam currents. At the modest currents required for SwissFEL, however, this feature is of no relevance.

The gun structure is rotationally symmetric except for the tuners, the pickups, and the coupling irises located in the last cell. The field asymmetries introduced in the accelerating mode have a diluting effect on the beam emittance, which is, however, well below the initial thermal emittance from the photocathode [45].

Operating at an rf power of $21 \mathrm{MW}$ and with a pulse length of typically $2 \mu \mathrm{s}$, the CTF gun provides an on-axis peak electric field of $100 \mathrm{MV} / \mathrm{m}$, with a maximum field of $85 \mathrm{MV} / \mathrm{m}$ at the cathode. The extracted electrons reach a nominal momentum of $7.1 \mathrm{MeV} / c$ at the gun exit. The repetition rate was generally kept at $10 \mathrm{~Hz}$ because the

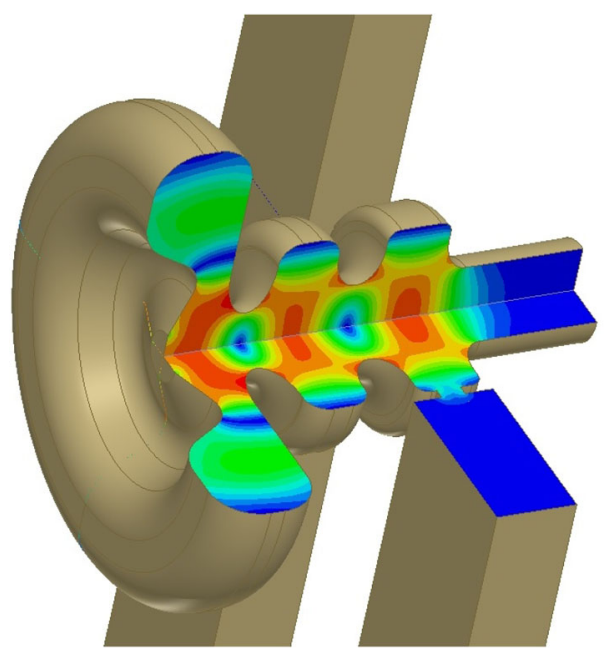

FIG. 10. Geometry of the CTF gun No. V, with magnitude of the electric field in the horizontal and vertical planes. 


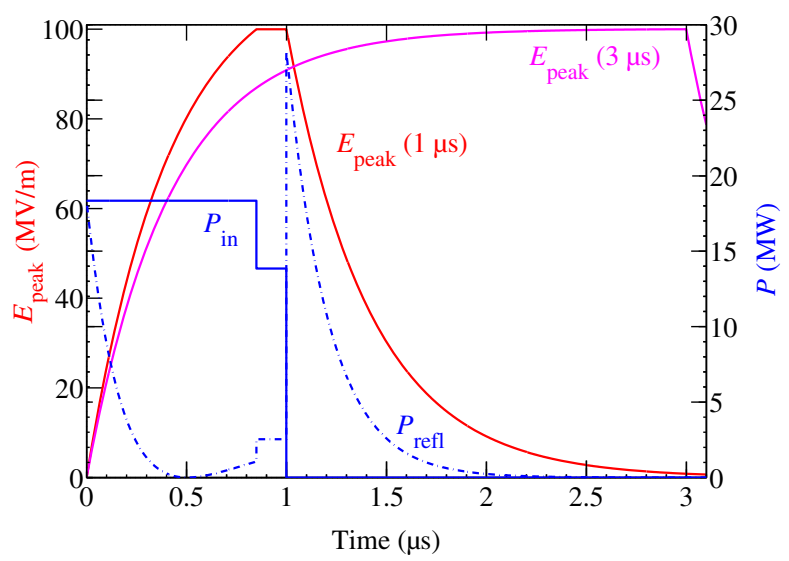

FIG. 11. Amplitude modulation scheme for the SwissFEL gun: input rf power (solid blue line), reflected power (dash-dotted blue line), and resulting peak gradient (red line) as a function of time for the nominal modulated rf pulse of $1 \mu \mathrm{s}$ total length. For comparison, the peak gradient for an unmodulated $3 \mu$ s pulse is also shown (magenta line) [28].

overall cooling channels are not compatible with operation at $100 \mathrm{~Hz}$. Indeed, for repetition rates higher than $20 \mathrm{~Hz}$ a significant overheating of the tuners was observed.

To meet the operational requirements of SwissFEL, in particular long lifetime and high reliability at a repetition rate of $100 \mathrm{~Hz}$, a new rf photocathode gun was designed and manufactured in house [28]. The geometry of the new SwissFEL gun is inspired by the 2.5-cell PHIN gun [46,47] and adopts some mechanical design aspects of the LCLS gun $[48,49]$. The SwissFEL gun design, shown in a 3D view in Fig. 12, is based on 2.6 cells operating with a nearperfect rotationally symmetric $\pi$-mode at S-band frequency, where each cell is equipped with a dedicated pickup. The middle cell is coupled to two rectangular waveguides, symmetrically arranged to cancel the dipolar component of the field. To reduce pulsed surface heating, the z-coupling scheme developed for the LCLS gun [49] is adopted. The racetrack interior shape of the coupling cell is optimized to keep the quadrupolar field components at a minimum [50].

Similar to the LCLS gun, our design opts for a large coupling coefficient $(\beta \approx 2)$ to decrease the filling time of the cavity. As a consequence, the rf pulse needed to reach the accelerating gradient can be shortened, resulting in a lower heat load and hence less mechanical stress on the gun body. The shorter rf pulse has the additional advantages of reducing both the amount of dark current originating from the gun and the probability of electric arcs. The length of the rf pulse can be further reduced through the application of a suitable amplitude modulation scheme of the rf input power [51]. In such a scheme, the input power level during the filling of the cavity is higher than that required to reach the desired accelerating gradient, but is then quickly reduced to a lower level once this gradient has been attained. The lower power level is sufficient to maintain

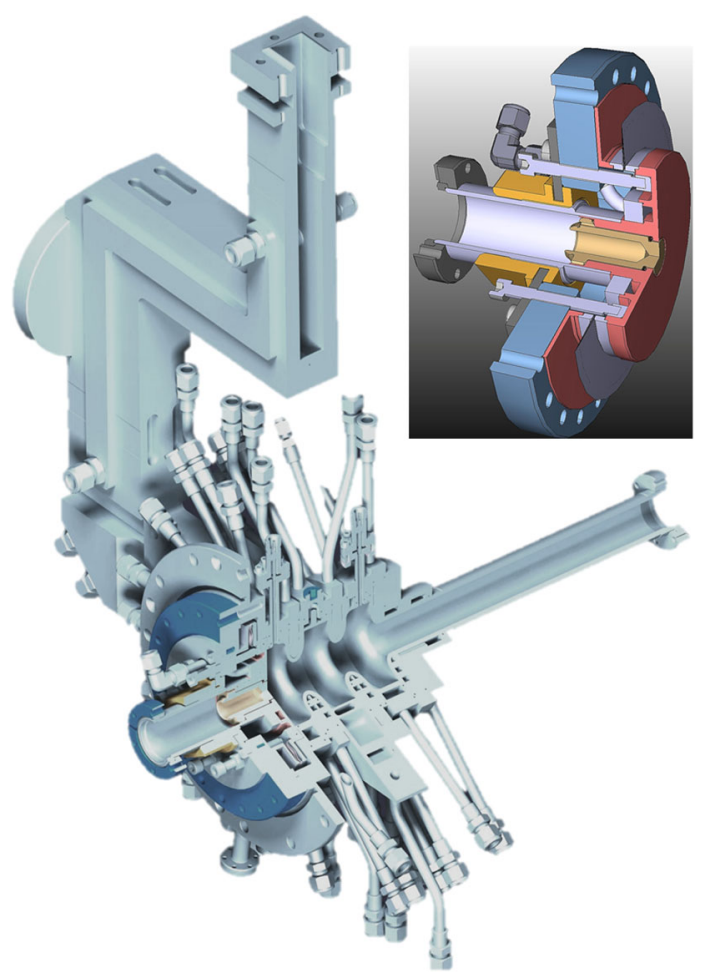

FIG. 12. Cutaway 3D views of the SwissFEL gun. Details of the backplane and the cathode plug are visible in the upper right figure.

the nominal accelerating gradient in the cavity and is provided for as long as the field is needed. In the case of the SwissFEL gun, the initial power level of about $18 \mathrm{MW}$ is maintained for $850 \mathrm{~ns}$ and then reduced to $14 \mathrm{MW}$ for the following $150 \mathrm{~ns}$, yielding a flat-top pulse compatible with the two-bunch operation with $28 \mathrm{~ns}$ bunch separation foreseen for SwissFEL (see Fig. 11).

Nominally, the gun provides an on-axis peak electric field of $100 \mathrm{MV} / \mathrm{m}$. In contrast to the CTF gun, the location of the on-axis peak field coincides with the cathode. Therefore the electrons, which are extracted at a similar laser injection phase, experience a higher gradient. The mean electron momentum at the gun exit is again 7.1 MeV/c as in the CTF setup.

The cooling layout of the SwissFEL gun allows operation at repetition rates up to $400 \mathrm{~Hz}$. At the nominal repetition rate of $100 \mathrm{~Hz}$ and a pulse duration of $1 \mu$ s the required input power is $18 \mathrm{MW}$ while the dissipated power averages to $900 \mathrm{~W}$.

The parts constituting the first SwissFEL gun were premachined and brazed at PSI [52], whereas the final machining, involving ultra-precision turning and milling, was performed at VDL Groep within the specified tolerances on the surface roughness of $R_{a} \leq 25 \mathrm{~nm}$. The finished gun was installed in the SITF in April 2014.

As described in Sec. IV B the gun features a circular opening in the backplane allowing the straightforward exchange of a cathode plug under vacuum through a 
TABLE IV. Simulated and measured SwissFEL gun rf parameters. The time constant refers to the rise time of the electromagnetic field inside the cavity.

\begin{tabular}{lcc}
\hline \hline Parameter & HFSS & Measurement \\
\hline$\pi$-mode frequency $(\mathrm{MHz})$ & 2997.912 & 2997.912 \\
$\beta$-coupling & 1.98 & 2.02 \\
Quality factor $Q_{0}$ & 13630 & $13690 \pm 100$ \\
Time constant $(\mathrm{ns})$ & 485 & 481 \\
Mode separation $(\mathrm{MHz})$ & 16.36 & 16.20 \\
Field balance $(\%)$ & $>98$ & $>98$ \\
Operating temperature $\left({ }^{\circ} \mathrm{C}\right)$ & 57.7 & 53.0 \\
\hline \hline
\end{tabular}

load-lock system. The upper right of Fig. 12 shows a cutaway view of the cathode plug inserted in the backplane.

Table IV lists the most important parameters of the gun, comparing measurement results to values obtained with $\mathrm{rf}$ simulations performed with the ANSYS High Frequency Structure Simulator (HFSS). Measurements at room temperature in the clean room showed that the resonant frequency depends on the force with which the cathode plug is pushed into the gun. Therefore the frequency spectrum and field balance were directly verified with the bead-pull method at the SITF before the start of rf conditioning and with the load-lock chamber installed. To measure the frequency and field balance as a function of the force applied to the cathode plug, the plug manipulator was equipped with a calibrated mechanical pressure gauge. Figure 13 (top) illustrates the results of the measurements for different forces. The rf gun is tuned at the nominal SITF S-band frequency of $2997.912 \mathrm{MHz}$ with a balanced field when a force of more than $600 \mathrm{~N}$ is applied at a working temperature of $53.0^{\circ}$. The lower part of Fig. 13 shows onaxis electric field profiles for different positions of the cathode plug as obtained from bead-pull measurements with a ceramic bead of $3 \mathrm{~mm}$ diameter. The cathode plug used for these measurements has a central hole to accommodate the bead-pull wire. At the nominal frequency the
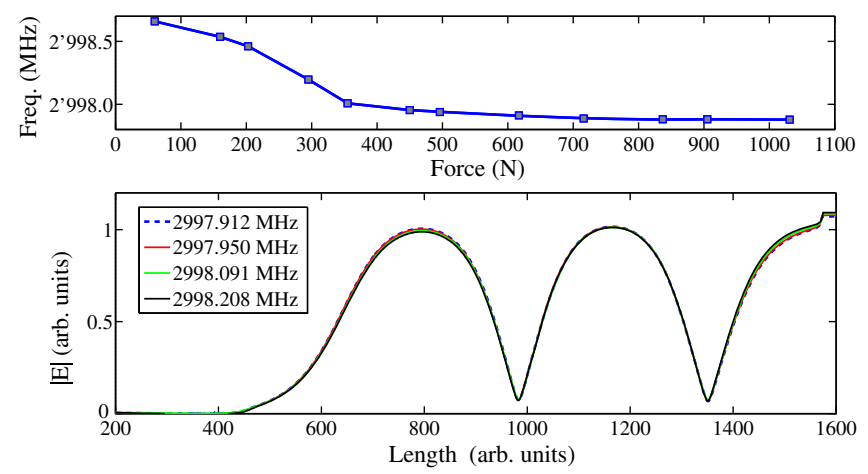

FIG. 13. Resonant frequency of the $\pi$-mode as a function of the force applied to the cathode plug (top). On-axis electric field profiles for different frequencies, obtained by varying the position of the cathode plug (bottom).

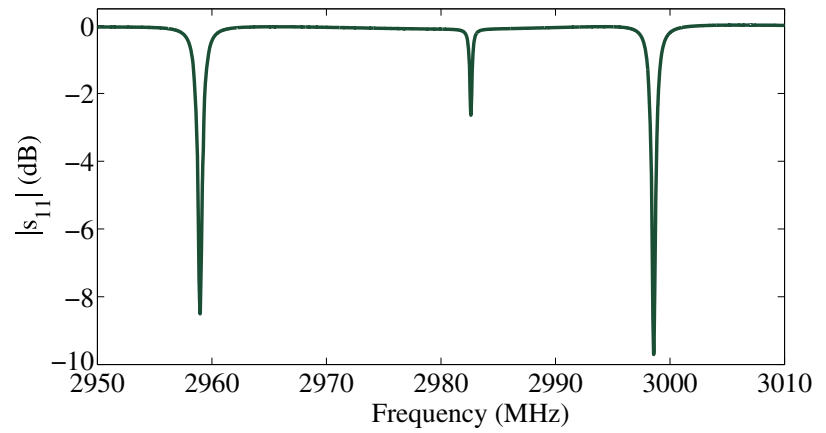

FIG. 14. Measured reflection coefficient $\left(\left|s_{11}\right|\right)$ as a function of frequency revealing the frequency spectrum of the SwissFEL gun. The operating frequency in vacuum is $2997.912 \mathrm{MHz}$ at $53^{\circ} \mathrm{C}$. The frequency separation to the adjacent mode is 16.2 $\mathrm{MHz}$.

field ratios (balances) are $E_{2} / E_{c}=1.02$ and $E_{1} / E_{c}=1.01$, where $E_{c}$ is the field on the cathode and $E_{2}$ and $E_{1}$ are the fields in the middle and last cell, respectively. The frequency spectrum at operational conditions is shown in Fig. 14. The frequency separation from the adjacent mode is $16.2 \mathrm{MHz}$, in excellent agreement with the simulation result.

The frequency and field balance measurements indicate that no additional tuning on the rf gun geometry is necessary, once the adequate force is applied on the cathode plug. Furthermore, the operating temperature is only $4.7^{\circ} \mathrm{C}$ away from the design value (Table IV), which corresponds to a difference in frequency of about $220 \mathrm{kHz}$. These results bear testimony to the excellent mechanical precision with which the gun parts were manufactured.

The SwissFEL gun was tested at full power in the SITF bunker using the rf distribution system of the injector equipped with a ScandiNova K2 solid-state modulator and a Thales TH 2100L (45 MW) $100 \mathrm{~Hz}$ S-band klystron. The klystron output is connected to the gun via a $5 \mathrm{~kW}$ averagepower (35 MW peak-power) rf circulator (AFT microwave). An active temperature control system (Regloplas 90S) stabilizes the gun body temperature. Typical curves illustrating the $\mathrm{rf}$ conditioning progress are shown in Fig. 15. The rf conditioning was performed at a pulse repetition rate of $100 \mathrm{~Hz}$, by gradually increasing the $\mathrm{rf}$ power with the help of an automatic conditioning tool while increasing the pulse width from 0.3 to $1.0 \mu \mathrm{s}$. After the conditioning period the vacuum level with rf power was measured to be around $10^{-10}$ mbar. The nominal rf power of $18 \mathrm{MW}$ for an rf pulse width of $1 \mu \mathrm{s}$ was already attained in the first week of operation.

The momentum and momentum spread of the electron beam can be measured with the low-energy spectrometer arm located after the rf gun and before the first travelingwave structure. To limit the contribution of the natural beam size to the dispersion observed in the spectrometer, the beam is focused horizontally by a small quadrupole magnet in front of the bending magnet. The rms energy 

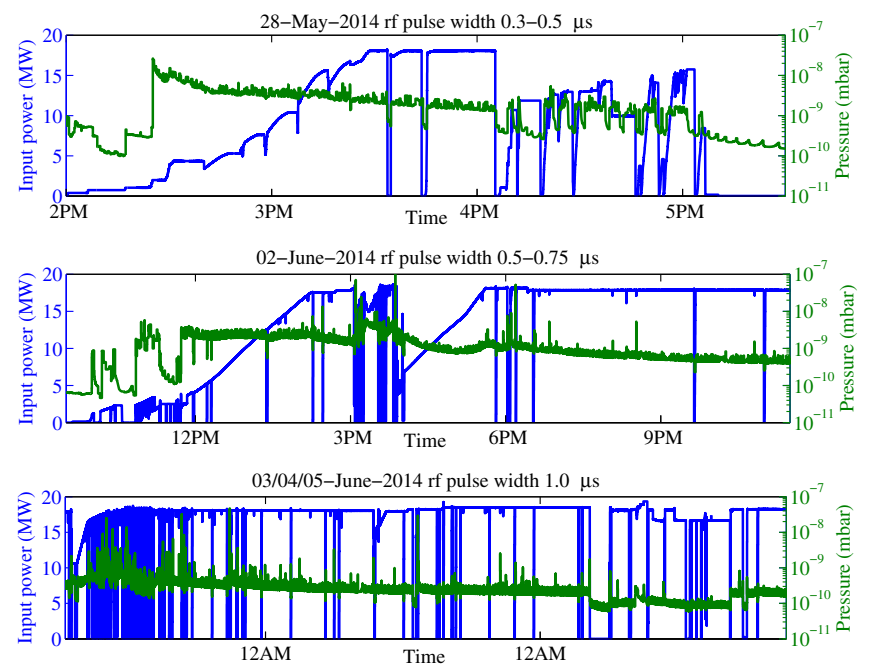

FIG. 15. Progress in rf conditioning the SwissFEL gun toward high power. The pressure gauge is mounted on the rf waveguide at the input of the rf gun.

resolution at the spectrometer is $4 \mathrm{keV}$ plus a systematic error arising from uncertainties of the dipole magnetic field of less than $0.1 \%$. Figure 16 shows an example of nonGaussian horizontal profile at the spectrometer YAG:Ce scintillating screen (Sec. IV F 3) with a modified superGaussian function (see Ref. [19] for details) fitted to it. The horizontal axis is converted to $\mathrm{MeV}$ according to the dipole dispersion of $0.387 \mathrm{~m}$. Figure 17 shows the jitters in bunch mean energy and energy spread at the gun exit determined from 49 nonconsecutive shots over $30 \mathrm{~s}$ with a laser pulse repetition rate of $10 \mathrm{~Hz}$ for rf running at $10 \mathrm{~Hz}$ and $100 \mathrm{~Hz}$. The rms relative mean energy jitters are $0.023 \%$ and $0.020 \%$ for $100 \mathrm{~Hz}$ and $10 \mathrm{~Hz}$, respectively. The rms jitter of the energy spread is $0.5 \mathrm{keV}$ for both repetition rates.

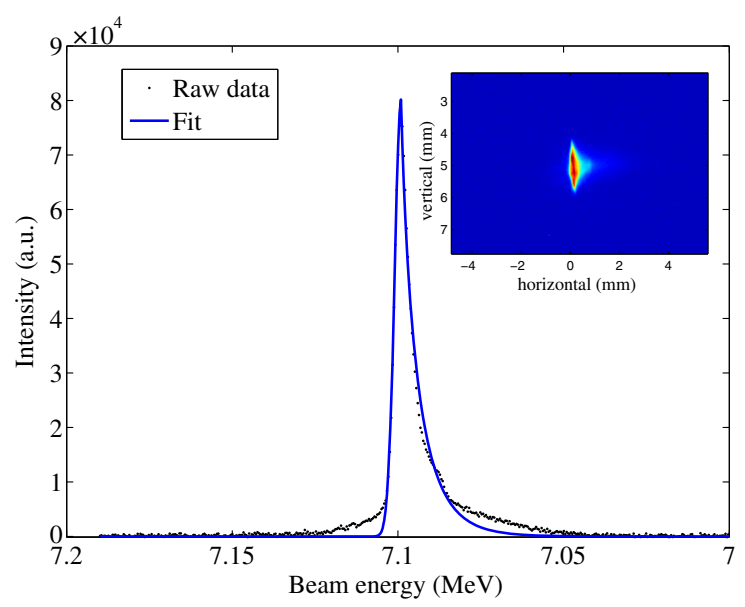

FIG. 16. Example of a non-Gaussian horizontal beam profile in the spectrometer at $7.1 \mathrm{MeV}$ (black points), with a modified super-Gaussian function fitted to the data (blue line). The inset shows the original screen image.
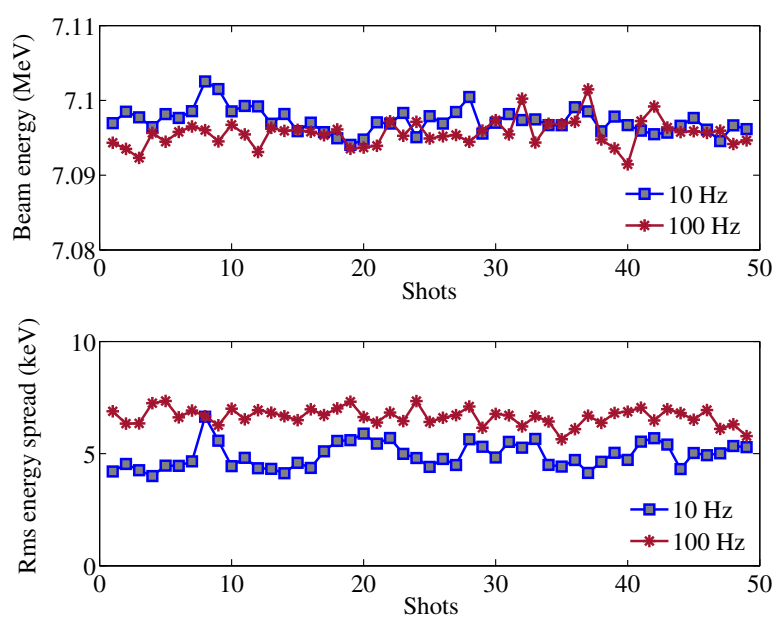

FIG. 17. Bunch mean energy jitter (top) and bunch rms energy spread (bottom) at the gun exit determined from 49 nonconsecutive shots over $30 \mathrm{~s}$ with a laser pulse repetition rate of $10 \mathrm{~Hz}$ for rf running at $10 \mathrm{~Hz}$ (blue squares) and $100 \mathrm{~Hz}$ (red asterisks).

During the high-power tests the emitted dark current was also measured, an important quantity in view of the longterm operation of SwissFEL. Earlier measurements performed on the CTF gun resulted in alarmingly high dark current values, triggering systematic studies on possible collimation strategies [53].

The average charge arising from the photocathode and the rf gun structure within one rf pulse was measured as a function of the peak cathode field for different pulse durations by use of an insertable coaxial Faraday cup positioned at the gun exit (see Sec. IV F 1). In these measurements, the gun solenoid is adjusted in each case to maximize charge collection of the Faraday cup. At nominal operating conditions with a peak electric field on the cathode of $100 \mathrm{MV} / \mathrm{m}$ and a total $\mathrm{rf}$ pulse length of $1 \mu \mathrm{s}$, the integrated dark current collected by the Faraday cup is approximately $50 \mathrm{pC}$ for the SwissFEL gun [54]. For comparison, the integrated dark current observed from the CTF gun amounts to about $1.4 \mathrm{nC}$ under nominal conditions $(85 \mathrm{MV} / \mathrm{m}$ on the cathode, $2 \mu \mathrm{s}$ rf pulse length) [53].

The dark charge was also imaged using a $200 \mu \mathrm{m}$ thick YAG: Ce scintillating screen placed in the same housing as the Faraday cup. Figure 18 shows a comparison between images of the dark charges observed in the CTF and SwissFEL guns. In both cases the electrons are focused with the gun solenoid on the same view screen.

The increase of the integrated dark current with the cathode field, as measured with the SwissFEL gun for a diamond-milled polycrystalline copper cathode, is plotted in Fig. 19 for repetition rates of $10 \mathrm{~Hz}$ and $100 \mathrm{~Hz}$. The emission of electrons in a period of the rf field is essentially generated by field emission, which is governed by the wellknown Fowler-Nordheim equation [55]. Using this equation with a copper work function of $4.65 \mathrm{eV}$, and taking into 

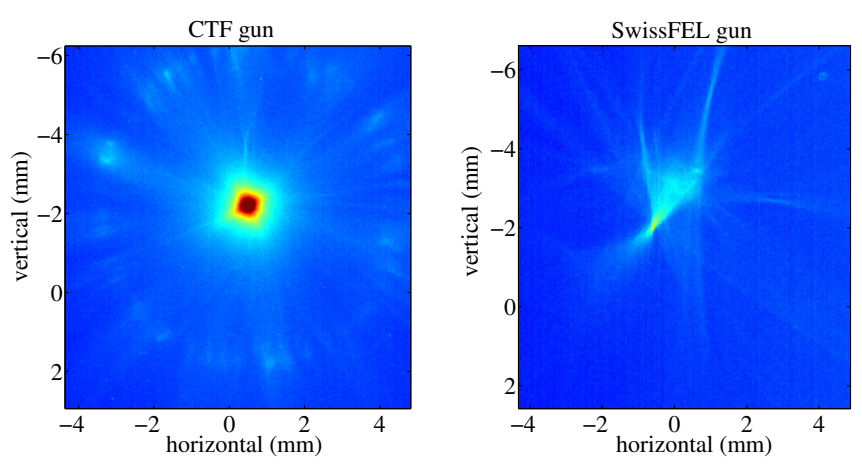

FIG. 18. Example images of dark current at nominal conditions on a YAG:Ce screen near the gun exit for the CTF (left) and SwissFEL (right) guns.

account the transient behavior due to the filling time (field rise time) of the gun, we estimate the field enhancement factor $\beta$ to lie in the range 63-68.

\section{S-band booster}

The S-band booster comprises four constant-gradient traveling-wave accelerating structures with $2 \pi / 3$ phase advance [56]. The $4.15 \mathrm{~m}$ long structures are separated by short drifts, in which corrector magnets, BPM and screen monitor are accommodated, and consist of 122 cells each: 120 regular cells and two coupler cells. The latter are of the double-feed type with a racetrack cross section to cancel dipolar and minimize quadrupolar field components at lower beam energies. The structures were designed at PSI and manufactured at RI Research Instruments. Table V summarizes a few important parameters of the S-band structure design.

The four booster structures are driven by three rf plants (see Fig. 9), each consisting of a ScandiNova K2 solid-state

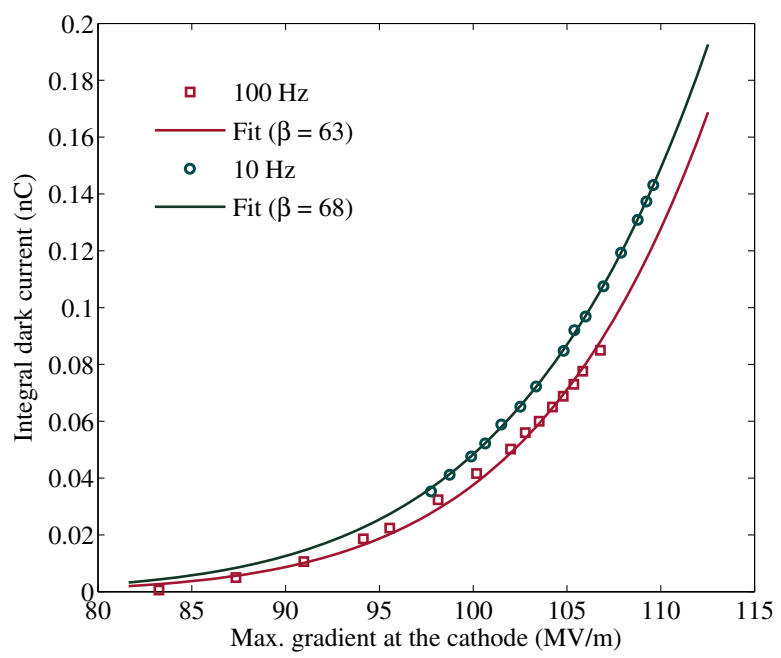

FIG. 19. Integrated dark current per rf pulse as a function of the peak electric field at the cathode at $10 \mathrm{~Hz}$ and $100 \mathrm{~Hz}$ repetition rate (SwissFEL gun).
TABLE V. Main parameters of the S-band booster constantgradient structures. Where available and of relevance measured values are given in brackets.

\begin{tabular}{lr}
\hline \hline Frequency $(\mathrm{MHz})$ & 2997.912 \\
Overall length $(\mathrm{mm})$ & 4150 \\
Phase advance & $2 \pi / 3$ \\
Attenuation $(\mathrm{Np})$ & $0.69(0.68-0.70)$ \\
Filling time $(\mathrm{ns})$ & $955(960-970)$ \\
Gradient/power $((\mathrm{MV} / \mathrm{m}) / \sqrt{\mathrm{MW}})$ & $3.22(3.07-3.10)$ \\
Maximum pulse repetition rate $(\mathrm{Hz})$ & 100 \\
Operating temperature $\left({ }^{\circ} \mathrm{C}\right)$ & 40.0 \\
\hline \hline
\end{tabular}

TABLE VI. Energy gains provided by the S-band rf plants and corresponding klystron operational parameters.

\begin{tabular}{lcccc}
\hline \hline rf plant & $\begin{array}{c}\text { Energy } \\
\text { gain } \\
(\mathrm{MeV})\end{array}$ & $\begin{array}{c}\text { High } \\
\text { voltage } \\
(\mathrm{kV})\end{array}$ & $\begin{array}{c}\text { Anodic } \\
\text { current } \\
(\mathrm{A})\end{array}$ & $\begin{array}{c}\text { Output } \\
\text { power } \\
(\mathrm{MW})\end{array}$ \\
\hline FINSS (gun) & 7.1 & 235 & 225 & 17.0 \\
FINSB01 & 56.3 & 235 & 262 & 20.8 \\
FINSB02 & 75.6 & 285 & 312 & 37.5 \\
FINSB03/04 & $2 \times 60.8$ & 302 & 327 & 48.5 \\
\hline \hline
\end{tabular}

modulator and a Thales TH 2100L (45 MW) $100 \mathrm{~Hz}$ S-band klystron. After some initial difficulties (see Sec. III) the S-band booster has fulfilled its requirements to provide acceleration and stable energy chirp for the magnetic compression with reasonable reliability. Table VI lists the energy gains supplied to the electron beam by all the S-band rf plants (including the SwissFEL gun) at nominal operation conditions when accelerating the beam at the respective on-crest phases. Also listed are the corresponding klystron operational parameters.

To verify the design gradient-to-power ratios given in Table $\mathrm{V}$ we measured the energy gains as a function of the

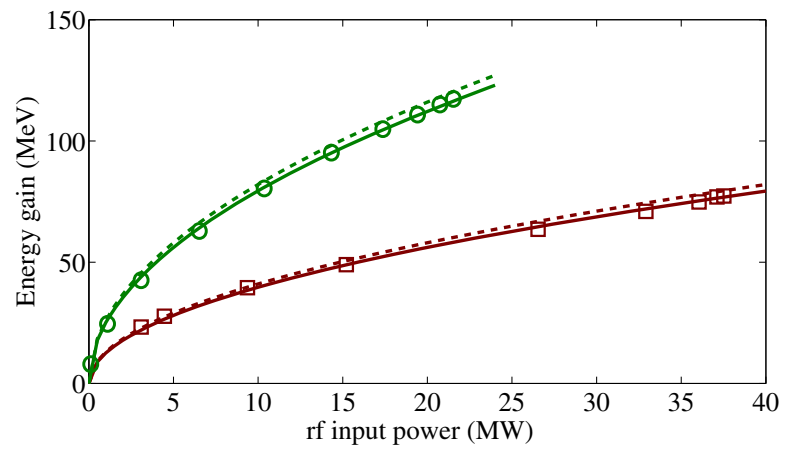

FIG. 20. Measured beam energy gains per accelerating structure as a function of the $\mathrm{rf}$ input power for the FINSB02 (red squares, one structure) and FINSB03/04 (green circles, two structures) rf stations. The solid lines of the corresponding colors represent square-root fits to the data, the dashed lines show the respective design expectations. 
TABLE VII. Main parameters of the X-band constant-gradient structure. Where available and of relevance measured values are given in brackets.

\begin{tabular}{lr}
\hline \hline Frequency $(\mathrm{MHz})$ & 11991.648 \\
Overall length $(\mathrm{mm})$ & 1017 \\
Active length $(\mathrm{mm})$ & 750 \\
Phase advance & $5 \pi / 6$ \\
Attenuation $(\mathrm{Np})$ & $0.50(0.56)$ \\
Filling time $(\mathrm{ns})$ & $100(94)$ \\
Maximum pulse repetition rate $(\mathrm{Hz})$ & 100 \\
Gradient/power $((\mathrm{MV} / \mathrm{m}) / \sqrt{\mathrm{MW}})$ & $7.55(7.15)$ \\
Operating temperature $\left({ }^{\circ} \mathrm{C}\right)$ & 43.5 \\
\hline \hline
\end{tabular}

rf input power for the FINSB02 and FINB03/04 rf plants. The results are shown in Fig. 20. The gradient-to-power ratios of the structures were determined by fitting squareroot functions to the data as $3.07(\mathrm{MV} / \mathrm{m}) / \sqrt{\mathrm{MW}}$ and $3.10(\mathrm{MV} / \mathrm{m}) / \sqrt{\mathrm{MW}}$ for FINSB02 and FINSB03/04, respectively. This corresponds to an integrated accelerating voltage of about $12.5 \mathrm{MV} / \sqrt{\mathrm{MW}}$ for a single structure.

\section{X-band system}

The linearization of the longitudinal phase space needed for a uniform compression of the bunch is provided by a 12-GHz structure (X-band, fourth harmonic of S-band) installed upstream of the bunch compressor. The development of the structure was initiated by PSI and CERN [57], and subsequently carried out by a collaboration including also Sincrotrone Trieste [58,59]. The constant-gradient structure consists of 72 cells with a total active length of $750 \mathrm{~mm}$, a phase advance of $5 \pi / 6$ and an average iris diameter of $9.1 \mathrm{~mm}$. Table VII lists the main rf parameters of the structure. Figure 21 shows the results of the field

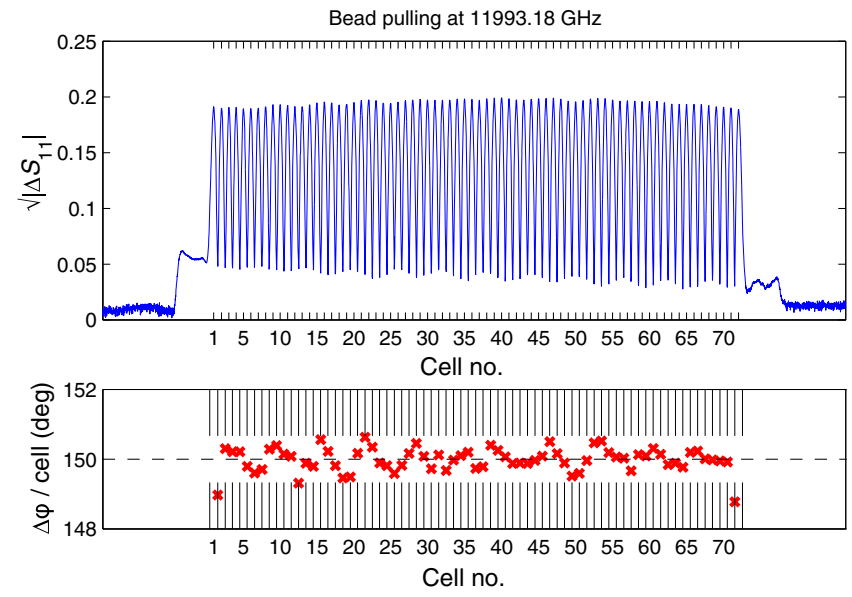

FIG. 21. Field amplitude (top) and phase advance (bottom) measurements of the X-band structure using the bead-pull method, after tuning. Measurements and tuning were carried out at CERN [60]. amplitude and the phase advance measurements using the bead-pull method [60].

The geometry adopted, similar to the one of the NLC type H75 [61], represents a compromise between a high shunt impedance, to be achieved with smaller iris diameters, and a low transverse kick (reduced wakes), calling for larger iris diameters. The input and output coupler design is based on the mode launcher design (see Ref. [62]), which converts a rectangular $\mathrm{TE}_{10}$ waveguide mode into the circular $\mathrm{TM}_{01}$ waveguide mode of the X-band structure. The advantage of this design is a reduction of the surface electric field due to the rounded and thickened matching cell irises and the coupling of the rf power through the broad wall of the feeding waveguide.

The structure integrates two alignment monitors for accurate beam steering and trajectory correction (see Sec. V C). To facilitate beam-based alignment the cavity is placed on a rigid motorized support that can be repositioned remotely by a maximum of $2 \mathrm{~mm}$ with a resolution less than $2 \mu \mathrm{m}$ and a reproducibility better than $\pm 5 \mu \mathrm{m}$. Figure 22 shows a longitudinal cut of the X-band structure with waveguide connection and motorized support.

The associated rf plant is equipped with a ScandiNova K2-3X solid state modulator and a $50 \mathrm{MW}$ and $100 \mathrm{~Hz}$ $\mathrm{X}$-band klystron. The klystron is a SLAC XL5 klystron [63], which is a scaled version of the XL4 tube [64], developed in the framework of a cooperation agreement between SLAC, CERN, PSI and Sincrotrone Trieste.

The ideal operating temperature was found by measuring the beam energy gain as a function of structure temperature. As shown in Fig. 23 (top), maximum acceleration is

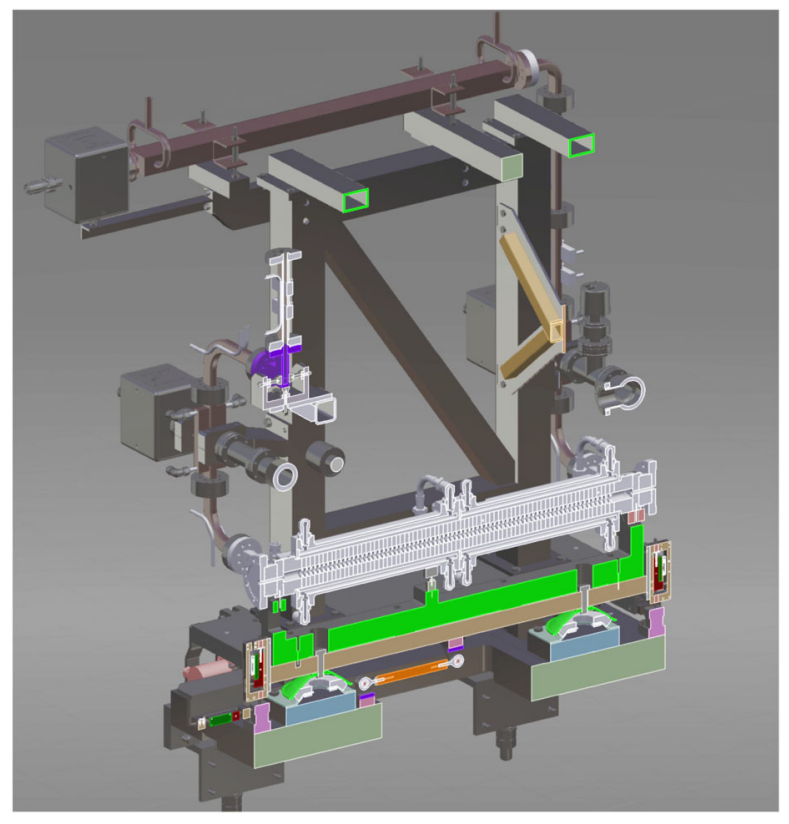

FIG. 22. Cutaway drawing of the X-band structure including waveguide system and motorized support. 

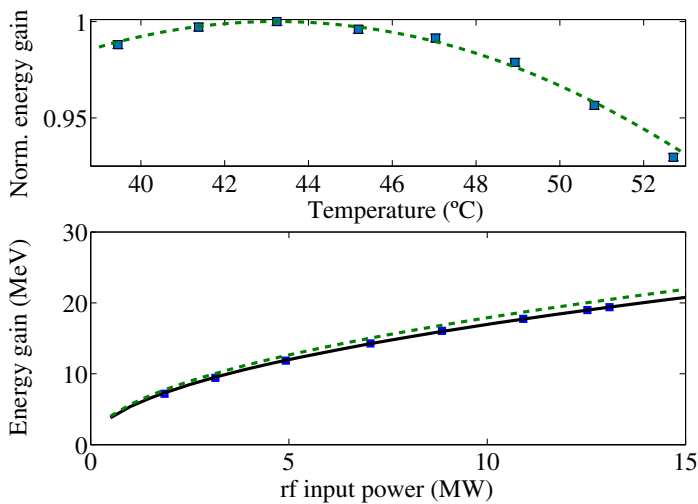

FIG. 23. Measured beam energy gains (blue squares) for the $\mathrm{X}$-band structure as a function of operating temperature (top) and rf input power (bottom). The dashed lines correspond to the design expectation, the solid line is the square-root fit used to extract the structure's gradient-to-power ratio.

obtained at an operating temperature of $43.5^{\circ} \mathrm{C}$. Similar to the S-band structures, we determined the gradient-to-power ratio by recording the beam energy gain as a function of the rf input power (Fig. 23). In the case of the X-band structure, the square-root fit to the measurements yields a ratio of $7.15(\mathrm{MV} / \mathrm{m}) / \sqrt{\mathrm{MW}}$.

\section{S-band transverse deflecting cavity}

In the diagnostics section following the bunch compressor a transverse deflecting rf cavity [65] is used to streak the electron bunches in the vertical direction for diagnostics purposes. The induced correlation between longitudinal and vertical coordinate along the bunch allows measurements of bunch length and current profile with regular transverse profile monitors [66,67].

The deflector is a standing-wave structure consisting of five cells operating in the $\pi$-mode. It was manufactured by INFN Frascati, according to a design that evolved from an earlier one developed for the SPARC project [68] by scaling it to the SITF operating frequency. A summary of the most important parameters is given in Table VIII.

TABLE VIII. Main parameters of the S-band deflecting cavity. Where available and of relevance measured values are given in brackets. The time constant refers to the rise time of the electromagnetic field inside the cavity.

\begin{tabular}{lc}
\hline \hline Frequency $(\mathrm{MHz})$ & 2997.912 \\
Overall length $(\mathrm{mm})$ & 441 \\
Phase advance & $\pi$ \\
Quality factor & $15600(15500)$ \\
Time constant $(\mathrm{ns})$ & $800(855)$ \\
Transverse shunt impedance $(\mathrm{M} \Omega)$ & 2.5 \\
Deflecting voltage/power $(\mathrm{MV} / \sqrt{\mathrm{MW}})$ & $2.24(2.20)$ \\
Maximum pulse repetition rate $(\mathrm{Hz})$ & $100(10)$ \\
Operating temperature $\left({ }^{\circ} \mathrm{C}\right)$ & 40.0 \\
\hline \hline
\end{tabular}

The rf power is provided by a Thales TH 2157 (7.5 MW) $100 \mathrm{~Hz}$ S-band klystron with a ScandiNova K1-2S solid state modulator. The klystron output is connected to an $3.75 \mathrm{~kW}$ average-power (7.5 MW peak-power) rf circulator (AFT microwave). While installed at the SITF, the rf deflector was only operated at $10 \mathrm{~Hz}$ repetition rate. It is, however, planned to use the same cavity at the SwissFEL facility at a rate of $100 \mathrm{~Hz}$. Details on the calibration and the performance of the transverse deflector will be given in Sec. IV F 4.

The initial injector design had provisions for a single-cell transverse deflecting cavity for the longitudinal analysis of the electron bunch emerging from the rf gun [69]. Eventually the plan for such a low-energy rf deflector was not realized at the SITF due to substantial beam optics problems associated with the deflection of relatively long bunches at very low beam energies.

\section{Low-level rf system}

The primary goal of the implementation of a low-level rf (llrf) system at the SITF consisted in testing suitable concepts and operational issues for the SwissFEL facility rather than the validation of the final SwissFEL llrf hardand software.

\section{System overview}

Each of the five S-band and one X-band rf stations at the SITF is controlled by a digital llrf system. The pulsed S-band rf signals from pickups and directional couplers are down-converted to an intermediate frequency (IF) of 46.842 MHz (64th subharmonic of the S-band frequency $2997.912 \mathrm{MHz}$ ). An additional down- and up-conversion stage is used for the X-band system, which translates the $11991.648 \mathrm{MHz}$ signals to S-band frequencies and vice versa. Therefore the same llrf system as for the S-band systems can be used for the X-band system. Local phaselocked oscillator (PLO) units at each rf station generate the required S-band rf frequency and additionally 9 and $12 \mathrm{GHz}$ signals at the X-band rf station. All PLO units are locked to the master oscillator by means of a temperature stabilized coaxial reference distribution system.

The common IF signals are digitized by 16-bit analogto-digital (A/D) converters running at a sampling rate of $124.913 \mathrm{MHz}$ (the 24th subharmonic of the S-band frequency). A so-called non-IQ algorithm [70] implemented in FPGA down-converts the digitized signals to baseband, providing I/Q values of the rf signals. These waveforms cover a time window of about $8.2 \mu \mathrm{s}$. Amplitude and phase waveforms are calculated in firmware and transferred to the EPICS control system input/output controller (see Sec. IV G). The drive signal to the amplifier chain can be controlled by a baseband vector modulator with its I/Q inputs connected to a 16-bit digital-to-analog (D/A) converter with the same output rate as the A/D converters. This allows arbitrary amplitude and phase 


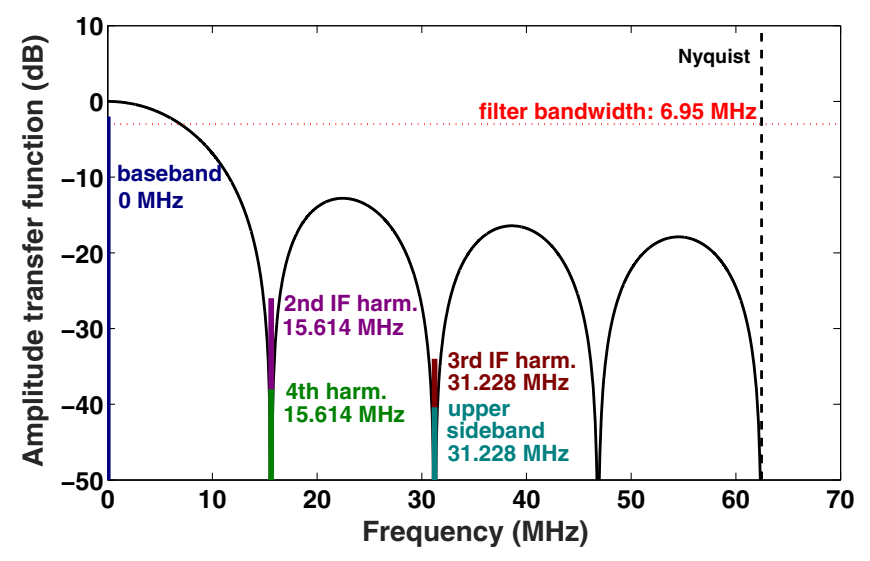

FIG. 24. Amplitude transfer function of the non-IQ digital filter of the llrf system.

waveform shaping of the drive signal within a bandwidth of slightly more than $20 \mathrm{MHz}$.

The implemented non-IQ algorithm with its IF to sampling frequency ratio of $f_{\mathrm{IF}} / f_{s}=3 / 8$ offers the advantage of its simplicity with respect to the digital implementation in firmware, and the benefit that the digitally generated upper sideband in the down-conversion process at $2 f_{\mathrm{IF}}$ as well as harmonics up to 6th order are automatically suppressed by the non-IQ filter characteristics. The amplitude transfer function of the non-IQ algorithm is shown in Fig. 24. The detection bandwidth of the complete llrf system is mainly dominated by this digital filter and covers about $7 \mathrm{MHz}$ ( $-3 \mathrm{~dB}$ bandwidth). It turns out that the choice of IF and sampling frequencies with the implemented down-conversion algorithm is a good compromise between detection bandwidth and achievable resolution under the constraint of the performance of $A / D$ converters at this time.

The proof-of-concept system is realized with FPGA evaluation boards (with Xilinx Virtex-5 FPGA), custom crates and $\mathrm{A} / \mathrm{D}$ as well as $\mathrm{D} / \mathrm{A}$ converter modules. The maximum repetition rate of this setup is limited to $10 \mathrm{~Hz}$ whereas a $100 \mathrm{~Hz}$ processing is required for SwissFEL. Amplitude and phase averages of all acquired rf waveforms are calculated (typically within a window of about $1 \mu \mathrm{s}$ for the S-band structures and $100 \mathrm{~ns}$ for the X-band structure), which are then used in pulse-to-pulse feedback loops implemented at the EPICS level to compensate for slow drifts in the rf system. Due to the very short rf pulse lengths no intrapulse feedback has been realized. The pulse-topulse stability is therefore predominantly depending on the stability of the drive chain. The temperature stability of the traveling-wave structures is maintained to better than $\pm 0.1^{\circ} \mathrm{C}$. Nevertheless, any temperature variation within this range results in a change of rf phase velocity, which then translates into a phase slippage between $\mathrm{rf}$ wave and electron beam. The model for the installed S-band structures is compared to experimental results in Fig. 25.

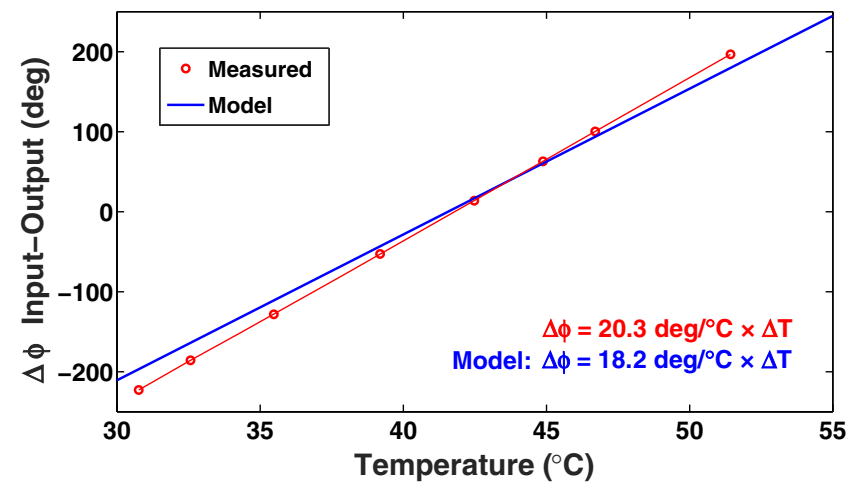

FIG. 25. Phase slippage between output and input of the S-band traveling wave structures due to temperature variations of the structure. The ideal operating temperature, where output and input phases are equal, is found to be $41.7^{\circ} \mathrm{C}$ in this example.

The effective electron beam accelerating phase is very close to the average of in- and output phase of the rf wave. Therefore, all rf based feedbacks are implemented as vector-sum feedbacks to keep constant the phase of the vector sum of those signals.

The llrf system is located in temperature stabilized huts, which provide the same constant temperature as inside the accelerator tunnel to a tolerance of $\pm 0.1^{\circ} \mathrm{C}$. The huts are located directly adjacent to the linac tunnel resulting in maximum cable lengths of $10 \mathrm{~m}$. The phasedrift-compensated 3/8-inch Andrew Heliax rf cables have expected drifts of less than $0.1 \mathrm{ps}$ (about $0.1^{\circ} \mathrm{S}$-band phase) in this entirely temperature stabilized environment.

\section{Operational experience, performance and measurements}

The operation of the llrf system has turned out to be very reliable. Besides the standard control tasks the main duties of the llrf system are to mitigate rf amplitude and phase drifts below the required rf stability tolerances and monitor the pulse-to-pulse jitter of the drive chain, which entirely depends on the stability of the individual components such as rf reference signal, vector modulator, preamplifier and modulator. Table IX gives an overview of the required $\mathrm{rf}$ stability tolerances and the performance of the llrf measurement system for the different frequency bands.

The integrated amplitude and phase of an accelerating structure seen by the beam is closely related to the bandwidth of the structure and thus to the filling time. Therefore, the pulse-to-pulse jitter, reported in Table IX, is calculated within the time window of interest, which is approximately the filling time of the structure. The phase jitter performance of the X-band system is mainly limited by the preamplifier stability. In addition, the X-band klystron cannot be operated in saturation due to missing drive power which then limits the amplitude stability of the forward power. 
TABLE IX. Pulse-to-pulse jitter resolutions of the llrf measurement system for the two frequency bands, compared to SwissFEL tolerances (rms values). The averaging window size for the jitter measurements is $900 \mathrm{~ns}$ (100 ns) for S-band (X-band).

\begin{tabular}{llcc}
\hline \hline Frequency band & \multicolumn{1}{c}{ Parameter } & SwissFEL rf tolerance & Measurement resolution \\
\hline S-band & Amplitude (relative) & $1.8 \times 10^{-4}$ & $4.4 \times 10^{-5}$ \\
& Phase & $0.018^{\circ}$ & $0.0034^{\circ}$ \\
X-band & Amplitude (relative) & $1.8 \times 10^{-4}$ & $1.8 \times 10^{-4}$ \\
& Phase & $0.072^{\circ}$ & $0.019^{\circ}$ \\
\hline \hline
\end{tabular}

Due to the fact that all llrf local oscillator (LO) and clock frequencies are derived from a single rf reference signal at the location of the rf station, the reference signal can be used to determine drifts of the llrf measurement system. It turns out that the main drift originates from the drift of the LO frequency, which can easily be compensated by the socalled "reference tracking" method. The reference signal is split and fed into one of the rf receiver channels. Any observed long-term drift on this channel is subtracted from all other measurement channels thus minimizing the effect of common drifts. Measurements showed that the remaining channel-to-channel drift amounts to less than $0.1^{\circ}$ per day.

The typically achieved stability figures for the injector accelerating structures over one hour of operation are given in Table X. In most cases the obtained stabilities fall short of the SwissFEL requirements for the pulse-to-pulse stability of the drive chain. Upgrades of preamplifiers and high-voltage modulators will be required when reusing them at the SwissFEL facility.

The possibility to drive the output of the vector modulator with arbitrary waveforms offers the flexibility to shape the rf pulse as desired. We use this, for instance, to alleviate the problem of exciting undesired passband modes of the rf gun. During the initial operation of the first rf gun (CTF gun, see Sec. IV C 1) a rectangular shaped pulse of the forward rf power excited the adjacent passband mode, only 8.1 $\mathrm{MHz}$ away from the fundamental $\pi$-mode. Introducing a Hamming shaped window at the raising and falling edges of the forward amplitude avoids this undesired excitation (see Fig. 26).

TABLE X. Overview of typical rf stability figures (rms values) over one hour of operation for all rf stations operated at the SITF.

\begin{tabular}{lll}
\hline \hline rf station & Amplitude & Phase \\
\hline S-band: & & \\
FINSS (rf gun) & $1.3 \times 10^{-4}$ & $0.020^{\circ}$ \\
FINSB01 & $1.4 \times 10^{-4}$ & $0.030^{\circ}$ \\
FINSB02 & $1.2 \times 10^{-4}$ & $0.027^{\circ}$ \\
FINSB03/04 & $2.1 \times 10^{-4}$ & $0.032^{\circ}$ \\
$X$-band: & & \\
FINXB $^{\text {a }}$ & $2.1 \times 10^{-3}$ & $0.17^{\circ}$ \\
\hline \hline
\end{tabular}

${ }^{\mathrm{a}}$ Limited by klystron preamplifier stability.

\section{Outlook for the SwissFEL low-level rf system}

Based on the experience gained with the operation of the prototype llrf system at the SITF several improvements are planned for the SwissFEL series systems. The digital processing platform will be changed to a decentralized system. Several standard VME64x PowerPC boards with commercial off-the-shell A/D and D/A FPGA mezzanine cards (FMC standard) will provide a well-defined future hardware upgrade path and will remove bottlenecks in the data acquisition chain to comply with operation at the $100 \mathrm{~Hz}$ pulse repetition frequency. Furthermore, the operation of a large number of almost identical rf stations calls for automated approaches for startup procedures, operating point determinations, failure analyses and recovery actions [71]. A first prototype of the new SwissFEL 1lrf system has been installed and tested at the SwissFEL C-band rf test stand at PSI [72].

\section{E. Bunch compression chicane}

Bunch compression is achieved with a four-bend magnetic chicane, where the two central dipole magnets are installed on a motorized movable girder to allow for different deflection angles. The bunch compressor support unit consists of a system of girders made of granite, a material with excellent thermal stability. The bending magnets and the beam-line elements are mechanically linked by movably mounted steel girders. This mounting concept, called "train link," provides an exact mechanical reference for the beam transport through the dipole magnets as well as for the adjustment of diagnostics components. The construction design of the bunch compressor unit was optimized for stability against static and dynamic stress, taking into account the structure's natural frequencies and possible external excitations such as earthquakes.

The four dipole magnets are powered in series to ensure an equal field in each dipole. Small, individually powered corrector dipoles are integrated in the main dipoles with the original purpose of compensating possible field imperfections. Since these imperfections turned out to be small, the corrector dipoles are mainly used to compensate the geomagnetic field, see Sec. VI A 4. Two small quadrupoles attached to the outer arms are used to correct for residual dispersion. In March 2013, additional skew quads were added to enable studies related to beam-tilt correction based 

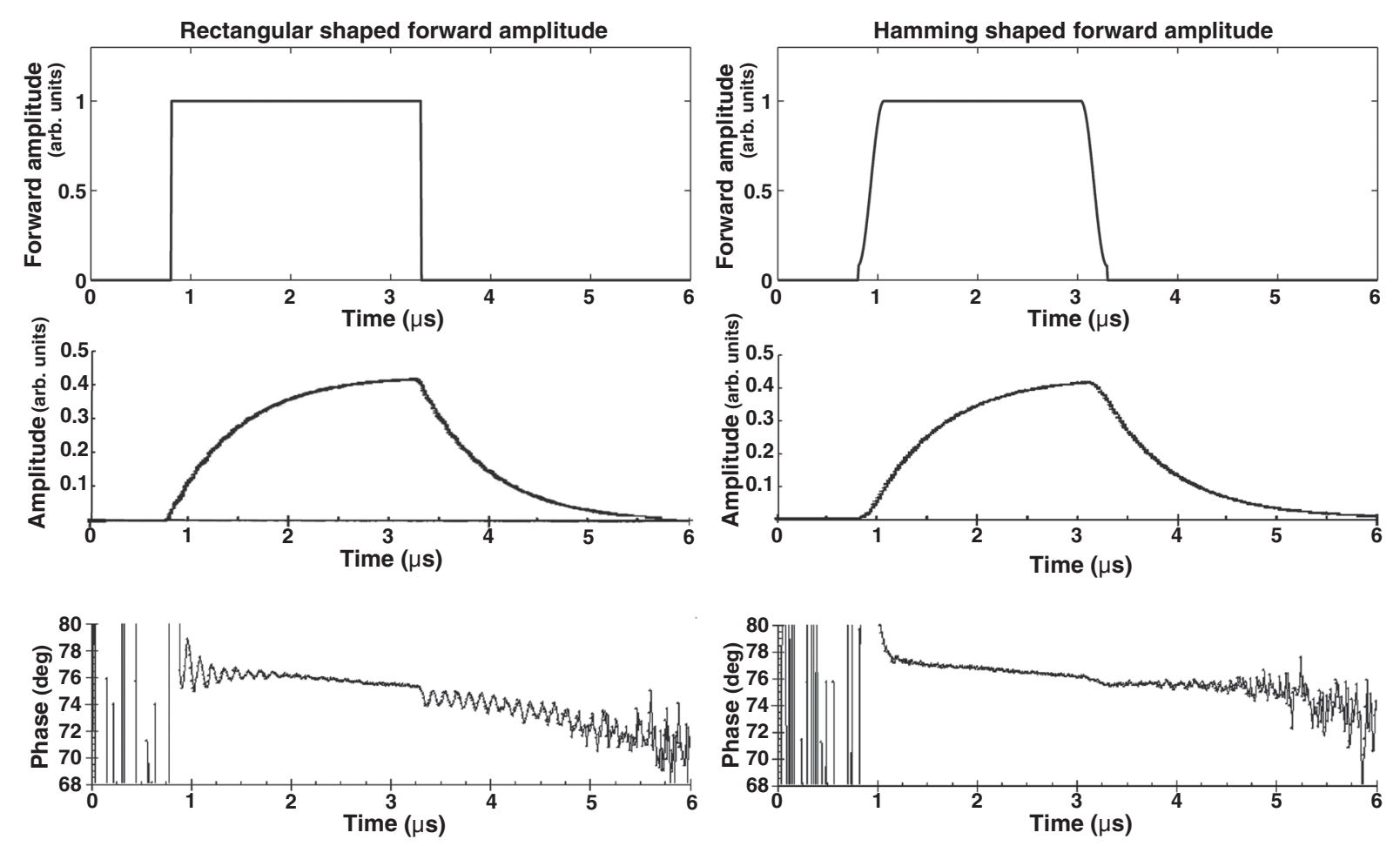

FIG. 26. Gun rf amplitude and phase with "rectangular" (left plots) and with "Hamming" (right plots) rf pulse shape excitation. The rectangular shaped forward pulse also excites the nearest undesired mode $8.1 \mathrm{MHz}$ away from the $\pi$-mode.

on dispersion [73]. The transverse profile of the dispersed beam can be observed by means of a movable YAG:Ce screen in the central chamber of the chicane, which is imaged by a CCD camera with a low-resolution lens. More detailed information on the beam can be obtained, in a nondestructive manner, from the synchrotron radiation monitor described in Sec. V D 2. Two horizontally movable beam scrapers, also installed in the central chamber, can be used to collimate the dispersed beam and thus select a certain energy range. Figure 27 shows a 3D drawing of the

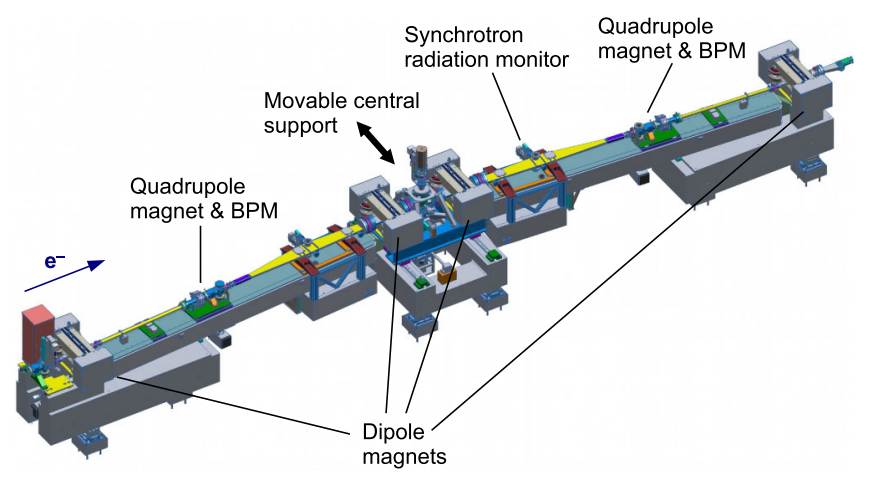

FIG. 27. 3D drawing of the bunch compression chicane showing the initial configuration featuring dipole magnets with return-yoke windings. The thick black arrow indicates the lateral movement of the central support for the two inner dipole magnets, which also houses a screen monitor and insertable collimation masks. compression chicane, Table XI lists the most relevant parameters.

The large dispersion in the central region of the bunch compressor gives rise to a transverse beam size of several millimeters and orbit excursions within the dipole fields of similar magnitude. Therefore a wide transverse good-field region in the main dipole magnets of the chicane is essential. The original design of the bunch compressor dipole magnets, shown in Fig. 28 (top), is a traditional H-type magnet with coil windings around the upper and lower poles, providing a good-field region of $60 \mathrm{~mm}$ after shimming. (Here, the good-field region is defined according to the requirement $\int(\Delta B / B) d s<10^{-4}$.) Following heightened concerns about a possible emittance growth due to nonlinear dispersion and nonlinear focusing effects in the dipole fields based on experience gained at LCLS [74], a second design with a larger pole width was proposed and

TABLE XI. Main bunch compressor parameters.

\begin{tabular}{lcc}
\hline \hline Parameter & Nominal & Maximal \\
\hline$R_{56}$ & $-46.8 \mathrm{~mm}$ & $-68.9 \mathrm{~mm}$ \\
Displacement & $0.333 \mathrm{~m}$ & $0.404 \mathrm{~m}$ \\
Deflection angle & $4.07^{\circ}$ & $5.0^{\circ}$ \\
Bend magnetic field & $0.18 \mathrm{~T}$ & $0.22 \mathrm{~T}$ \\
Total length & & $11.16 \mathrm{~m}$ \\
Inner drift length & & $0.75 \mathrm{~m}$ \\
Outer drift length & $4.39 \mathrm{~m}$ \\
Dipole length (effective) & & $0.25(0.303) \mathrm{m}$ \\
\hline \hline
\end{tabular}



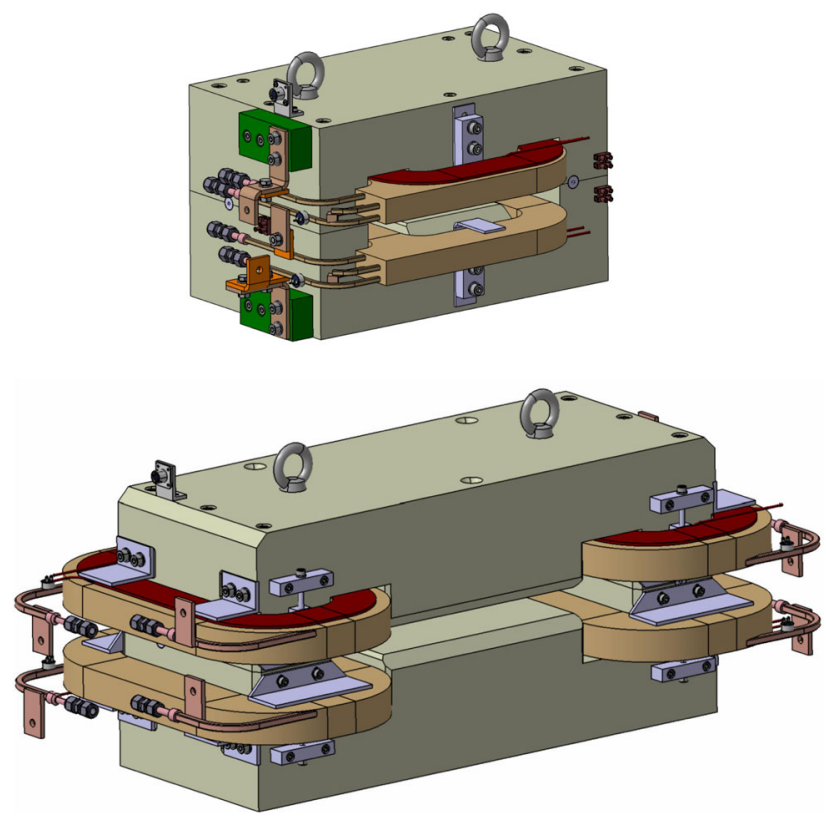

FIG. 28. The two dipole magnet designs tested in the SITF bunch compressor. Top: original design with pole windings. Bottom: second design with larger pole width, later discarded because of the long-range stray fields resulting from the returnyoke windings.

realized (Fig. 28, bottom). By arranging the coils in two pairs, each around the return yokes of the H-type magnet, it was possible to extend the size of the transverse good-field region to $110 \mathrm{~mm}$ [35].

The initial bunch compression studies at the SITF were carried out with dipole magnets of this second type, providing the larger good-field region. Unfortunately, however, the beam measurements revealed that the magnets with the return-yoke windings exhibit a long-range stray field, perturbing the beam orbit in locations as far as as $2 \mathrm{~m}$ before the bunch compressor entrance. While bunch compression studies at the SITF were still possible with suitable orbit corrections, the impairment by the stray fields was deemed unacceptable for the future operation of SwissFEL. As an obvious alternative to examine, the originally foreseen dipole magnets with coil windings around the poles were installed in a brief shutdown in July 2014 (see Sec. III D). Subsequent measurements performed with these dipole magnets exposed no problems related to the magnet design, either from stray fields or due to the limited good-field region.

Based on the experience gained at the SITF, the SwissFEL bunch compressors will be equipped with dipole magnets of the first design, i.e., with coil windings around the poles. Furthermore, as a consequence of the observed susceptibility of the transverse beam emittance to coherent synchrotron radiation effects (see Sec. VIIC for details), the compression chicanes at SwissFEL will be somewhat longer than that at the SITF $(13.75 \mathrm{~m}$ and $16.95 \mathrm{~m}$ for the first and second chicane, respectively, as compared to $11.16 \mathrm{~m}$ at the SITF).

\section{F. Electron beam diagnostics}

Here we briefly review the main diagnostics systems used for the routine operation and beam characterization tasks at the SITF [75], namely charge monitors (Sec. IV F 1), beam position monitors based on resonant striplines (Sec. IV F 2), screen monitors (Sec. IV F 3) and the combination of transverse deflecting rf cavity and screen monitor for longitudinal diagnostics (Sec. IV F 4). Additional diagnostics systems not used for routine operation but nevertheless profiting from the available electron beam for test purposes will be described in Sec. V.

\section{Charge monitors}

The determination of the bunch charge along the beam line is essential to ensure full transmission and for the correct interpretation of all beam physics measurements. At the SITF we mainly rely on the strip-line BPMs, to be described in Sec. IV F 2, for the monitoring of the bunch charge. While the BPMs provide a rather precise relative measurement of the bunch charge, they cannot give an absolute charge reading and need to be calibrated with an independent charge measurement. For the absolute charge measurement, a wall current monitor in the gun section is used. This monitor measures the voltage across $3 \Omega$ resistors around the circumference of a ceramic gap in the beam pipe, which is proportional to the wall current flowing across the gap. At our typical bunch charges this voltage is very small, leading to a readout noise equivalent to about $10 \mathrm{pC}$. With its bandwidth of several $\mathrm{GHz}$ the wall current monitor is capable of distinguishing the photoelectron beam from the dark current.

In the same section, an insertable coaxial Faraday cup is also available for charge measurements. It is primarily used for dark current measurements (see Sec. IV C 1). Both the wall current monitor and the Faraday cup are based on designs already applied at the Swiss Light Source (SLS) [76]. No dedicated read-out system has been devised for these two monitors-they are simply read out with oscilloscopes.

At SwissFEL cavity BPMs (see Sec. V B) will be used primarily to monitor variations in bunch charge, along with integrating current transformers (ICT) for absolute charge measurements. A few ICTs, both of the standard kind and prototypes, have been installed and tested at the SITF to assess their performance in view of the SwissFEL requirements. These tests are described in Sec. VA.

\section{Resonant stripline beam position monitors}

The significantly different requirements and time scales of the SITF and SwissFEL projects led to the development of two separate beam position monitor (BPM) systems for 

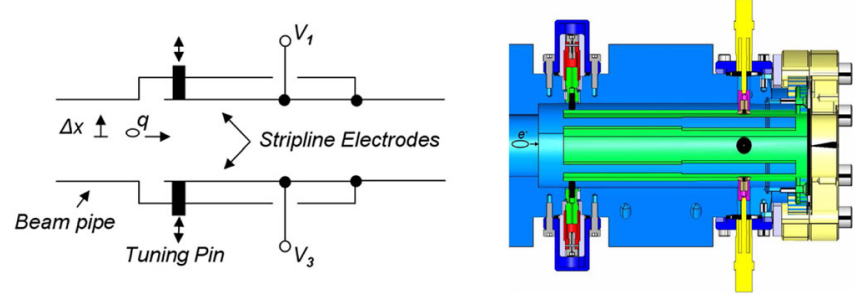

FIG. 29. Resonant stripline BPM pickup schematics (left) and mechanical drawing (right) [80].

the two machines. To cover the need for a robust and reliable BPM system operational from day one, a system of 19 resonant-stripline BPMs based on proven technology and existing implementations at PSI was built for the SITF [77]. The use of button BPMs was discarded due to their inferior resolution at low bunch charges [78]. At the same time, the development of an advanced cavity BPM system was started, aiming to fulfill the much more stringent requirements of the SwissFEL facility arising from the varying beam pipe diameter, the presence of an undulator section and other factors [79]. For this development, a dedicated test area was made available at the SITF, located in the downstream part of the facility. Here we describe the resonant-stripline BPM system. The tests of the cavity BPM system performed at the SITF are summarized in Sec. V B.

The pickups (see Fig. 29) contain four strips mounted parallel to the beam direction, each with an open and a shorted end $(\lambda / 4$ resonator configuration). The inner diameter of the pickup corresponds to the beam pipe dimension of $38 \mathrm{~mm}$. A passing electron bunch excites a decaying sine signal at $500 \mathrm{MHz}$ on each of the strips (Fig. 30). The design of the pickups is based on earlier monitors in use at the linac and transfer lines of the SLS at PSI, but was optimized for the use at the SITF, for instance by adding mechanical resonance frequency tuners [80]. Moreover, profile monitors (not shown in Fig. 29) were integrated into the BPM enclosures to save costs and to

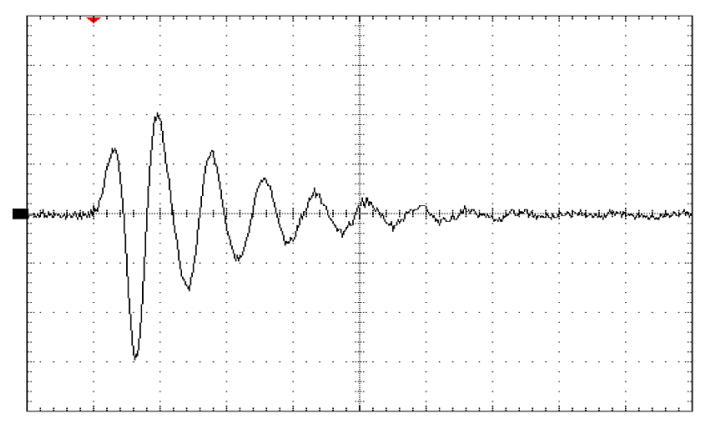

FIG. 30. Beam-induced resonant stripline BPM pickup signal voltage (vertical axis, $1 \mathrm{~V} /$ div) versus time (horizontal axis, $2.5 \mathrm{~ns} / \mathrm{div}$ ), sampled with a 20 GSample/s oscilloscope (screenshot). Only the $500 \mathrm{MHz}$ fundamental mode is visible, higher harmonics are suppressed with a low-pass filter. enable straightforward cross calibration between view screen and BPMs. The choice of $500 \mathrm{MHz}$ as fundamental resonance frequency of the resonant stripline pickup created synergies with present and future BPM systems at the SLS, which operate at the same frequency [81].

The four stripline signals are coupled out via antennas near the shorted ends of the strips before being filtered and amplified in an rf front-end (rffe) board. The four rffe output signals are connected to a digitizer board, consisting of a mezzanine module for analog-to-digital conversion, which is plugged onto a FPGA carrier board for digital signal processing and calculation of beam position and charge (Fig. 31). The FPGA carrier board design was already used for other systems at PSI, e.g., for proton accelerator BPM systems [82], or for the MEG particle physics experiment at PSI [83]. In the latter application, the mezzanine modules of the FPGA board feature an analog sampling chip called DRS4 (domino ring sampler version 4) [84], which was developed at PSI for the cost-effective digitization of several thousand detector signals at rates of up to $5 \mathrm{GSample/s}$. Since the chip is well suited for sampling $500 \mathrm{MHz}$ resonant stripline pickup signals, we use it for our BPM readout electronics, accommodated in newly developed mezzanine modules optimized for the BPM system. The analog input stage of this mezzanine module has bandpass filters and variable gain amplifiers for each of the four input channels, leading to an overall BPM system gain range of $63 \mathrm{~dB}$.

The modest cost of the DRS4 chip allows it to be used in a redundant way. For instance we only use four of the nine available input channels on each chip (one per stripline). Using only every second channel reduces crosstalk, which is most pronounced between neighboring channels. Moreover the four stripline signals are read out independently by two chips. This not only improves the noise at higher bunch charge by averaging the readings of both chips, but also improves the mean time between failures of the system, enabling the detection of rare performance

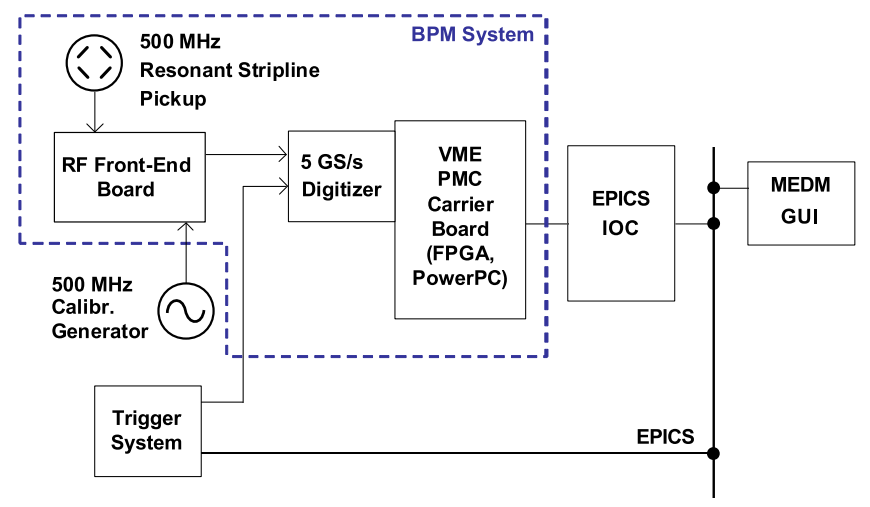

FIG. 31. BPM system block schematics, including the linkage to the external trigger system and the EPICS control system (see Sec. IV G) [77]. 
degradations or failures of the DRS4 chips (2.5\% failure rate in 4 years of SITF operation).

Each of the two DRS4 chips on the mezzanine records the four BPM signals with 5 GSample/s and stores the signal waveforms internally in an array of 1024 sampleand-hold $(\mathrm{S} / \mathrm{H})$ capacitors per channel. The sampling (i.e., the domino wave) is stopped for the readout of the waveforms stored in the S/H cells. For each channel, a dedicated multiplexer inside the chip reads out the voltage of each $\mathrm{S} / \mathrm{H}$ cell one by one. The signal is then digitized using a commercially available multichannel ADC working at $33 \mathrm{MSample} / \mathrm{s}$, connected to the multiplexer output of the DRS4 chip.

The resonant-stripline BPMs require an external trigger from the timing system to ensure the measurement of the BPM signal at the right time. During the initial commissioning phase, which involved frequent changes in the gun laser or synchronization setup, the actual bunch arrival time could vary with respect to the external trigger by several nanoseconds on short timescales. Since the BPMs automatically detect the peak of the envelope waveforms, they can reliably measure beam position and charge even in the presence of arrival time variations exceeding $100 \mathrm{~ns}$. In the event of even larger shifts of the BPM trigger relative to the arrival time (e.g., due to major modifications of the gun laser or the timing system), the beam signal ends up outside of the $\approx 200$ ns sampling window of the BPMs. In this case, a manual readjustment of the BPM or gun trigger delay based on an independent signal, e.g., from the wall current monitor, or from the oscilloscope readout of a BPM pickup, is needed.

The design principle of the DRS4 chip causes the input signals to be sampled at nonequidistant points in time. This so-called time nonlinearity is systematic and mostly reproducible, therefore it can be calibrated in the lab. To this end we use a novel method in which 500 waveforms of a $500 \mathrm{MHz}$ continuous-wave (CW) sine signal are acquired at different phases, where a fit procedure determines the time differences between the waveform sampling points [77]. Further lab calibration is needed for the individual voltage gains and offsets for each $\mathrm{S} / \mathrm{H}$ cell of the DRS4 chip. During beam operation the FPGA then uses the calibration data to apply the appropriate time, gain and offset corrections to the measured BPM signals in real time.

The digital signal processing algorithm on the FPGA board is implemented in a mixture of VHDL (performed in the FPGA fabric) and C code (executed on a PowerPC 405 processor embedded in the FPGA). The FPGA on the carrier board calculates the beam position and charge from the sampled waveforms [shown in Fig. 32(a)] in several steps. First, the gain and offset of each sample are corrected using the calibration look-up table. Then the waveform, consisting of nonequidistant samples, is converted to a waveform with equidistant samples by use of a cubic spline interpolation algorithm.
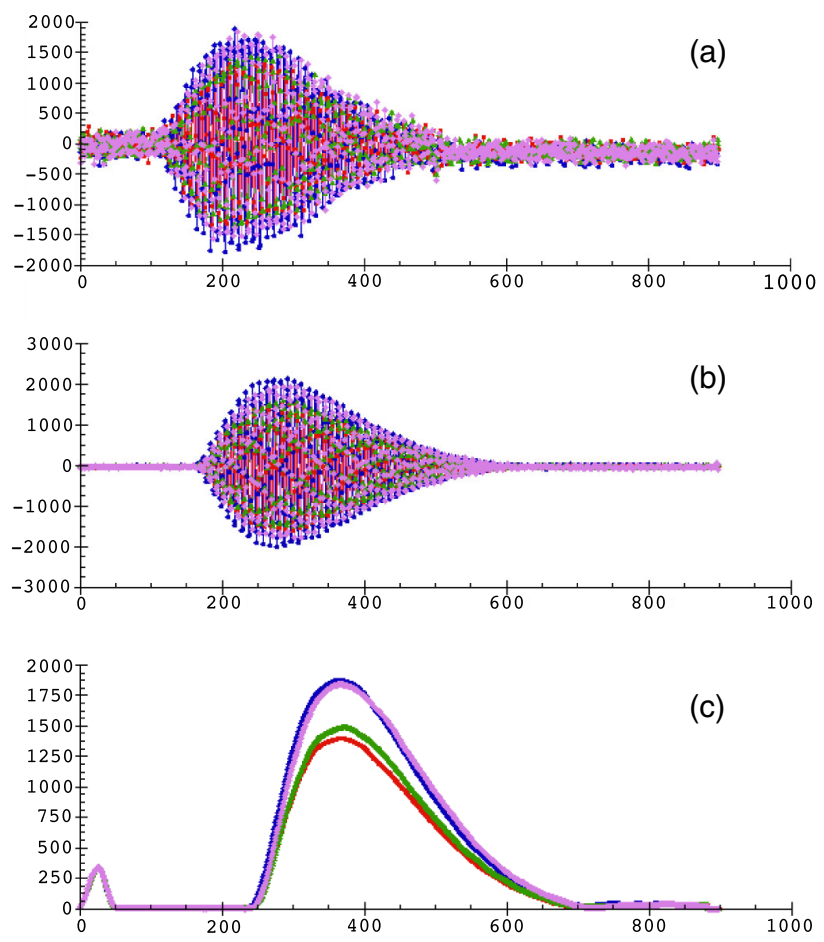

FIG. 32. Raw BPM electronics waveforms (ADC raw value versus sample number), sampled by the DRS4 chip at 5 GSample/ $\mathrm{s}$ (a). The same waveforms after offset gain and time-nonlinearity correction, performed by the FPGA for each sample individually (b). Envelopes of the four BPM electronics signals as calculated by the FPGA (signal amplitude versus sample number) (c). The additional delay is due to digital filtering.

The resulting waveform [shown in Fig. 32(b)] is then bandpass-filtered with a $500 \mathrm{MHz}$ finite impulse response (FIR) filter to suppress noise and higher harmonics. For the filtered waveform, the FPGA reconstructs a $90^{\circ}$ phase shifted signal (Q) from the original signal (I) via Hilbert transform, performs a Cartesian-to-polar conversion of the resulting I/Q vectors, and applies a low-pass filter to obtain the envelopes of the rffe output signal waveforms [shown in Fig. 32(c)]. For the first 50 samples of these waveforms, the pipeline of the FIR filter in the FPGA is not yet fully populated with valid samples, resulting in the artificial signal peak around sample No. 25. For this reason only samples with index greater than 50 are used for further processing.

The signal amplitude of each envelope is then determined from the envelope waveform by integrating over an adjustable number of samples. A number of about 200 samples was empirically found to result in the best resolution. The position of the integration window is dynamically adjusted by the FPGA during operation: The FPGA detects the peak of the envelope and starts the integration a certain number of samples before.

Finally, the horizontal and vertical beam positions, $x$ and $y$, are calculated from the signal amplitudes (i.e., the 
integrals) A, B, C, and D of the four channels according to the approximate formulas

$$
\begin{aligned}
& x=k \frac{(B+C)-(A+D)}{A+B+C+D}, \\
& y=k \frac{(A+B)-(C+D)}{A+B+C+D},
\end{aligned}
$$

with the constant $k=16.2 \mathrm{~mm}$.

Similarly, the bunch charge $Q$ is obtained as

$$
Q=c_{\text {abs }} c_{\text {gain }}(A+B+C+D),
$$

where $c_{\text {gain }}$ is a scaling factor that depends on the (variable) gain of the electronics, and $c_{\mathrm{abs}}$ is a constant scaling factor obtained individually for each BPM by cross-calibration with a dedicated, absolutely calibrated charge monitor-at the SITF normally the wall current monitor (see Sec. IV F 1).

Position and charge resolutions at bunch charges above about $5 \mathrm{pC}$ are dominated by random sampling jitter of the DRS4 chip and thus independent of the bunch charge [77]. To reduce the impact of this jitter on the position resolution of the BPMs, the relatively short pickup signals are stretched in the rffe by applying a narrow-band $500 \mathrm{MHz}$ filter realized in stripline technology on addon printed circuit boards (PCBs). By choosing a filter quality factor about four times higher than the loaded quality factor of the pickups, the number of usable ADC samples for the position calculation is also increased by a factor of four, thereby reducing position and charge noise by a factor of about two. A further reduction in sampling noise is achieved by the parallel sampling of all signals with two DRS4 chips mentioned above.

The position and charge resolutions are determined by correlating the data of several BPMs. For charges above $5 \mathrm{pC}$, we measured a charge-independent position resolution of typically $7 \mu \mathrm{m}$ (rms). For very low bunch charge, the resolution is dominated by thermal rffe noise and thus scales inversely with the charge, with a product of charge and resolution of about $30 \mu \mathrm{mpC}$. This is nearly seven times better than current state-of-the-art button BPM systems operating at similar beam pipe diameters [78]. The relative charge resolution is generally $0.13 \%$ at higher bunch charges (e.g., $130 \mathrm{fC}$ resolution at $100 \mathrm{pC}$ charge). At very low bunch charges the resolution is again dominated by thermal rffe noise leading to an absolute charge resolution between 3 and $4 \mathrm{fC}$. Since the charge resolution of the BPMs is far superior to that of any dedicated charge monitor available at the SITF, beam operation mainly relied on the BPM system for charge measurements, using the dedicated charge monitors only for gun studies or crosscalibration of the BPM charge readings.

\section{Screen monitors}

Among the three types of transverse profile monitors installed in the SITF, wire scanners, synchrotron radiation monitors, and screen monitors, only the last were routinely used for beam optics measurements. The commissioning of the wire scanner and synchrotron radiation monitor systems performed at the SITF primarily served as development work for SwissFEL and will be described in Sec. V D.

View screens provide direct two-dimensional information on the beam profile and play an important role, both in the commissioning and the fine tuning of an electron linac: they are simple and efficient beam finders at the start-up of the machine and later precise and fast tools to characterize the phase space of the beam via optics dependent measurements, including the longitudinal phase space in case a transverse deflecting structure and a dispersive section are available.

The screen monitors of the SITF are combined scintillator and OTR screen monitors including wires for transverse profile scanning in a single device [85]. One OTR screen, consisting of an aluminum-coated silicon mirror, one scintillator, one alignment target, and three $25 \mu \mathrm{m}$ tungsten wires (one horizontal and two diagonal) are mounted on a linear ultra-high-vacuum feedthrough and can be inserted into the beam by means of a stepper motor (see Fig. 33). The scintillator consists either of cerium doped yttrium aluminum garnet (YAG:Ce) or cerium doped lutetium aluminum garnet (LuAG:Ce), the latter offering a twice as large light output at the expense of higher beam losses. Crystal thickness varies between

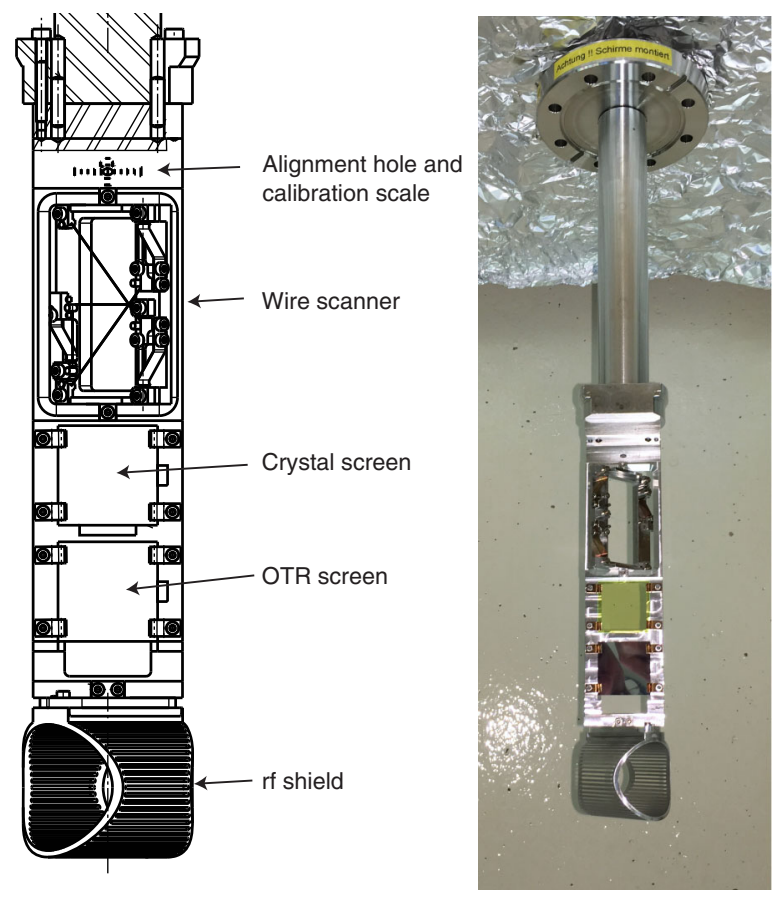

FIG. 33. Technical drawing and photograph of the ultra-highvacuum feedthrough insertion accommodating alignment targets, three scanning wires, two screens (crystal scintillator and OTR), and an rf shield. 
$20 \mu \mathrm{m}$ and $1 \mathrm{~mm}$ depending on the specific detection efficiency and optical resolution needs at a given location.

In the low-energy sections of the machine, only scintillator screens are installed since OTR screens would not yield sufficient signal. These screens are installed perpendicular to the electron beam and viewed through mirrors at $45^{\circ}$ incidence angle, placed directly behind the scintillators. At higher energies scintillator and OTR screens are mounted at an angle of $45^{\circ}$ with respect to the electron beam. Backward transition radiation is thus emitted perpendicular to the electron beam direction. For convenience, the scintillating crystal can be moved to the same location as the OTR screen, such that a realignment and refocusing of the camera is not required. Two cameras with different magnifications are installed, one for overview images $(M=-1 / 5.3)$, and one with a larger magnification $(M=-1 / 1.1)$ of the center of the screen.

Image acquisition was initially performed by FireWire (IEEE 1394 bus standard) cameras, with most of the FireWire network installed in the accelerator tunnel. This setup unfortunately resulted in frequent system crashes requiring manual reboots of the cameras. Moreover, the FireWire solution would not work at the $100 \mathrm{~Hz}$ repetition rate envisaged for SwissFEL. Therefore, new camera types based on the GigE Vision and Camera Link standards have been tested at the SITF and are now planned for use at SwissFEL.

A total of 26 of such monitor stations were installed in the SITF in the beginning and used extensively for the initial setup of the beam optics as well as for early emittance optimizations. Despite the solid performance of these profile monitors, certain limitations suggested a redesign for SwissFEL. First, OTR screens are not suited for the observation of microbunched beams due to the occurrence of coherent optical transition radiation (COTR), as evidenced by measurements performed at LCLS [86], FLASH [87], and, more recently, SACLA [88] (see also Ref. [89]). Since the orientation of the scintillating crystals matches that of the OTR screens, the scintillators would not be exempt from COTR either. Second, the horizontal resolution of the scintillators is limited by the crystal thickness, as even an arbitrarily thin beam would mark a line in the volume of the scintillating crystal. Given that electron beams at SwissFEL are expected to be a few tens of micrometers in diameter, and that scintillator crystals below $100 \mu \mathrm{m}$ thickness are difficult to produce and handle (in addition to providing only weak signal at low charge), this limitation would dominate the resolution in the central regions of the screens. To overcome these shortcomings, the design of the transverse profile monitors was revised. We describe the new design and its use at the SITF in Sec. V D 3.

\section{Longitudinal diagnostics}

The primary technique applied at the SITF for longitudinal beam diagnostics, i.e., bunch length measurements, current profiles, time-resolved ("slice") emittance evaluations, involves the use of the transverse deflecting rf cavity and a transverse profile monitor, typically a view screen, located at an appropriate distance. Technical details on the rf deflector can be found in Sec. IV C 4, while the screens are described in Secs. IV F 3 and VD 3. More advanced, and in particular nondestructive longitudinal diagnostics schemes based on coherent bunch radiation or electro-optic methods were the subject of vigorous research and development efforts at the SITF and will be described in Sec. V.

During normal beam operation, the deflecting rf cavity is powered on, but the timing of its rf pulses is shifted in such a way that the electron bunches traverse the cavity completely unaffected. Only when a longitudinal measurement is required, the timing of the deflector pulse is adjusted to coincide with the passage of the electron bunches. The rf phase is tuned to synchronize the bunch centroid with the zero crossing of the transverse deflecting rf field. Thus, depending on the applied rf power, the bunch is more or less streaked in the vertical plane without deflection of its centroid. The resulting correlation between the electrons' time coordinates and their vertical positions gives access to longitudinal measurements via normal screens $[66,67]$.

A calibration of the setup is easily achieved by relating a change in rf phase (in this case S-band frequency) to the corresponding displacement of the beam centroid on the observing screen. In Fig. 34 we show a comparison between measured and analytically expected calibration factor (conversion of $\mathrm{mm}$ on screen to ps along the bunch) and time resolution as a function of the integrated deflecting voltage. The analytical model agrees well with experimental data. At the highest deflection voltage of $5 \mathrm{MV}$ the time resolution is approximately $12 \mathrm{fs}$ [90].
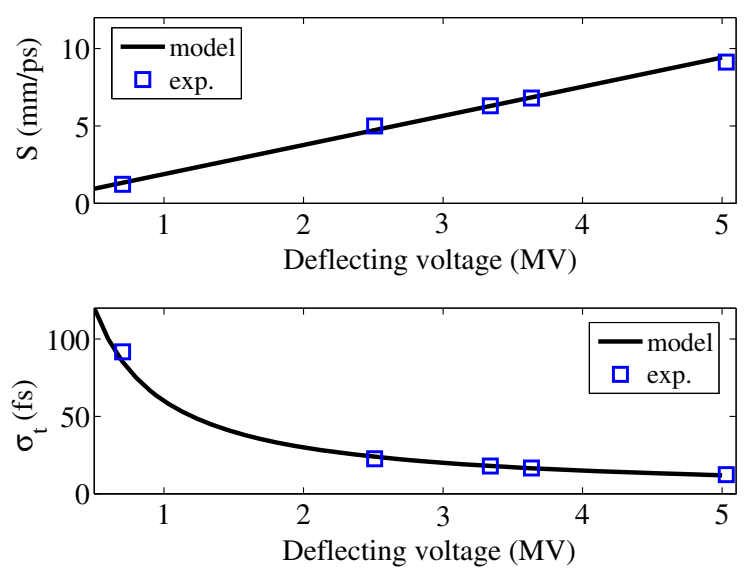

FIG. 34. Deflector calibration factor (top) and time resolution (bottom) as a function of the integrated deflecting voltage, measured in each case at the zero crossing of the rf phase, for a beam energy of $200 \mathrm{MeV}$ and compared to model expectation [90]. 
Other methods to streak the beam for time-resolved measurements exist and have been investigated at the SITF. Instead of an actively applied rf field, the beam induced wakefields in an appropriately shaped dielectric structure may be utilized to generate the desired correlation between the longitudinal and a transverse coordinate. For a report on corresponding beam experiments performed at the SITF we refer to Ref. [91]. The same type of correlation can be achieved by means of dispersion combined with energy chirping. We will briefly expand on this technique during the discussion of time-resolved measurements, see Sec. VI C.

\section{G. Control system}

The SITF control system [92,93] is implemented in a way that allows incremental expansions and upgrades as the project proceeds. With this approach new requirements could be accommodated and new hardware could be incorporated in the course of the different project phases. In general the system is based on well-proven technologies and concepts used at existing PSI facilities, in particular the Swiss Light Source (SLS) [94]. Following this working example it is built around the Experimental Physics and Industrial Control System (EPICS) tool kit [95]. The hardware and software platforms, boot and archive services, configuration management and logistics already developed for the other PSI accelerators are thereby used as far as possible. The linear geometry of the SITF, however, required several adaptations of the existing solutions and led to new designs and concepts for SwissFEL. In the following we describe our approach, with particular emphasis on new developments for SwissFEL.

\section{EPICS}

The use of EPICS not only provides benefits from in-house know-how and the experience gained with other PSI facilities, but also, through its collaborative nature, allows us to tap into the broad pool of expertise accumulated at other laboratories. The SITF control system is based on EPICS version 3.14.8.

The control-room consoles run EPICS client applications, which connect over an Ethernet network to distributed EPICS servers called input/output controllers (IOCs). The IOCs have a direct connection to the hardware devices to be controlled. In addition, central services like the archiver run on dedicated servers located in a server room. On all these computers customized programs required for controlling and characterizing the machine can be implemented and integrated into EPICS.

The most relevant parts of the EPICS toolkit used at the SITF are: (i) the network-based client-server model implementing Channel Access as network protocol; (ii) the distributed record database that contains information on current machine parameters; (iii) EPICS extensions such as
Alarm Handler, Archiver and MEDM (Motif Editor and Display Manager, a Motif based graphical user interface) well-known to control-room operators; (iv) available Channel Access interfaces to common programming languages (e.g., C, C++, JAVA, TCL/TK or MATLAB).

In the course of SITF operation some of the tools in use approached the end of their lifecycles or became difficult to maintain and will be replaced for SwissFEL. For instance the graphical user interface toolkit MEDM will be replaced by CAQTDM [96], or the scripting language TCL/TK substituted by PYTHON.

Many high-level applications used at the SITF for processing and visualizing information obtained from EPICS channels are written in MATLAB using the MOCHA interface [97], which is based on an in-house C++ Channel Access interface library called CAFE [98]. While this approach enables the quick development of tailor-made measurement programs well suited for a test facility, the resulting applications are typically difficult to maintain. For this reason most of the tools needed for the standard operation of SwissFEL are likely to be reimplemented in PYTHON, again using the CAFE interface [99].

\section{Network structure}

The network layout for the SITF was designed from the start with the SwissFEL requirements in mind. For the SwissFEL facility the required number of network ports is too large to fit into a standard class $\mathrm{C}$ Ethernet subnet, which is limited to 254 addresses. The SITF was therefore intended to be a testbed for a setup integrating several such networks in one facility, although in this case one network would have been enough to cover all addresses. In comparison to a class B network, the advantages of a class $\mathrm{C}$ network are its increased security, scalability and easier error isolation, among other benefits.

The initial SITF network consisted of three class C subnetworks, where the connections between the subnets were controlled by Channel Access Gateways [100] to minimize network traffic. Unfortunately, however, this solution did not perform as expected. The Channel Access Gateways turned out to be bottlenecks that would hamper even normal IOC operation due to bandwidth limitations. The problem was most severe for long waveforms transmitted at high rates. Owing to the many operational disturbances the Channel Access Gateways were removed in early 2013. From then on, directed broadcasts were used to reach EPICS records in the different subnetworks.

\section{Input/output controllers}

To profit from existing know-how and to economize on stock keeping the hardware platforms used as IOCs at the SITF were, as far as possible, selected from the range of platforms already employed at PSI. The following IOC hardware solutions are installed at the SITF: (i) VME64x 
running with the VxWorks operating system (Wind River), mainly using the Motorola MVME5100 card as CPU. A rich set of modules, e.g., for digital and analogue $\mathrm{I} / \mathrm{O}$, are supported. (ii) Motor drivers developed at PSI, using the MAXv-8000 VME controller (Pro-Dex). (iii) Digital power supply controllers developed at PSI. (iv) Programmable logic controllers (PLCs), used for I/O and applications that require high reliability but only moderate speed. The PLCs are based on Siemens S7 series controllers. Typical applications are vacuum interlock and other component protection systems. (v) So-called microIOCs (Cosylab) were successfully tested for the gun laser system. They will, however, not be used further for SwissFEL, since the corresponding product line has been discontinued.

To optimize the management of spare components, the VME hardware for the SITF is housed in 21-slot VME crates of the same type as used for the SLS. The linear, relatively sparse arrangement of the SITF devices, however, resulted in many idle slots. For this reason, smaller 7-slot VME crates have been designed and developed in view of SwissFEL. The new crates in addition provide an easy-touse crate monitoring system, which is interfaced to EPICS. Both old and new crates are manufactured by Trenew Electronic AG.

Ethernet is used as a fieldbus substitute for devices that do not require tight timing synchronization. These devices are read out through so-called Soft IOCs running on central servers.

Special requirements by the diagnostics and llrf systems suggested the use of FPGA-based solutions (see Secs. IV F 2 and IV D 1, respectively). These led to the inhouse development of new boards for SwissFEL: the GPAC (Generic PSI ADC Carrier) for diagnostics [101] and, in collaboration with IOxOS Technologies, the IFC 1210 for llrf and as a future VME CPU board, running embedded Linux as operating system [102].

\section{Configuration management}

Software distribution and installation for the SITF follow the procedures established for other accelerators at PSI [103]. The adopted system facilitates the reproducible deployment of software to the IOCs using a repository based on the Concurrent Versions System (CVS) and a relational database that contains all essential information such as operating system or EPICS versions. The IOC configuration files and the necessary drivers are deployed to a central location, from where the IOCs load them during boot time.

In comparison to, e.g., the SLS, the SITF uses a broad range of IOC hardware, which necessitated a few adaptations of the distribution and installation system to deal with multiple versions of drivers and configurations for several hardware platforms and operating systems. In view of SwissFEL, the integration of Windows-based systems (mainly camera drivers) turns out to be of particular importance.

\section{Timing and event system}

At a pulsed machine such as the SITF the synchronization of different components and control devices at the pulse-by-pulse level is of crucial importance. The synchronization of the various SITF elements is ensured by an MRF timing and event system (Micro-Research Finland Oy). An external reference clock of $124.913 \mathrm{MHz}$ (the 12th subharmonic of the local reference frequency of 1 $498.956 \mathrm{MHz}$ ) and the $50 \mathrm{~Hz}$ mains supply synchronize an event generator. Event information is broadcast in encoded form via optical fibers to the event receivers. The data is decoded by the receivers and appropriate output signals for triggering external devices or interrupts are generated. Further details can be found in Ref. [104].

For the characterization of individual bunches measurements of beam parameters need to be synchronized with the electron bunch and tagged with a unique bunch identification. To this end the timing system supplies a monotonically increasing bunch number consisting of 52 bits. This allows for $2^{52} \approx 4.5 \times 10^{15}$ bunch numbers, more than enough for the lifetime of the facility.

The bunch-synchronized recording of beam data across multiple IOCs was made possible through the development of a so-called beam-synchronous data acquisition (BSDAQ) mechanism [105] using the same event system. In this scheme the IOCs hold the data to be recorded synchronously in local buffers, which are read out for analysis at the end of a predefined acquisition period. A BS-DAQ run can be configured dynamically by the user via the specification of parameters such as the number and spacing of acquisitions and then be initiated and controlled through a graphical user interface. The BS-DAQ application supports the acquisition of waveforms and simultaneous operation by several users.

At SwissFEL the presence of more data sources, the higher event rates and the peculiar mix of rf frequencies [106] call for an upgrade of the timing and event system. The upgrade includes an efficiency enhancement of the BSDAQ system through the implementation of the buffering on a dedicated fast file system.

\section{H. Timing and synchronization}

The tight tolerances on $\mathrm{rf}$ phase stability at FELs represent a major challenge for the distribution of the $\mathrm{rf}$ frequencies along the accelerator. At the SITF, two distribution systems were installed in parallel, an electrical system as the baseline and an optical system using fiberoptic links as a test bed for the SwissFEL implementation.

\section{Reference generation and distribution}

The timing reference generation and distribution at the SITF is based on an electrical master oscillator working at a fixed repetition rate of $214.1365714 \mathrm{MHz}$, an rf amplifier, a coaxial cable distribution and local phase locked 
oscillators with output frequencies of $1498.956 \mathrm{MHz}$, 2997.912 MHz, and 11991.648 MHz [107]. While this approach offered the fastest way to start with a precise timing signal for the main components of the injector facility, such as the gun lasers, the optical master oscillator (OMO), the timing event generation system and the llrf stations, the system is not optimized for drift performance, needs compensation for attenuation and has a limited bandwidth. Since the electrical distribution of the reference signal was soon found to meet the requirements for operating the SITF entirely, not much effort went into stabilizing its cables (e.g., temperature stabilization). Instead further work focused on the development and test of the optical system planned for the SwissFEL facility.

For the final system at SwissFEL the precise timing reference will be based on a purely optical distribution, with an OMO at a repetition rate of $142.8 \mathrm{MHz}$ serving as reference source. This oscillator will be synchronized to an rf master oscillator to obtain the best jitter performance in the spectral range from $10 \mathrm{~Hz}$ to $10 \mathrm{MHz}$. The optical distribution along the $740 \mathrm{~m}$ long facility will be provided, depending on the performance needs, via highly stabilized $\mathrm{CW}$ or pulsed links or via not specifically stabilized links. Where needed, a cable based subdistribution will be installed close to the client location for transmitting $\mathrm{rf}$ frequencies over short distances. At the SITF, a synchronized OMO at 214.137 MHz was operated for testing and development purposes and for developing and operating the bunch arrival-time monitors (see Sec. V G).

\section{Stabilized optical links}

One of the key advantages of stabilized optical links, CW or pulsed, in view of their application at FELs, is their excellent drift performance. In a CW link the optical carrier is modulated with the rf reference signal from an external master oscillator in a Mach-Zehnder electro-optical modulator. In a pulsed optical link the short pulses from an OMO are transmitted and cross-correlated with the partly backreflected pulses from the remote location. At the remote location the optical pulses can be used for applications such as arrival-time monitoring of the electron bunches, optical synchronization of lasers via two-color optical crosscorrelation, or extraction of required rf frequencies from the optical spectrum. In both types of stabilized optical links, phase and amplitude stabilization loops are present to further minimize drifts.

We designed, built and characterized two prototypes of pulsed optical links, one based on cross-correlation and one with temperature-stabilized receivers. The latter type uses both phase and amplitude stabilization loops and was used for the characterization of the bunch arrival-time monitor. In the light of the increasing commercial availability of pulsed optical links, we decided to not focus on building a series of complete systems ourselves.

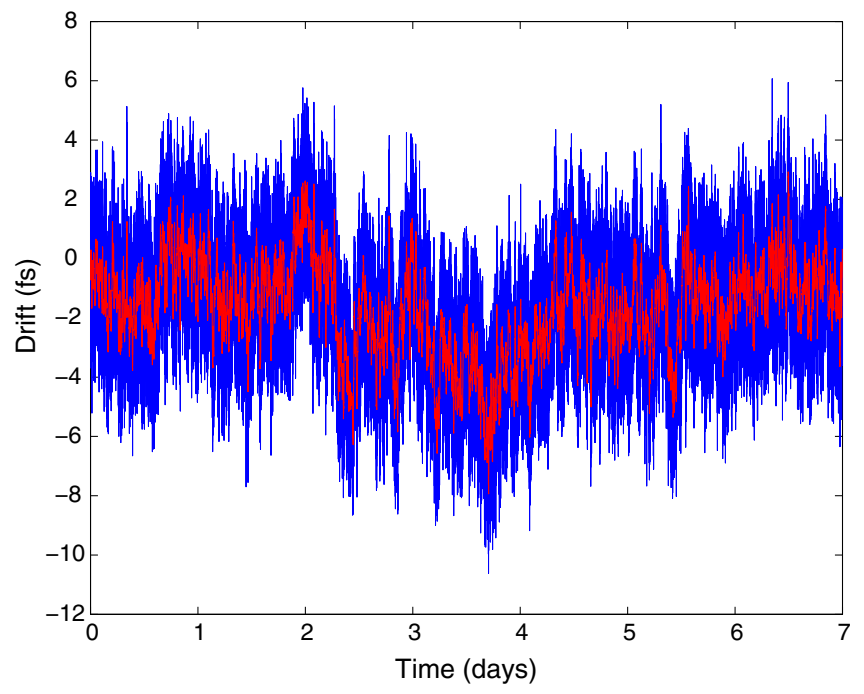

FIG. 35. Long-term drift measurement of a $500 \mathrm{~m}$ long CW optical link: raw data at $1 \mathrm{~Hz}$ (blue curve), and filtered with a time window of five minutes (red curve). See text for details.

We developed and tested CW optical links in-house, in collaboration with Instrumentation Technologies [108]. The CW link system consists of a transmitter and a receiver, which are connected via a pair of optical fibers. The transmitter is fed with an rf frequency $(2997.912 \mathrm{MHz}$ in this case), which is also the output of the receiver. In a linac, the system will transport the rf frequency over long distances, with transmitter and receiver far apart. For the system's characterization, however, they need to be close, because input and output have to be compared with a very sensitive temperature-stabilized phase detector using short rf cables. For the long-term stability and drift measurement presented in Fig. 35, the $500 \mathrm{~m}$ long fiber link was looped back from the transmitter to the nearby receiver. The data were taken at $1 \mathrm{~Hz}$ over seven days (blue curve in Fig. 35). The observed drift values depend on the details of the analysis. For the determination of peak-to-peak values, a well suited method is based on Savitzky-Golay filtering, in our case applied with a third-degree polynomial and a frame size of five minutes, adequate for a measuring time of seven days (red curve in Fig. 35). This results in a peak-topeak drift of 10.9 fs. Shortening the time window to 30 seconds increases the drift to $13.3 \mathrm{fs}$. The rms drift of the raw data amounts to 2.6 fs for the entire seven days.

For those cases where drift performance is not an issue, e.g. the BPM system, not specifically stabilized optical links were developed for cost reasons. The working principle of these links is also based on rf modulation of an optical carrier.

\section{Laser synchronization}

The synchronization of the laser with respect to the gun rf field is of crucial importance for the operation of a 
photoinjector. Also in this case, the synchronization can be achieved by an electrical or an optical system.

At the SITF we developed an electrical synchronization unit for the synchronization of the Nd:YLF gun laser (see Sec. IV A, for which the commercial solution did not fulfill the stringent requirements of photoinjector operation. In addition we successfully tested the optical synchronization by locking the Ti:sapphire oscillator with a $1560 \mathrm{~nm}$ mode-locked erbium-doped fiber laser (EDFL) over a period of 60 minutes.

For SwissFEL, the principal gun laser $\left(\mathrm{Yb}: \mathrm{CaF}_{2}\right)$ will be initially stabilized with an electrical synchronization unit and later on additionally by optical cross-correlation with the pulses from the OMO, which will consist of an erbiumytterbium glass (Er:Yb:glass) laser.

Electrical synchronization of the $\mathrm{Nd}$ :YLF gun laser.-To improve the synchronization of the Nd:YLF gun laser, we implemented our own electrical synchronization unit (phase-locked loop, PLL). It reduced the integrated jitter in the range between $10 \mathrm{~Hz}$ and $10 \mathrm{MHz}$ by an order of magnitude with respect to the original laser synchronization box, down to about 45 fs (see Fig. 36). A better value cannot be achieved since the noise floor of the laser itself in the higher frequency range cannot be reduced due the limited bandwidth of the PLLs of up to several kHz. Further improvements can only be realized during the assembly of the laser.

The described synchronization unit has been integrated into the EPICS control system, allowing for automated locking and tuning via software running on a middle-layer

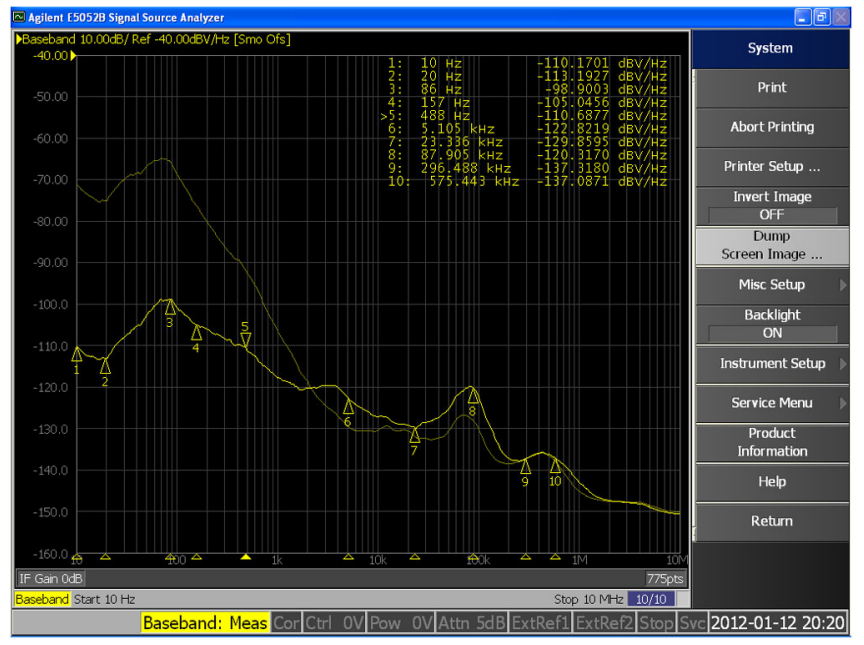

FIG. 36. Signal source analyzer screenshot illustrating the improvement of the jitter of the Nd:YLF laser: Using the inhouse built synchronization unit with gain optimized within the controller bandwidth, the laser exhibits a jitter of $45 \mathrm{fs}$ in the spectral range between $10 \mathrm{~Hz}$ and $10 \mathrm{MHz}$ (bright yellow curve with arrows). For comparison, the original synchronization box results in a jitter of about $600 \mathrm{fs}$ in the same spectral range (pale yellow curve). server. The tuning is necessary to prevent the fine-tuning actuator from leaving its locking range due to changes in the environmental conditions. This delocalized approach for controlling the laser synchronization proved to be very reliable and stable, allowing the laser to stay synchronized with respect to the reference rf for weeks without interruption.

The operation with our synchronization unit results in low jitter values at the oscillator output [36]. Drift measurements of the laser oscillator output including the PLL yield values around 200 fs over 12 hours, depending on the environmental changes and the amplitude-to-phase conversion of the photoreceiver and the phase detector. Since the PLL is not optimized for drift, a further development is under way making use of cross-correlator based optical synchronization. This effort and a proof-of-principle demonstration carried out at the SITF are described in the next subsection.

Optical synchronization of the Ti:sapphire laser.Ultimately, the operation of the SwissFEL user facility will require the synchronization of several laser systems (gun, seed, pump-probe lasers) at a level below $10 \mathrm{fs}$ over hours. Such a degree of stability requires the stabilization of the entire chain from the oscillator to the endpoint, e.g., the photocathode or the experimental probe. An important milestone toward achieving this ultimate goal is the optical locking of two mode-locked oscillators. At the SITF we successfully demonstrated the stability of an optical PLL by using the Ti:sapphire laser oscillator at $800 \mathrm{~nm}$ as a reference to synchronize an EDFL at $1560 \mathrm{~nm}$ [109]. (The Ti: sapphire oscillator was chosen as reference simply to minimize interference with beam operation.) The Ti:sapphire pulses were transported through an approximately $5 \mathrm{~m}$ long free-space transfer line to optically synchronize the EDFL via two-color balanced optical cross-correlation in a $0.5 \mathrm{~mm}$ thick phase-matched BBO crystal. Commercial electronics were used for the locking of the EDFL, which at the time had not been optimized. The results should therefore be regarded as a proof of principle rather than a demonstration of ultimate performance.

A comparison of the phase noise stability of the free running, the rf-locked, and the optically locked EDFL is shown in Fig. 37. The values refer to the absolute jitter including the contribution of the reference. It can be seen that the optical lock gives the lowest noise floor. Normally, the free running and the rf-locked EDFL feature broad noise peaks around $7.5 \mathrm{kHz}$ originating from their controller units. In the case of the rf-locked EDFL, the peak is suppressed by the rf PLL (green trace in Fig. 37). For the optical synchronization, a peak at $5.4 \mathrm{kHz}$ becomes dominant. By tuning the PI gains, this peak can be reduced considerably, albeit at the expense of the stability of the optical lock. With a jitter variation between 80.9 fs and 


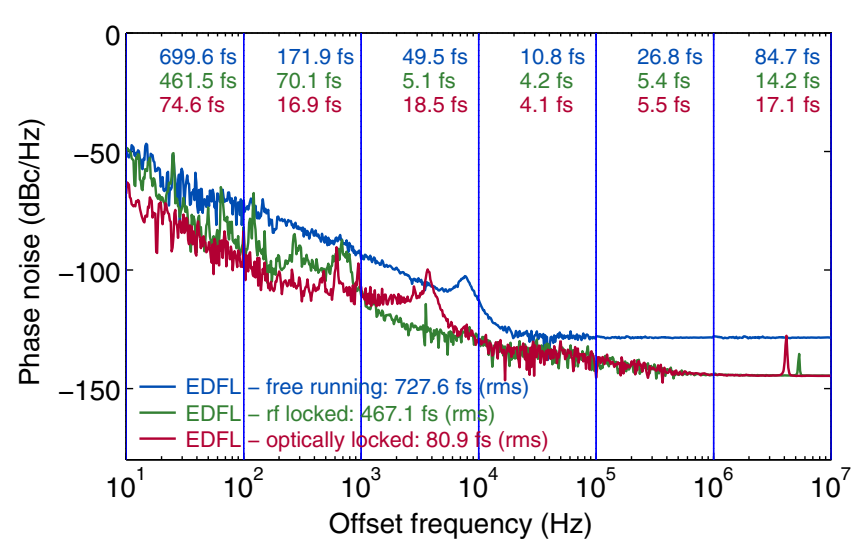

FIG. 37. Phase noise stability comparison between free running, rf-locked, and optically locked EDFL [109]. The values given in the upper part of the figure refer to rms jitters measured in the respective frequency ranges. The values in the lower part of the figure are jitters measured over the entire frequency range (10 $\mathrm{Hz}$ to $10 \mathrm{MHz}$ ).

$217.8 \mathrm{fs}$ (rms) in the range between $10 \mathrm{~Hz}$ and $10 \mathrm{MHz}$ on different days and for different PI gain settings, the optical synchronization provides better stability than the rf one, for which jitter values between 318 fs and $5.6 \mathrm{ps}$ (rms) in the same range have been measured. The wide span of measured jitter values in the case of an rf synchronization comes from the multiple noise spikes in the range between $10 \mathrm{~Hz}$ and $1 \mathrm{kHz}$, which are always present in the phase noise spectra in this setup.

The measured jitter of the optically locked EDFL includes contributions from the phase noise of the PLO, with which the Ti:sapphire is synchronized, and from the transfer line. In the range between $10 \mathrm{~Hz}$ and $1 \mathrm{kHz}$ the optically locked EDFL jitters between 76.5 fs and 118.5 fs (rms), as measured on different days and under different environmental conditions. When the PLO contribution of $75.7 \mathrm{fs}$ (rms) in this frequency range is subtracted, the remaining jitter contribution for the optical loop and the transfer line is between $11.0 \mathrm{fs}$ and $91.2 \mathrm{fs}$ (rms).

The long-term in-loop optical lock stability of the EDFL is shown in Fig. 38. The integrated timing jitter over 60 minutes is $4.6 \mathrm{fs}$ (rms). The ADC sampling rate is 10 samples per second with a bandwidth of $100 \mathrm{kHz}$. Since these are in-loop measurements, they do not incorporate the drifts. Without a zero-crossing feedback on the optical S-curve, the drift will eventually break the optical loop. To test its stability under the influence of an external perturbation, e.g. a drift, an offset voltage is added to the optical error signal and varied manually, see Fig. 39 (left part). The induced changes are followed by the optical PLL within a range of 95 fs (peak-to-peak) before the loop breaks. Figure 39 (right part) illustrates how the optical lock is swiftly reestablished after the removal of the external perturbation and a readjustment of the optical gains.

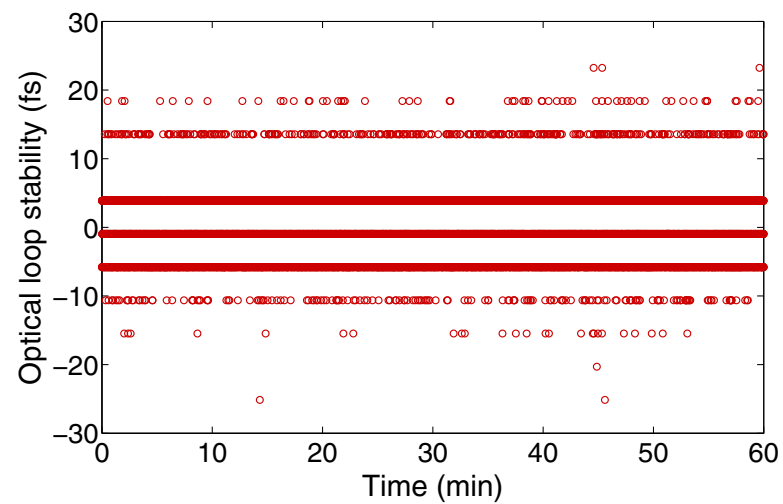

FIG. 38. Long-term in-loop optical lock stability of the EDFL OMO [109]. The integrated timing jitter over 60 minutes is $4.6 \mathrm{fs}$ (rms) in the range between $1 \mathrm{~Hz}$ and $100 \mathrm{kHz}$. The clustering of the stability values at distinct values is a consequence of the limited resolution of the sampling clock.

\section{Superperiod synchronization}

We refer to the greatest common divisor of all $\mathrm{rf}$ frequencies synchronized to a facility's master oscillator as the "superperiod" of that facility. At the SITF the superperiod frequency in use is $2.974 \mathrm{MHz}$. It is distributed via the event timing system, which is synchronized with the master oscillator. The superperiod lock provides an absolute zero reference for all synchronized devices thereby ensuring reproducible timings throughout the machine. The gun laser, for example, with its repetition rate of $83.275 \mathrm{MHz}$, has 18 possibilities to lock to the local reference frequency of $1498.956 \mathrm{MHz}$. Additional

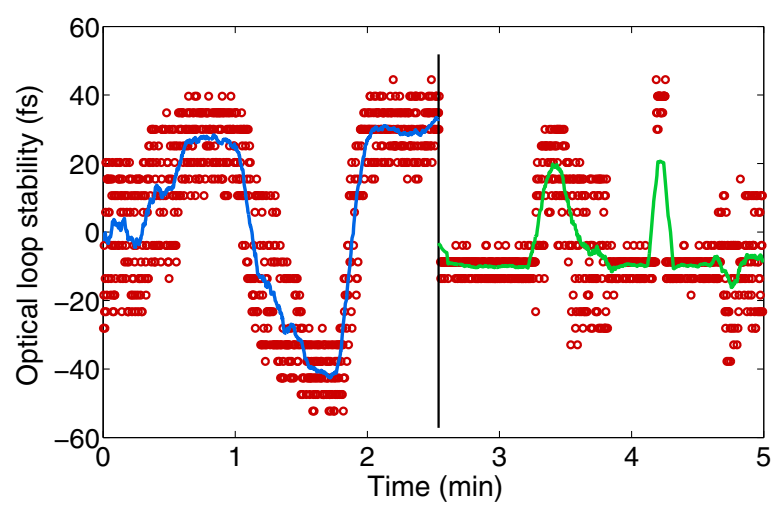

FIG. 39. Test of the stability of the optical phase detector by addition of a manually varied voltage offset to the error signal [109]. The initial curve shows stable operation following the induced changes, until the loop breaks after 2.5 minutes (indicated by the vertical line). The optical lock is recovered once the perturbation is removed and the optical gains are readjusted (curve to the right of the vertical line). The blue and green curves represent sliding averages over 20 consecutive measurements. The integrated timing jitter is $11 \mathrm{fs}$ (rms) in the range between $1 \mathrm{~Hz}$ and $100 \mathrm{kHz}$. (As in Fig. 38, the resolution of the sampling clock leads to a clustering of the stability values at distinct levels.) 
synchronization to the superperiod frequency ensures the same timing of the laser and thus for other components along the machine.

The electronics providing the superperiod synchronization for the Nd: YLF laser, as well as for the SITF OMO, was built in-house. It consists of an rf vector modulator, which shifts the phase of the reference timing until coincidence with the superperiod signal is achieved. The process is automatically steered by a controller board accessible via an RS-232 interface and integrated into the EPICS control system. The superperiod synchronization has performed reliably throughout the operation time of the facility and will be used in a similar way during the initial phase of SwissFEL.

\section{Dose rate monitors}

Dose rate monitors will be required at SwissFEL to prevent radiation-induced demagnetization of the invacuum undulators. At the SITF, the SwissFEL dose rate
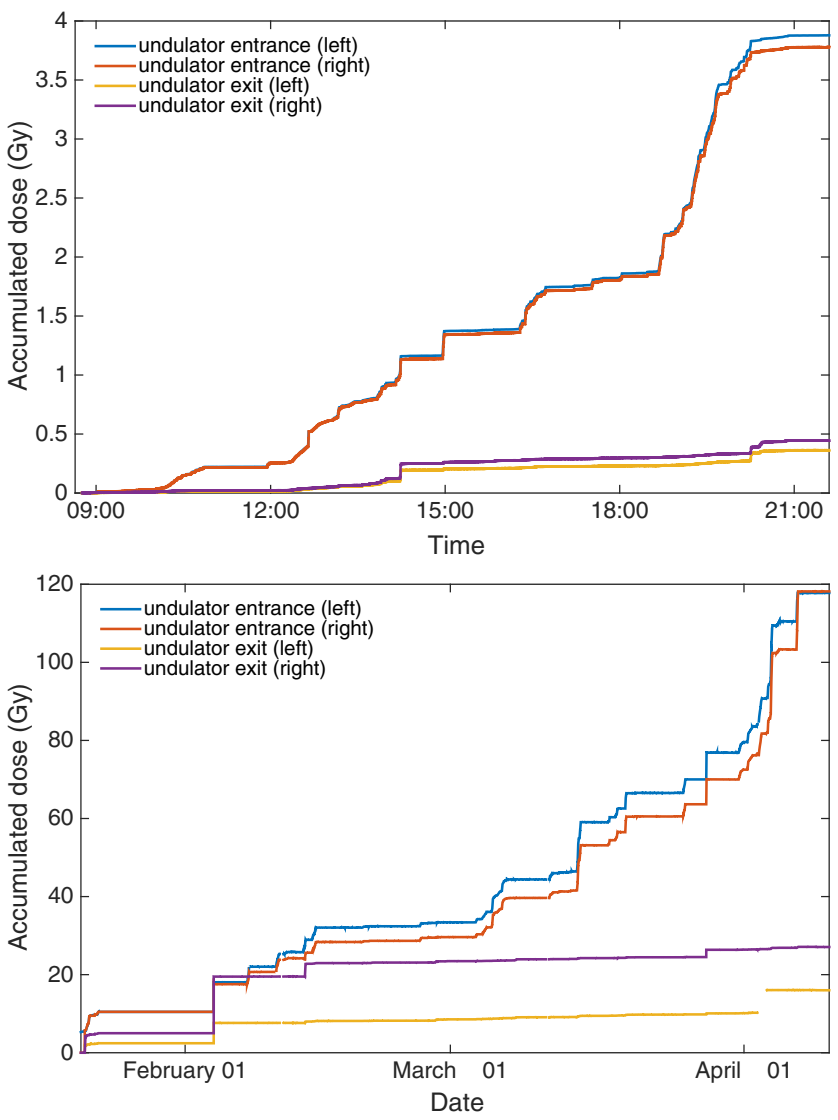

FIG. 40. Top: Snap-shot of the first day of RADFETs monitoring radiation exposure of the undulator. The four curves correspond to the four RADFETs installed at the undulator entrance and exit. Significant doses (of the order of a Gray) can be accumulated in a few minutes, demonstrating the need for a fast interlock. When the machine settings are properly adjusted the remaining losses are moderate. Bottom: RADFETs monitor data for the entire period of the undulator experiment. monitoring system was tested during the phase of the undulator experiment.

In its final state the system will consist of 48 integrating dosimeters based on radiation-sensing field-effect transistors (RADFET) to be placed on each undulator flange, close to the beam pipe, and a few other locations spread throughout the SwissFEL accelerator. The RADFETs are of the type RFT-300-CC10 (REM Oxford Ltd) and are read out by the four-channel dosimetry system DOSFET-L02, developed for FERMI at Elettra [110].

The test at the SITF involved four RADFETs, operated in $25 \mathrm{~V}$ bias mode and each acquiring one reading every 20 seconds. The application of a positive bias significantly increases the response at the expense of limiting the total measurable dose [111]. In this mode, integrated doses of up to 118 Gy could be measured during the approximately 2.5 months of undulator testing. The RADFET readings were cross-checked against the accumulated dose measured with Gafchromic XR-RV3 film dosimeters positioned behind the sensors and found to be in good agreement. Figure 40 shows the dose rate monitored during the first day (top) and the entire duration (bottom) of the undulator experiment.

\section{DIAGNOSTICS DEVELOPMENTS}

In this section we describe diagnostics systems, which were not primarily used for the operation of the SITF, but were utilizing the SITF for tests and developments towards a possible implementation in SwissFEL [112]. An exception is the novel transverse profile imager (Sec. VD 3), which was developed and tested at the SITF, and immediately adopted for routine emittance measurements.

\section{A. Integrating current transformers}

The reference bunch charge measurement at SwissFEL will be provided by integrating current transformers (ICT) $[113,114]$. At the SITF, two types of ICTs have been tested (but were not used for routine operations), Bergoz Instrumentation's standard ICT with BCM-IHR readout electronics [115], and Bergoz Instrumentation's TurboICT-2 with BCM-RF2 readout electronics [116,117].

The standard Bergoz ICT is a calibrated device that measures the total charge in an integration window of $5 \mu \mathrm{s}$. Thus it cannot resolve the two bunches envisaged at SwissFEL. Furthermore it is not immune to dark current, spurious noise of rf, kickers, etc., which introduce additional noise.

The Turbo-ICT-2 is an upgrade of the Bergoz Turbo-ICT featuring absolute charge calibration to within $4 \%$ and twobunch resolving capability with negligible beam position and bunch length dependence. Thanks to its fast readout of the beam induced charge at higher bandwidth it is insensitive to dark current. It also provides usable readings for arrival time jitter less than $1 \mathrm{~ns}$. 


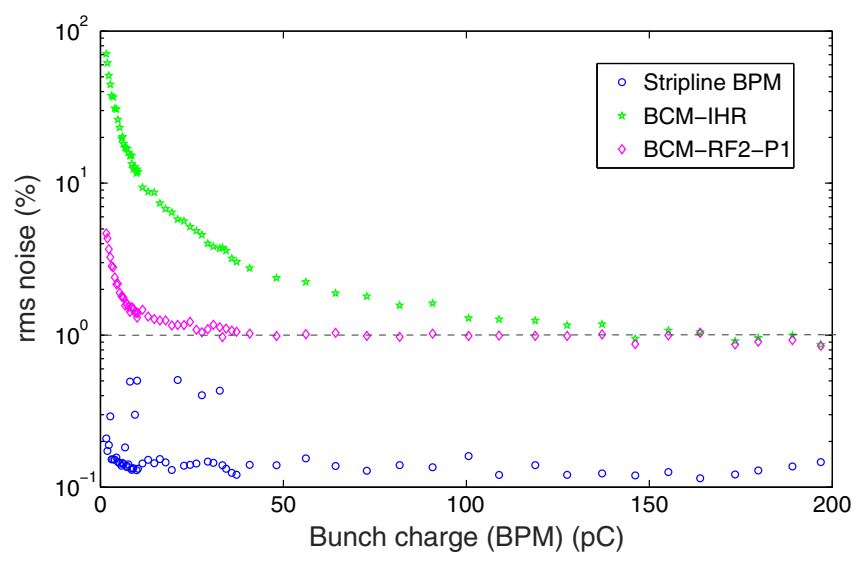

FIG. 41. Noise (rms) measurements of the standard ICT (green stars), Turbo-ICT-2 (first pulse shown, magenta diamonds) and a stripline BPM located close to the ICTs (blue circles), as a function of bunch charge (as measured with a BPM).

We carried out a systematic comparison of the performances of the charge monitors using an uncompressed beam (but using the bunch compression chicane to eliminate dark current) with bunch charge varying between 1 and 200 pC. The rms noise of the charge monitors was determined using the relative charge noise of one stripline BPM with respect to another, and by comparing one stripline BPM to the two ICT monitors. As shown in Fig. 41, the standard ICT has a noise level of about $10 \%$ at $10 \mathrm{pC}$, while the Turbo-ICT-2 is at $1.2 \%$ throughout the nominal bunch charge range between 10 and $200 \mathrm{pC}$, thereby fulfilling the SwissFEL requirements.

The Turbo-ICT-2 was also successfully tested in twobunch mode for a charge range between 1 and $50 \mathrm{pC}$ per single bunch. The measurements showed that the performance for both bunches are within $1 \%$ over the explored charge range.

Considering the requirements for SwissFEL, the TurboICT-2 with BCM-RF2 readout will be used to provide charge measurements and data for beam development and operation. These devices can also be used for BPM crosscalibration throughout the duration of machine operation. A further advantage of the Turbo-ICT-2 is its on-device calibration pulser, which allows for performance selfchecks over long-term beam exposure.

\section{B. Cavity beam position monitors}

While the daily operation of the SITF relied on the resonant stripline beam position monitors (BPMs) described in Sec. IVF2, the development of a more advanced cavity BPM system fulfilling the higher demands for SwissFEL was pursued in parallel [79]. This development included numerous beam tests carried out at the SITF in a dedicated test section near the beam dump of the highenergy spectrometer.

The injector, linac, and beam transfer lines of the SwissFEL accelerator will be equipped with stainless steel
(AISI $316 \mathrm{~N}$ ) cavity BPM pickups with apertures of $38 \mathrm{~mm}$ and $16 \mathrm{~mm}$. These pickups have a nominal frequency of $3.2844 \mathrm{GHz}$, which is well below the beam pipe cutoff frequency for the $38 \mathrm{~mm}$ cavity BPM, and a loaded quality factor $Q_{L} \approx 40$ for the working modes. In two-bunch operation, at the nominal bunch separation of $28 \mathrm{~ns}$, the signal of the first bunch has decayed to $0.07 \%$ of its initial amplitude by the time the second bunch arrives, resulting in very low crosstalk of position and charge readings between the two bunches.

For the SwissFEL undulators, where the BPMs have an aperture of $8 \mathrm{~mm}$, we chose a cavity BPM design with a higher $Q_{L}$ of about 1000 and a working frequency of $4.9266 \mathrm{GHz}$, since these BPMs only need to measure single bunches at $100 \mathrm{~Hz}$ repetition rate. The higher $Q_{L}$ enhances the range-to-resolution ratio for position measurements and simplifies the electronics design described below, while the higher frequency improves the resolution at low charge. In contrast to the $38 \mathrm{~mm}$ and $16 \mathrm{~mm}$ cavity BPMs, the ones for the $8 \mathrm{~mm}$ aperture with their much higher quality factor require copper resonators to reduce resistive losses at the resonator walls to an acceptable level. These resonators are brazed into an outer hull of stainless steel that includes the vacuum flanges. The brazing is done before the machining of the resonators, which is cost-effective and avoids known issues arising from alternative designs based on thin copper coating of stainless steel resonators.

All three SwissFEL cavity BPM pickup types have two resonators: The reference resonator measures the bunch charge via the $\mathrm{TM}_{010}$ mode, while the position resonator measures the product of position and charge via the $\mathrm{TM}_{110}$ mode. The position resonator is equipped with modeselecting waveguide couplers to reject undesired modes. Both resonators have the same frequency and loaded quality factor. Combined with a symmetric design of position and charge signal channels of the BPM electronics, temperature-induced drifts of the beam position readings (where the drift depends on the signal frequency) cancel out to first order when calculating the beam position from the ratio of position and reference cavity signal amplitudes. In addition, critical parts of the cavity BPM electronics are actively temperature stabilized to minimize drift effects in the presence of ambient temperature changes.

The performance requirements for the injector, linac, and transfer line BPMs could also have been met by resonant or nonresonant stripline BPMs. The choice of cavity BPMs for the undulators was clear, however, as these can most easily fulfill the required sub-micron resolution and drift limit for undulator beams that are typically near the center of the beam pipe. In addition, as it turned out, the cavity BPM pickups developed for SwissFEL cost less than the resonant stripline BPM pickups made for the SITF while providing sub-micron resolution and drift at higher bunch charges [79]. The decision to use only cavity BPMs for the 
entire SwissFEL accelerator was therefore a straightforward one.

While sub-micron resolution and drift are mandatory for the undulator BPMs to ensure the alignment and stabilization of the beam trajectory and to optimize electron-photon overlap, it is also highly beneficial for the larger apertures in the accelerator sections upstream of the undulator. The better performance will enable, for instance, highresolution measurements of the beam energy jitter in dispersive sections, where the BPMs will be used as beam energy monitors with an expected relative resolution of $10^{-5}$. It will also allow for the optimization of the FEL photon intensity based on correlations of beam trajectory or energy variations with FEL pulse energy during normal FEL user operation.

Beam tests performed at the SITF involved prototypes of all three cavity BPM pickup types and a preseries version of the readout electronics for the $38 \mathrm{~mm}$ and $16 \mathrm{~mm}$ pickups. Figure 42 shows a simplified schematic of this electronics [118]. For the $16 \mathrm{~mm}$ cavity BPM, a position resolution of $<0.8 \mu \mathrm{m}$ was measured for a bunch charge of $135 \mathrm{pC}$ (with $0.35 \mathrm{~mm}$ beam offset and $>2 \mathrm{~mm}$ peak-to-peak range). The position resolution is determined by correlating the position readings of adjacent BPMs [79].

A first prototype of the $8 \mathrm{~mm}$ cavity BPM electronics was designed and successfully tested, reaching, e.g., $310 \mathrm{~nm}$ rms position noise down to $10 \mathrm{pC}$ charge and within a $1.3 \mathrm{~mm}$ measurement range.

The design is similar to the low-Q electronics for the larger apertures, but has only one mixer per channel, where the three pickup signals are mixed to an intermediate frequency (IF) of about $130 \mathrm{MHz}$ rather than to baseband, thus reducing the number of required ADC channels per BPM from six to three. Beam position and charge are then determined digitally by the FPGA from the undersampled IF waveform. A prototype version of the $8 \mathrm{~mm}$ cavity BPM pickup was tested successfully with beam at the SITF

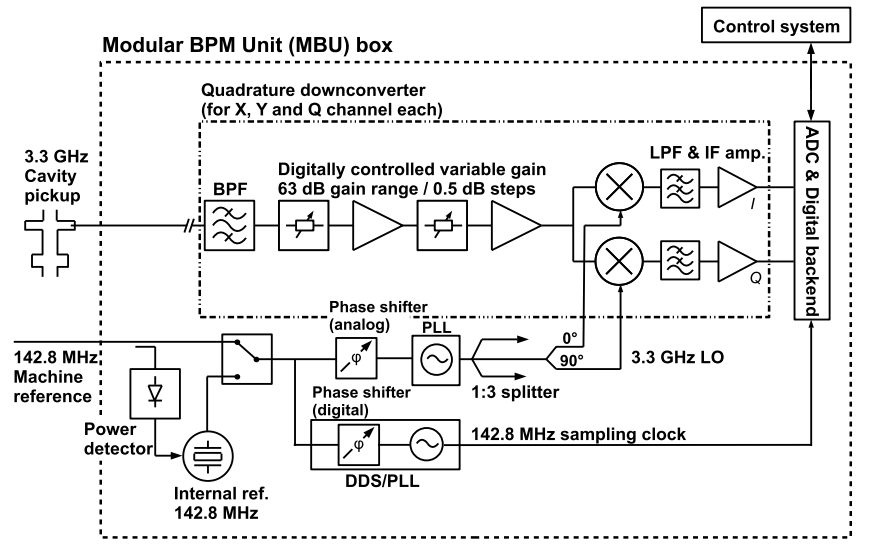

FIG. 42. Simplified schematic of low-Q cavity BPM rf frontend electronics, showing only one of its three input channels (one reference and two position signal channels) [79].
[119]. After the decommissioning of the SITF, the final BPM system is currently being tested with beam at the injection linac of the SLS.

In addition to high-resolution position measurements, all cavity BPMs will also be used for beam charge measurements. The signal scaling factor will be precalibrated in the lab, with an expected scaling factor error of less than $10 \%$. A more accurate beam-based calibration will be performed in situ comparing the readings to those of dedicated charge monitors such as integrating current transformers (see Sec. VA). During beam tests at the SITF with the lowQ cavity BPMs, we typically measured a relative charge resolution of $0.07 \%$ (rms) for high bunch charges, where the absolute resolution is proportional to the charge, and an absolute charge resolution of $8 \mathrm{fC}(\mathrm{rms})$ at very low charges, where the absolute resolution is charge independent. The measured values exceed the SwissFEL specifications of $0.1 \%$ and $30 \mathrm{fC}$, respectively.

\section{X-band wakefield monitors}

The small aperture of the X-band structure may give rise to strong short-range transverse wakefields induced by bunches passing through with a transverse offset. At the relatively moderate beam energies of the SITF the induced wakefields may significantly deteriorate the beam emittance and must therefore be reduced to a minimum by a proper alignment of the structure. Since the mechanical axis of the structure does not necessarily coincide with the electrical axis producing the smallest wakefields, a beambased alignment is essential. Besides the evaluation of the beam emittance downstream of the structure as a function of its position and orientation, a direct measurement of the wakefields via their coupling to the higher-order modes (HOM) of the structure may be used as guidance for the structure alignment.

The X-band structure installed in the SITF is equipped with two HOM couplers, one upstream and one downstream of the structure, designed to be used as wakefield monitors $[57,120]$. The couplers are special cells coupling to the transverse higher order modes in the structure (see Fig. 43). The main reason for using two couplers at either end of the structure lies in the constant-gradient design of the X-band structure, in which the smooth variation of the cell dimensions along the structure compensates for internal losses and keeps the gradient of the fundamental mode constant. This leads to a spread of the synchronous frequency of the position-dependent dipole modes in the 15 to $16 \mathrm{GHz}$ range, i.e., an offset beam will excite lower dipole-mode frequencies upstream than downstream. These modes are typically trapped, their fields extending only over parts of the structure. Two couplers are required to cover the full range of modes.

The correlation between signal frequency and cell number along the structure can be exploited to diagnose higher-order misalignments. For instance, if the structure is 


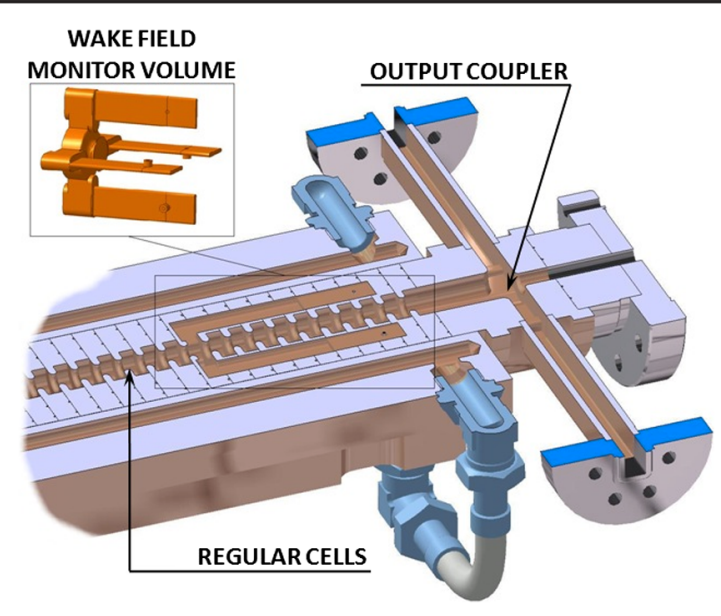

FIG. 43. Cutoff view of the downstream end of the X-band structure showing the location and geometry of the wakefield monitor coupling cell relative to adjacent regular cells, highpower output coupler and cooling circuit [58].

tilted or bent, the offset between the cells and the beam varies along the length of the structure, resulting in a distinct spectral signature. An example of such a measurement is shown in Fig. 44, where the structure was intentionally tilted and the spectrum of the upstream pickup was measured for various offsets. The signal amplitude near $15.3 \mathrm{GHz}$, corresponding to the upstream end of the structure, shows a minimum at roughly $-40 \mu \mathrm{m}$, whereas the center of the structure, probed at a frequency of 15.67 $\mathrm{GHz}$, has an offset of $-140 \mu \mathrm{m}$. Tracing the minimum signal versus the frequency clearly reveals the tilt of the structure.

The clean measurement of the HOM spectrum at frequencies between 15 and $16 \mathrm{GHz}$ in a radiation prone environment represents a technical challenge. In the framework of the EuCARD-2 project [121], a front end based on electro-optical techniques, promising significant advantages in bandwidth, sensitivity, and radiation hardness, is currently under development at PSI [122].

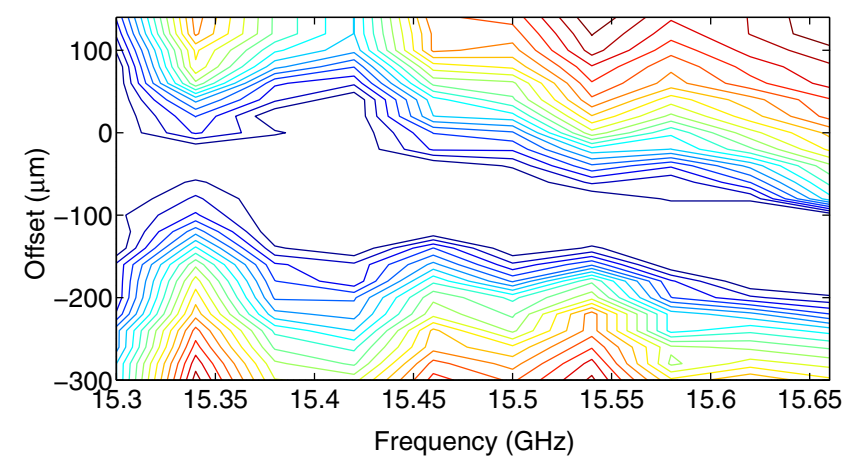

FIG. 44. Signal output measured by the upstream wakefield monitor at different frequencies for a varying structure offset. The structure is tilted on purpose by an angle of $0.5 \mathrm{mrad}$.
In the SwissFEL injector, two X-band structures will be used to linearize the beam's longitudinal phase space at an energy of $320 \mathrm{MeV}$. Both structures will be mounted on motorized support structures and equipped with wakefield monitors. While the associated readout electronics will not be part of the initial project phase, their implementation is envisaged in an early upgrade of the system.

\section{Transverse profile monitors}

Most of the SITF beam operation relied on screens (Secs. IV F 3 and V D 3) for beam profile measurements. In addition to these invasive monitors, we tested and operated minimally invasive and completely noninvasive transverse profile monitors at the SITF: wire scanners and synchrotron radiation monitors, respectively. We describe these systems, and the experience gained with them, in the following two subsections.

\section{Wire scanners}

At SwissFEL, wire scanners are intended to complement the screens in various aspects: First, they provide a quasinondestructive monitoring of the transverse profile of the electron beam during FEL operations. Second, they can resolve the $28 \mathrm{~ns}$ time structure of the two bunches at $100 \mathrm{~Hz}$. And third, they offer an alternative profile measurement in case the imaging performance of the screens is degraded by coherent transition radiation or other effects.

In a wire scanner measurement, a precise reconstruction of the single-axis projection of the beam transverse profile requires the beam-synchronous acquisition (see Sec. IV G 5 and Ref. [105]) of the readout of a beam-loss monitor picking up the electromagnetic shower produced by the wire scanning the beam and the encoder readout of the wire position, together with the charge and position measurements of adjacent beam-position monitors.

Initial tests of the wire scanner system at the SITF were performed with the wires integrated in the screen monitor devices (Sec. IV F 3), where the charge losses were recorded with BPMs. Later studies focused on the final SwissFEL design, for which a prototype installation was integrated into the SITF in July 2014. In this design the wire induced radiation losses are measured by means of scintillator fibers (polymethyl methacrylate, PMMA) wound around the beam pipe and directly connected to a photomultiplier [123]. Figure 45 shows a drawing of the wire fork as it was installed in the SITF beam pipe.

To finalize the design of the SwissFEL wire scanners, a prototype was experimentally characterized on a test bench. The tests included the verification of the stability of the invacuum feed-through of the wire-fork under a steppermotor driver and the measurement of the induced vibrations of the wire stretched on the fork. The measured wire vibration was about $1 \mu \mathrm{m}$, less than the expected scanning 


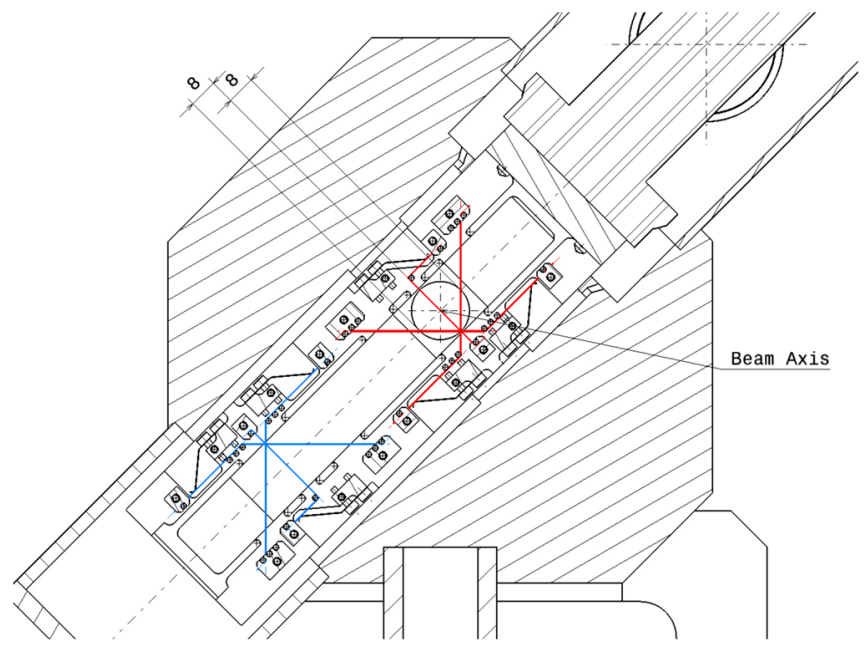

FIG. 45. Front-view of the wire scanner vacuum chamber and the wire-fork. The wire-fork is equipped with 3 different pin slots where $5 \mu \mathrm{m}$ tungsten wires can be stretched (indicated as red and blue lines). The distance between the "wire vertex" and the vacuum-chamber center can be set to $8,5.5$ and $3 \mathrm{~mm}$ corresponding to the 3 different pin slots.

resolution of the wire for the scanning velocities foreseen at SwissFEL [123].

During beam tests at the SITF, several issues concerning the functionality and reliability of the overall system were addressed, such as the beam-synchronous readout of the encoders and the loss monitor, the optimization of the loss monitor signal versus the distance between wire and loss monitor, and the loss monitor sensitivity at low bunch charges scanned by very thin wires ( $5 \mu \mathrm{m}$ diameter).

Recent experimental tests of a prototype of the SwissFEL wire scanner at the FERMI@Elettra FEL facility demonstrated the viability of the SwissFEL wire scanner design also at the GeV energy scale [124].

\section{Synchrotron-radiation monitor}

The deflection of the electron beam in magnetic chicanes offers the opportunity to monitor the beam in a nondestructive way by observing the synchrotron radiation emitted in one of the dipole magnets of the chicane. At the SITF, a prototype setup of such a synchrotron radiation monitor (SRM) using the light emitted by the electron beam at the entrance of the third dipole magnet of the bunch compressor was commissioned and operated. If the chicane dispersion is known, the horizontal projection of the synchrotron light spot allows the determination of the energy spread of the beam induced by the off-crest rf operation.

The setup consists of a two-mirror periscope imaging the synchrotron light from the third dipole magnet to a camera and lens system. Within a range of $\pm 1^{\circ}$ around the nominal bunch compressor bending angle $\left(4.07^{\circ}\right)$ the beam profile of the electron beam can be monitored continuously and nondestructively over the entire range of compression factors $[125,126]$. The sCMOS camera (pco.edge) provides 100 frames per second and, in conjunction with a $300 \mathrm{~mm}$ lens, can resolve the beam profile with a projected pixel size of $0.04 \mathrm{~mm}$. An image analysis algorithm running directly on the camera server can process the images at a rate faster than $100 \mathrm{~Hz}$, providing beam position and size information to the control system for possible use in feedback systems.

Measurements of the beam energy spread performed with compressed beams confirmed that the SRM sensitivity is sufficient to monitor the light spot from a $10 \mathrm{pC}$ bunch over the entire compression range, see Fig. 46.
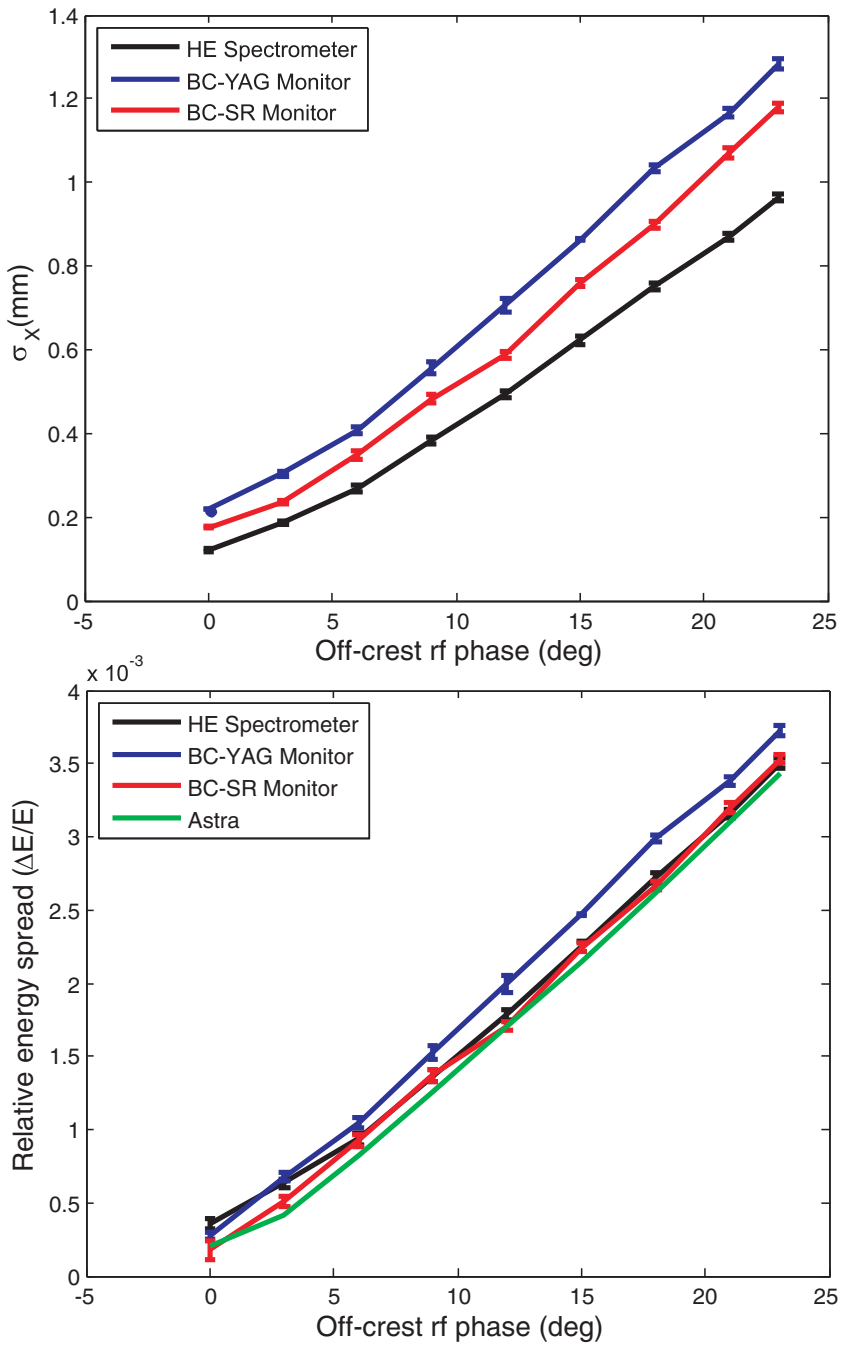

FIG. 46. Top: Horizontal beam size versus off-crest rf phase as measured with the high-energy spectrometer, the YAG screen in the bunch compressor (BC-YAG), and the synchrotron radiation monitor in the bunch compressor (BC-SR) for a beam energy of $230 \mathrm{MeV}$ and a bunch charge of $12 \mathrm{pC}$. Bottom: Relative energy spreads derived from the measurements, compared to the result of a numerical simulation based on ASTRA [127]. 


\section{Novel transverse profile imager}

The limitations of the original transverse profile monitors installed at the SITF mentioned in Sec. IV F 3 called for a redesign of the screen geometry. The new design takes into account both the Snell-Descartes law of refraction and the Scheimpflug imaging condition [128]. In this geometry, (coherent) transition radiation is directed away from the camera, and the scintillating line inside the volume of the crystal is imaged onto a single point, thereby achieving a beam size resolution much better than the crystal thickness. The Scheimpflug imaging ensures focusing over the entire field of view.

A prototype of this novel screen monitor including a high-resolution readout by a CMOS sensor (pco.edge 5.5 camera with global shutter) was installed and tested in the SITF. Cooled by a Peltier element, the sensor has sufficient sensitivity not only for the design operating range of 10 to $200 \mathrm{pC}$, but also for charges well below $1 \mathrm{pC}$ as measured in thermal emittance studies (see Sec. VII A). Figure 47 shows an example measurement of a beam with transverse rms beam size of the order of $20 \mu \mathrm{m}$, much smaller than the crystal thickness, in this case $100 \mu \mathrm{m}$ of YAG:Ce scintillator.

To verify the geometric suppression of COTR at higher beam energies (see Sec. IV F 3) the profile imaging setup was also tested at a location after full compression in the LCLS linac, which is notorious for intense COTR [86]. The measurements, performed at a beam energy of $13.1 \mathrm{GeV}$ and with bunches of $20 \mathrm{pC}$ charge, show no significant variation of the light intensity as a function of the bunch compression [129].

At the SITF, the prototype screen with its outstanding resolution was quickly adopted as the standard screen for all beam optics and emittance measurements in the diagnostics section. The imaging principle worked as expected,

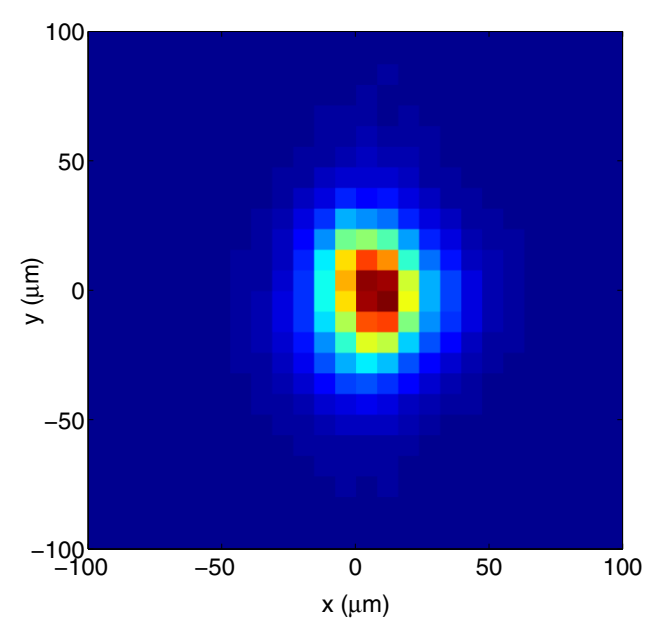

FIG. 47. Example of a beam-size measurement for a bunch charge of $6 \mathrm{pC}$ performed with the novel transverse profile imager. The horizontal and vertical rms beam sizes obtained from Gauss fits are about 15 and $20 \mu \mathrm{m}$, respectively. and only minor revisions to the mechanical design needed to be done for the SwissFEL series production.

\section{E. Coherent-diffraction-radiation based bunch compression monitor}

The electron bunch length is one of the crucial beam parameters determining the FEL performance and thus requires constant monitoring on a single-shot basis. At the SITF we performed preliminary tests of electron bunch compression monitors based on coherent edge and synchrotron radiation emitted at the last dipole magnet of the bunch compressor chicane, see Refs. [130,131] for brief overviews. In this section we focus on the more detailed studies and tests carried out with an electron compression monitor based on coherent diffraction radiation (CDR) generated by the electron bunch when passing through a hole of $6 \mathrm{~mm}$ diameter in a $1 \mu \mathrm{m}$ thick titanium foil (see Fig. 48), located a few meters after the bunch compressor.

The generated spectral density distribution radiated by a bunch is dominated by the longitudinal form factor, defined by the Fourier transform of the longitudinal charge distribution [132]. For rms bunch lengths of 250-500 fs, coherent radiation is expected up to the $\mathrm{THz}$ spectral region. By integrating the spectral intensity of the coherent radiation in a certain frequency range, variations in bunch compression lead to a corresponding variation in the detected intensity.

To increase the sensitivity of the measured signal with respect to small variations in the compression phase, socalled "thick grid" THz high-pass filters are used to select two spectral bands (from 0.26 to $2 \mathrm{THz}$ and from 0.6 to $2 \mathrm{THz}$ ). The focused coherent radiation is subsequently

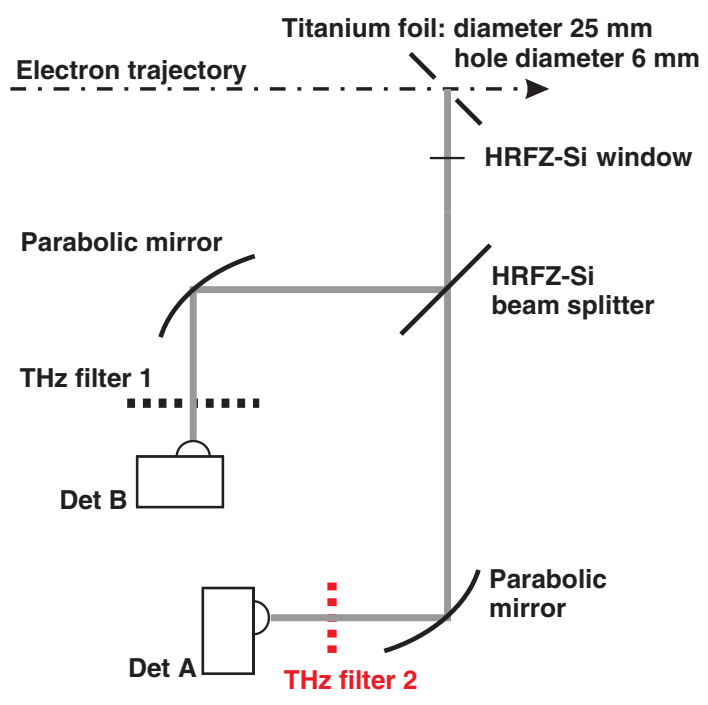

FIG. 48. Schematic setup of the electron bunch length monitor based on coherent diffraction radiation. A window of highresistivity float-zone (HRFZ) silicon is used to transmit the $\mathrm{THz}$ radiation. 


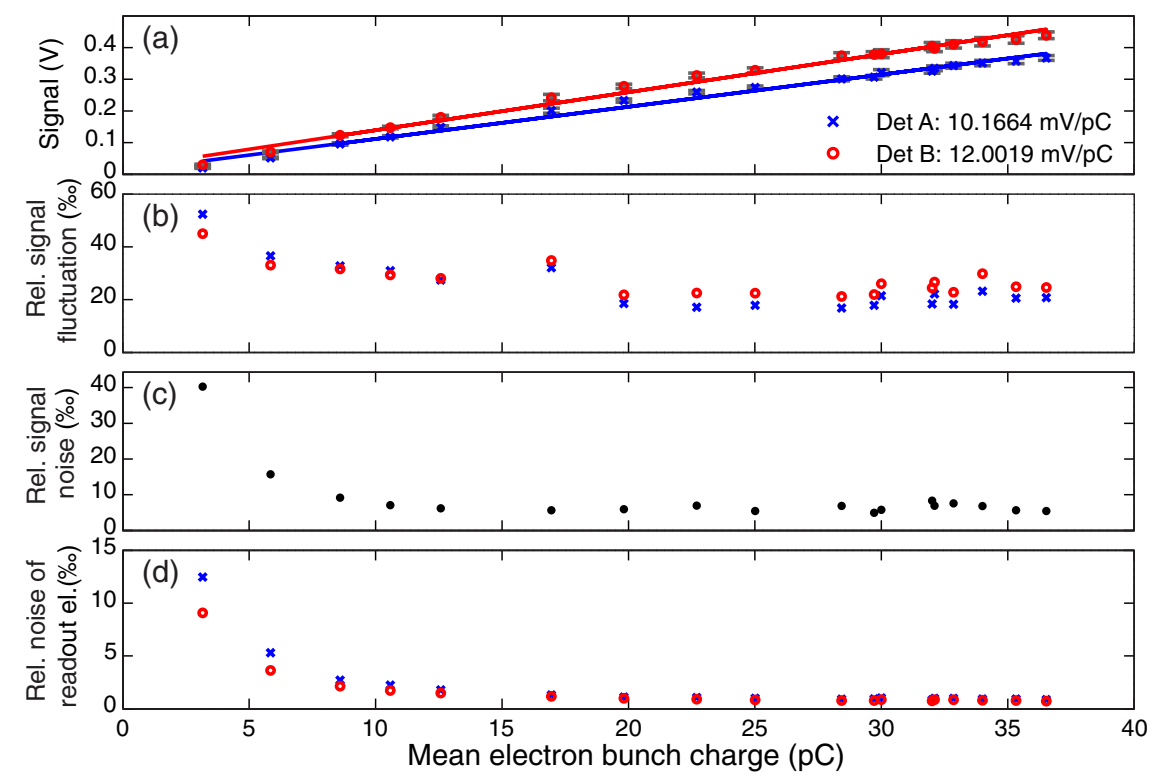

FIG. 49. Detector signals for two identical THz filters (a), relative signal fluctuation (b), detector signal noise as calculated from the correlation from the two detection channels (c), and noise contribution from read-out electronics (d), all shown as a function of the electron bunch charge.

detected by fast Schottky diodes capable of resolving two consecutive bunches separated by 28 ns.

To assess the resolution of the setup, the system was characterized using two identical $\mathrm{THz}$ high-pass filters (both from 0.26 to $2 \mathrm{THz}$ ). In this way, correlated fluctuations can be attributed to changes in the electron beam, while uncorrelated fluctuations are assumed to be caused by detector or readout noise. To characterize the setup at different signal amplitudes, the integrated bunch charge was varied between 2 and 38 pC. Since for large signal levels, the Schottky diode signal is proportional to the electric field strength, we expect a linear dependence on the bunch charge (i.e., the number of electrons) in the case of coherent radiation. This is confirmed by our measurements shown in Fig. 49(a). The slightly different frequency responses of the two detectors give rise to the small discrepancy between the two slopes.

Comparing the relative deviations [Fig. 49(c)] with the noise [Fig. 49(b)] of the detector including cabling and read-out indicates that the main contribution to the overall fluctuations shown in Fig. 49(a) is indeed due to the electron beam. These results were subsequently used to estimate the significance of the measured signals with respect to phase changes of the accelerating if fields as shown in Fig. 50.

The primary application of the compression monitors at SwissFEL will consist in providing a diagnostic signal for the active stabilization of the electron bunch length via control of the relevant rf compression phases. To assess the monitor's suitability for this task, a systematic study was performed, in which the $\mathrm{THz}$ signal was recorded as a function of the common phase of the last two S-band accelerating structures near the nominal compression phase as well as the phase of the X-band structure near the maximum deceleration phase. The results are shown in Fig. 50. For the relatively short electron bunch length of $260 \mathrm{fs}$, a high-pass filter at $0.6 \mathrm{THz}$ clearly enhances the sensitivity. The inferred resolutions in S-band and X-band
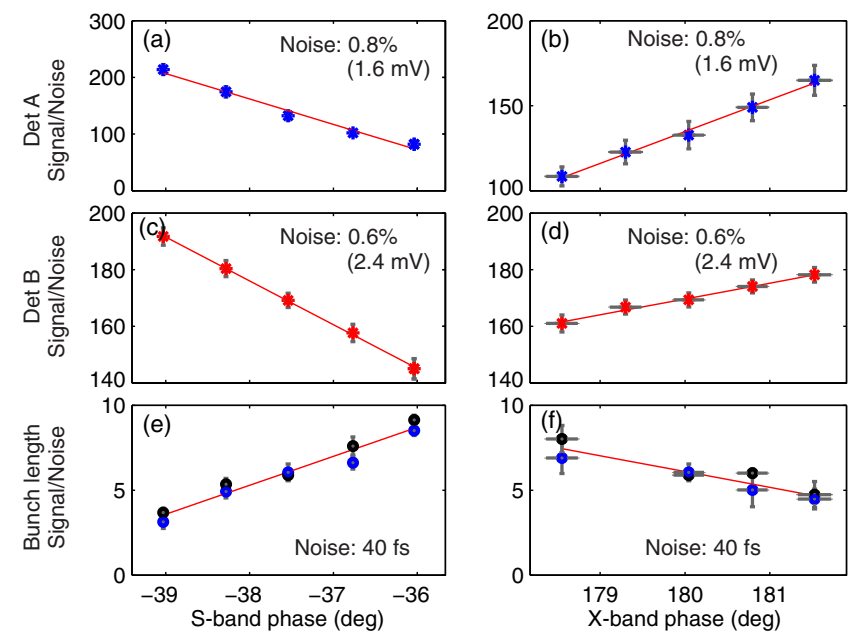

FIG. 50. Detector signals as a function of S-band and X-band $\mathrm{rf}$ phases controlling the bunch compression, shown for two different spectral bands: the signals of detector A, plots (a) and (b), are shown after a high-pass filter at $0.6 \mathrm{THz}$, those of detector $\mathrm{B}$, plots (c) and (d), after a high-pass filter at $0.26 \mathrm{THz}$. For comparison, plots (e) and (f) show corresponding bunch-length measurements performed with the rf deflector. All signal values are displayed in relation to their corresponding noise levels as noted explicitly in the figures. 
TABLE XII. Equivalent resolution in $\mathrm{rf}$ phase achieved by the CDR based electron bunch length monitors at the SITF, in comparison with the resolution goals for SwissFEL.

\begin{tabular}{lcc}
\hline \hline rf band & SITF measurement & SwissFEL requirement \\
\hline S-band & $0.022^{\circ}$ & $0.018^{\circ}$ \\
X-band & $0.054^{\circ}$ & $0.072^{\circ}$ \\
\hline \hline
\end{tabular}

phase along with the stability goals for SwissFEL are summarized in Table XII. A more complete description of the response of the bunch compression monitors, as well as other diagnostics systems in use at the SITF, to rf phase and amplitude variations can be found in Ref. [133].

For SwissFEL, electron bunch compression monitors based on CDR are planned to be installed after the first and the second bunch compressor. Since the operation of the monitors requires the insertion of a foil with a hole into the beam pipe, they will not be entirely parasitic. For this reason, alternative bunch length monitors based on coherent edge and synchrotron radiation from the bunch compressors' last dipole magnets will also be installed at SwissFEL. It is expected that the intensity from coherent edge radiation is higher than the CDR intensity. These monitors, along with a further optimization of the high-pass $\mathrm{THz}$ filter, may allow us to achieve the rf phase resolution goal also in the case of the S-band rf phase.

\section{F. Electro-optic bunch length monitor}

A bunch length monitor based on the electro-optic effect was developed for SwissFEL $[134,135]$ and tested at the SITF [136]. The measurement principle, known as electrooptic spectral decoding, is schematically shown in Fig. 51. A chirped laser pulse, i.e., with time-to-wavelength correlation, is linearly polarized and sent through a $2 \mathrm{~mm}$ thick $\mathrm{GaP}$ crystal installed in the vacuum beam pipe. The laser polarization is changed in the crystal via the electro-optic effect caused by the electric field of the passing electron bunch. A second polarizer after the crystal turns the polarization modulation into an intensity modulation with time-to-wavelength correlation known from a direct calibration procedure. The bunch profile can then be reconstructed from the intensity pattern measured by a grating

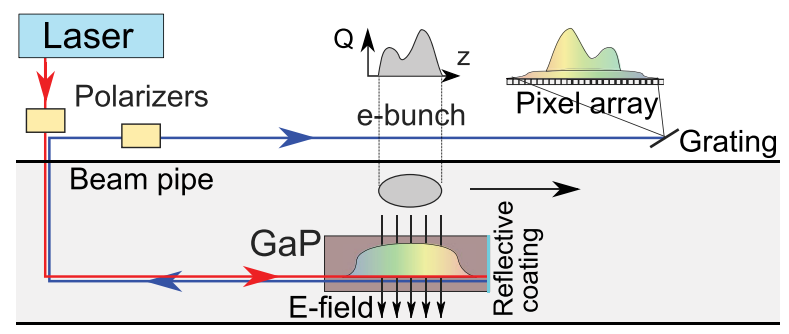

FIG. 51. Schematic representation of the electro-optic bunch length monitor.

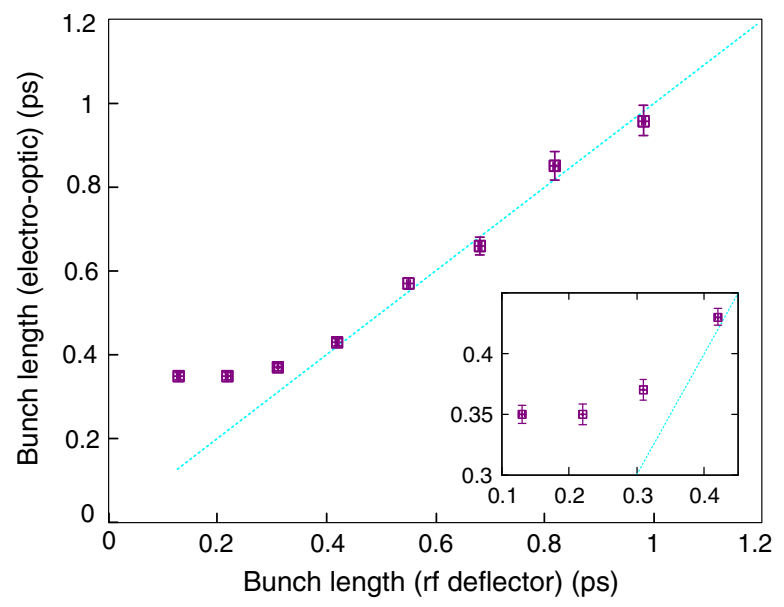

FIG. 52. Comparison of bunch length measurements obtained with the electro-optic monitor and the transverse deflecting rf cavity.

spectrometer. Since the technique is nondestructive it can be used for the online monitoring of the bunch length on a pulse-to-pulse basis at rates up to $200 \mathrm{~Hz}$, restricted only by the spectrometer readout.

One of the studies conducted at the SITF was dedicated to the measurement of the resolution limit of the electrooptic monitor. To this end, electron bunches with $200 \mathrm{MeV}$ energy were compressed in the magnetic chicane and measured under the same conditions with both the electro-optic monitor and the transverse deflecting cavity, which has a resolution well below $100 \mathrm{fs}$ (see Sec. IV F 4). A comparison of the bunch lengths obtained with the two methods is shown in Fig. 52 and indicates saturation of the electro-optic monitor at about 350 fs. The main resolution limiting factor for this method was found to be the frequency mixing effect (see Ref. [135]). For the crystal installed at the SITF, the best resolution that can be expected is around $200 \mathrm{fs}$.

For budget reasons, electro-optic monitors are not included in the current plans for SwissFEL. It is possible, however, that such monitors may be added in a future upgrade of the SwissFEL beam diagnostics system.

\section{G. Bunch arrival-time monitor}

For the monitoring and optimization of the longitudinal linac stability, the SwissFEL concept foresees the nondestructive measurement of the electron bunch arrival time at several locations with resolution better than 10 fs. Two prototype setups of such bunch arrival-time monitors (BAM) have been installed and tested at the SITF, one upstream and, later, one downstream of the bunch compression chicane. To meet the SwissFEL requirement of time resolution below $10 \mathrm{fs}$ in the entire bunch charge range between 10 and $200 \mathrm{pC}$, these prototypes have undergone several iterations toward an optimal use of the component bandwidths and an improved signal-to-noise ratio at low 
bunch charges. In the following we describe the general design of the SwissFEL BAM system and its implementation at the SITF (Sec. V G 1), as well as the evaluation of rf pickups (Sec. V G 2) for SwissFEL.

At the SITF, the BAMs have been used to characterize bunch arrival-time drift and jitter [137], beam energy jitter [138], as well as the longitudinal dispersion (the $R_{56}$ parameter) of the bunch compression chicane. In Sec. V G 3 we give a brief account of the arrival-time drift and jitter measurement. The longitudinal dispersion measurement is described later in the section on bunch compressor characterization (VI A 3). For the beam energy jitter measurement we refer to Ref. [138].

\section{Design and implementation}

The SwissFEL BAM concept is based on a MachZehnder-type electro-optic modulator (EOM) [139], which is interfaced to an optical master oscillator (OMO, see also Sec. IV H 1). The OMO is a phase-locked-loop stabilized mode-locked laser oscillator with a wavelength of $1560 \mathrm{~nm}$ and a repetition rate of $214.137 \mathrm{MHz}$. It delivers reference pulses of a few 100 fs duration to the BAM stations via single-mode optical fiber links, whose lengths are stabilized via optical cross-correlation [140]. This pulsed optical reference is highly stable against drift and jitter, at a level below 10 fs.

An electron bunch passing through the beam pipe at the location of the BAM generates an S-shaped bipolar transient with a steep slope in an rf vacuum pickup in the beam pipe. The pickup signal is transmitted to the rf port of an EOM, which is DC biased in quadrature (i.e., the optical signal is reduced to half its nominal amplitude at the output). These EOMs are part of the electro-optic front-end, which is housed in an enclosing (the "BAM box") close to the beam pipe to avoid bandwidth losses in the rf cables leading to the EOM. The box is temperature stabilized via its base plate at $28^{\circ} \mathrm{C}$ to within $0.01^{\circ} \mathrm{C}$ and shielded against neutrons and X-rays.

Two EOMs are available in the front-end, which provides the flexibility to operate in high-sensitivity as well as high-dynamic-range configurations. The second channel operates at the same zero crossing as the first but uses a separate pair of pickups. Furthermore, the slew rate is filtered for that channel such that the rf slope is less inclined, resulting in a larger dynamic range of the BAM. At the SITF, the second EOM channel was mainly used to simultaneously test different EOMs, rf pickups or other rf components such as attenuators or limiters.

Accommodating the end of the optical fiber link, the BAM box also houses two erbium-doped fiber amplifiers (EDFA) and a Faraday rotating mirror (FRM). The first EDFA, which is part of the optical link and precedes the FRM, serves as an actuator for the link amplitude feedback loop. After the FRM the second EDFA (out of loop) controls the power sent to the BAM photoreceiver (see below).

In the EOM the rf pickup signal is sampled at zero crossing by one of the reference (OMO) laser pulses. The coincidence between a laser pulse and the zero crossing of the rf signal is ensured by setting the laser timing accordingly. Since the OMO laser is locked to the superperiod of the machine reference, this zero-crossing overlap provides an absolute timing reference to any device that is locked to the same superperiod (for instance the gun laser). Any temporal offset due to a change in the electron time of flight leads to an additional positive or negative voltage input at the EOM's rf port, thereby increasing or decreasing the amplitude modulation of the one reference laser pulse which overlaps with the electron bunch. Thus the bunch arrival time is encoded in the amplitude of a single reference laser pulse, while all the other pulses remain unaffected (i.e., premodulated to half amplitude).

The optical pulses from the two BAM channels are received, together with a sampling clock signal, in a data acquisition back-end located in the technical gallery outside the accelerator tunnel. The sampling clock is generated by the same reference pulse train, split in the BAM box before the EOM. The pulses are conditioned in an in-house designed optical photoreceiver module before being readout by a fast 12-bit analog-to-digital converter (ADC) with $500 \mathrm{MHz}$ bandwidth. The data acquisition chain is synchronous to the bunch rate.

The photoreceiver module uses high-bandwidth photodiodes followed by bandpass filters and high-dynamicrange transimpedance amplifiers. The bandpass filters extract either the sine component of the sampling clock or quasi-Gaussian pulses, with 90 ps broad peaks and a few nanoseconds long intervals with a flat baseline in between, from the BAM signals. After the pulse form manipulation, the reference laser pulses are additionally amplified and matched to the ADC input. To ensure the optimal use of the ADC dynamic range in view of a better time resolution, a DAC module (the PSI Analogue Carrier Board, PAC [141]), providing additional DC offset and amplitude control, should be used. (This module only became available for the second BAM station downstream of the bunch compressor.)

The ADC does not sample a waveform, but only the peak or baseline points of a large number of reference laser pulses, most of which precede the pulse that is modulated by the electron bunch. Therefore, a single-shot measurement determines not only the bunch arrival time, but also the error limiting the resolution due to the laser amplitude jitter and the influence of the subsequent opto-electronic chain. In other words the BAM is a self-referenced system capable of measuring its own resolution on a shot-to-shot basis. The BAM resolution is essentially a function of the slew rate of the rf transient and the effective amplitude jitter of the reference laser pulses, as measured by the ADC. For 
our system, the latter effect was found to dominate. The measured effective laser amplitude fluctuations vary between $0.25 \%$ and $0.3 \%$.

Since both the sampling clock and the signal are generated from the same optical pulse train, the ADC sampling position on the reference laser pulse remains unchanged under temporal shifts of the laser reference. Such temporal shifts of the laser are brought about, for instance, during the calibration of the system, when the modulated laser pulse is scanned over the rf transient to map the extent of the amplitude modulation to the laser delay. In a similar vein, a dedicated delay stage in the BAM box is applied to run a zero-crossing feedback: each time the bunch arrival time exceeds a predefined limit determined by the slope of the rf transient, the reference laser pulse is shifted back to the zero crossing, thereby keeping the BAM acquisition within the dynamic range of the transient.

\section{Evaluation of rf pickups}

Two types of rf pickups were evaluated in the first BAM station (upstream of the bunch compressor), a button and a ridge waveguide, both mounted on a common $38 \mathrm{~mm}$ vacuum chamber [137].

The ridge wave guide features a high coupling strength to support measurements at low bunch charges and an intrinsic bandwidth of $16 \mathrm{GHz}$. Measurements with this pickup type revealed a strongly nonuniform bunch charge dependence of the BAM signal with maximum slew rates (resulting in the best BAM resolution) around $100 \mathrm{pC}$ bunch charge and dropping again at higher charges. In view of the limited dynamic range with respect to bunch charge, this design was considered impractical for SwissFEL.

The button pickup is designed as a $50 \Omega$ coaxial line, starting with a cone shaped tip with $2.5 \mathrm{~mm}$ base and smoothly tapered over the $28 \mathrm{~mm}$ length to a coaxial glassbead feedthrough and a $2.92 \mathrm{~mm}$ connector. Its nominal intrinsic bandwidth is $80 \mathrm{MHz}$. Test results obtained with the button pickup show a uniform bunch charge dependence. The slew rates, however, are limited by the coupling strength at low bunch charges and by the feedthrough bandwidth of $20 \mathrm{GHz}$.

Based on this experience, the initial plan for SwissFEL was to increase the coupling strength by shortening the $50 \Omega$ coaxial line to $6 \mathrm{~mm}$ and by extending the bandwidth of the feedthrough to $40 \mathrm{GHz}$. The design diameter of the button remained at $2.5 \mathrm{~mm}$ and is a compromise between signal strength and bandwidth $(40 \mathrm{GHz})$. The production of such vacuum feedthroughs, however, turned out to be a substantial technical challenge. Two approaches were pursued, one based on commercial high-bandwidth boresilicate glass beads and another wherein a kovar glass is directly sintered to the flange. Unfortunately the corresponding prototypes turned out to be both costly and timeconsuming in production and did not meet the design specifications. We therefore decided to abandon this development and started testing two pickup prototypes developed by TU Darmstadt and DESY [142,143]. The second BAM station at the SITF, positioned after the bunch compression chicane is equipped with rf pickups of this type. The arrival time resolutions obtained with this BAM station, using an rf pickup prototype adapted to the SwissFEL $16 \mathrm{~mm}$ vacuum chamber, are $35 \mathrm{fs}$ for a bunch charge of $10 \mathrm{pC}, 20 \mathrm{fs}$ for $20 \mathrm{pC}$ and vary in the range from 13 fs down to 7 fs for bunch charges between $30 \mathrm{pC}$ and $200 \mathrm{pC}$. For the SwissFEL BAM stations in the undulator section, where the beam pipe diameter is only $8 \mathrm{~mm}$, a further improvement in resolution at low bunch charges by about a factor of two is expected.

\section{Arrival time drift and jitter measurement}

An example of a long-term bunch-arrival-time measurement performed with the BAM located upstream of the bunch compressor over approximately eight hours is given in Fig. 53. For this measurement, the Nd:YLF based gun laser system was used, locked to the $1498.956 \mathrm{MHz}$ rf master oscillator and to the machine superperiod. The average bunch charge during the measurement period was $130 \mathrm{pC}$. The optical fiber link was stabilized with an accuracy of 21 fs. During the run the link motor had to compensate up to $47 \mathrm{fs}$ of fiber drift (Fig. 53, lower plot). A feedback ensures that the BAM acquisition is always kept near the zero crossing of the BAM pickup slope. The resolution is $20 \mathrm{fs}$, which was typical for our initial pickup design, where the bandwidth was limited by the vacuum
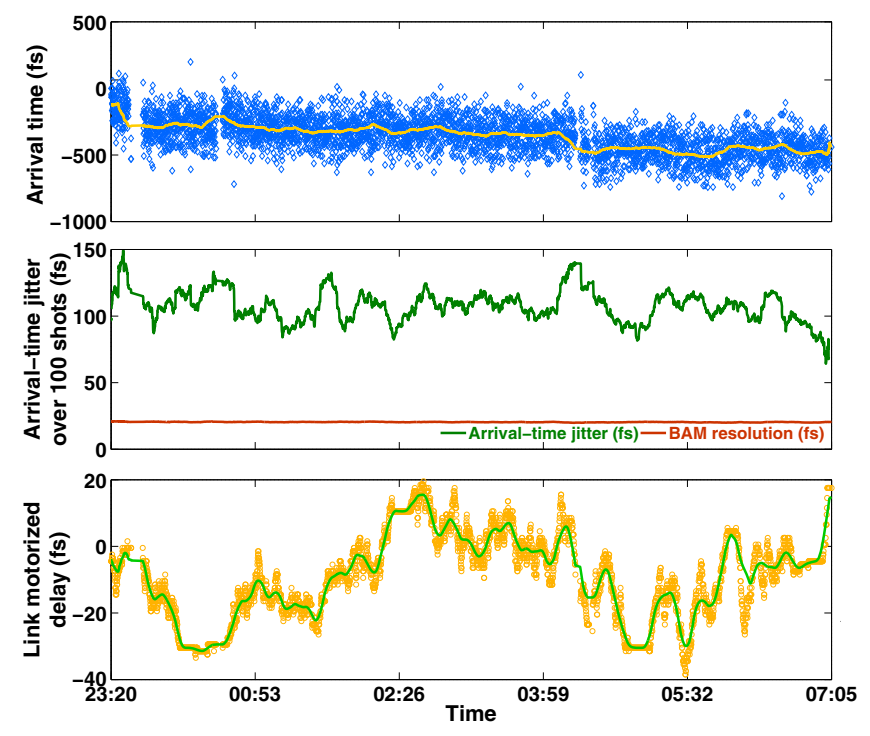

FIG. 53. Long-term bunch arrival-time measurement over a period of eight hours, using the BAM upstream of the bunch compressor at a bunch charge of $130 \mathrm{pC}$ (top), arrival-time jitter over 100 shots (middle) with instantaneous resolution (red curve), and link length compensation applied by the motorized delay line (bottom). 
feedthroughs $(20 \mathrm{GHz})$ and the electro-optic intensity modulators $(12 \mathrm{GHz})$. The bunch arrival-time drift, determined as a sliding average over 100 shots (i.e., approximately $10 \mathrm{~s}$ ), is $410 \mathrm{fs}$ (peak-to-peak) over the 8 hour period. During this period several rf interlocks occurred, leading to beam interruptions visible as data gaps in the drift plot (Fig. 53, upper plot). After recovery of the rf the BAM acquisition was resumed automatically. The middle plot of Fig. 53 shows the jitter, defined as the deviation of the instantaneous arrival time from the smoothed average described above. The jitter ranges between 70 and $150 \mathrm{fs}$, with an rms value of $110 \mathrm{fs}$. Similar jitter values were obtained even before the commissioning of the optical fiber link. A substantial fraction of the observed jitter arises from the gun laser amplifier and transfer lines after the stabilized laser oscillator. A dedicated laser arrival-time monitor is currently under development for SwissFEL with the goal of characterizing and mitigating this effect [144].

\section{BEAM AND LATTICE CHARACTERIZATION PROCEDURES}

In addition to advanced beam diagnostics, the detailed characterization of the electron beam generated at the SITF also relied on elaborate methods and procedures, which first needed to be established at our facility, and later refined and adapted for routine use. In this section, we describe these procedures, before presenting the main results in Sec. VII.

\section{A. Lattice characterization and setup}

The detailed knowledge of the lattice acting on the beam is essential for the proper interpretation of all beam physics measurements. We present our efforts to characterize and adjust the beam orbit in all parts of the machine, including the effects of the bunch-compressor and geomagnetic dipole fields.

\section{Beam-based alignment}

All machine components were mechanically aligned during the installation phase with a precision better than $100 \mu \mathrm{m}$. In addition, a beam-based alignment was performed for those beam-line components that are relevant for beam optics measurements.

For the beam-based alignment of the rf gun elements, we essentially follow the procedures described in Ref. [145]. In a first step, the laser position on the cathode of the electron gun is adjusted to the central axis of the gun rf field. In the case of the CTF gun, this is determined by the center of the dark current halo, as it can be observed on one of the first view screens of the gun section with gun solenoid and laser both turned off. The position of the laser spot on the cathode is then adjusted until the photoelectron beam and the dark current halo are concentric. During the procedure, all corrector dipole magnets between the gun and the view screen are turned off since in general the energy of the dark current electrons differs from that of the photoelectrons. To improve the visibility of the photoelectron beam on the screen, the rf phase is shifted toward the phase of maximum charge extraction, resulting in a focusing of the photoelectron beam by the $\mathrm{rf}$ field, and the $\mathrm{rf}$ gradient is reduced by $10 \%-20 \%$ to increase the contrast between the dark current halo and the photoelectron beam.

The laser position found in first approximation by centering on the dark current halo may be further readjusted by verifying that the beam position on the screen is insensitive to changes of the gun rf phase. In the case of the SwissFEL gun (from April 2014 on), the amount of dark current produced was insufficient for the simple alignment procedure based on the dark current halo and an rf phase scan was needed for the laser alignment.

Once the gun laser spot is fixed, the gun solenoid is aligned by minimizing the movement of the photoelectron beam, again observed on one of the first view screens of the gun section, in response to changes in the strength of the solenoid field. The gun solenoid is mounted on a motorized support with four degrees of freedom (the longitudinal position and the rotation angle around the beam axis are fixed). For a large variation of the solenoid excitation current from 20 to $150 \mathrm{~A}$, a variation of the beam position well below $1 \mathrm{~mm}$, measured on a screen $47 \mathrm{~cm}$ downstream of the solenoid exit, is achieved routinely. The nominal current is around $120 \mathrm{~A}$, corresponding to an integrated field of about $0.006 \mathrm{Tm}$.

All beam-line elements following the gun section, with the exception of the X-band structure, are not motorized and cannot be aligned mechanically by remote control (the bunch compression chicane can only be moved in one direction). Instead the beam reference orbit, i.e., the orbit to be approximated by a manual or automated orbit correction procedure (feedback) is adjusted for optimal beam propagation. In practice this is achieved by adding (or subtracting) offsets to the BPM readout positions before using them for orbit correction. The task of alignment then consists in finding and applying the appropriate BPM offsets in each section.

In the case of the booster section, the reference orbit is adjusted to coincide with the central axis in each accelerating structure. This coincidence is achieved when the beam trajectory remains constant after a change in $\mathrm{rf}$ gradient in the given structure. Figure 54 shows the difference of the beam trajectory before and after the first booster section alignment in the horizontal plane.

Downstream of the booster the reference orbit is chosen to minimize spurious transverse dispersion along the beam line (dispersion-free steering [146]). The amount of spurious dispersion after the booster is determined by observing the orbit deviation in response to a variation in the beam momentum. The beam trajectory (position and angle) at the exit of the S-band section is fixed through an orbit feedback 


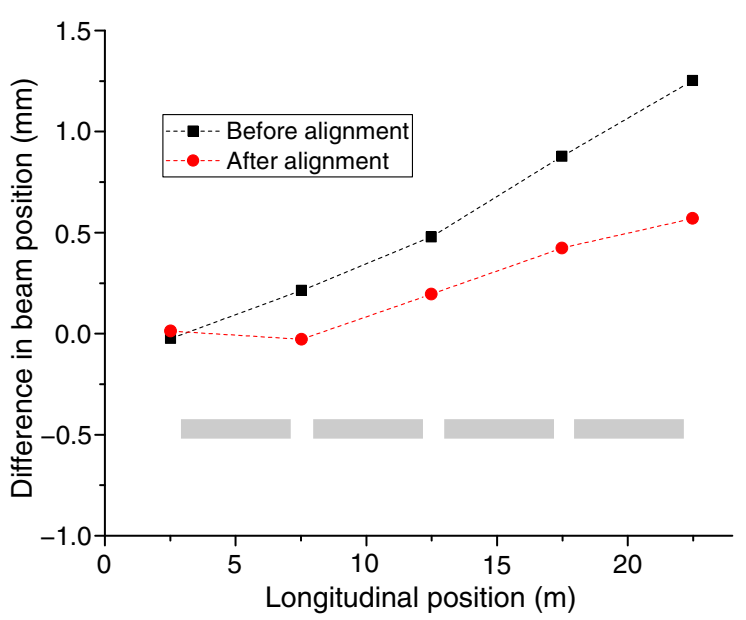

FIG. 54. Measured beam position variation along the injector in the horizontal plane in response to a change in rf gradient, before and after the beam-based alignment of the first booster structure. The beam position at the second BPM immediately after the first S-band structure is almost unchanged after the alignment, whereas further downstream changes persist due to the still misaligned structures. The locations of the four booster structures are indicated as gray boxes.

to compensate possible residual cavity kicks induced by the momentum change, which may still remain after the beambased alignment of the booster structures. The variation in beam momentum is achieved by shifting the second S-band booster structure from on-crest to off-crest acceleration. We vary the phase and not the gradient to avoid possible temperature effects in the structure following a change in gradient ( $r f$ power), which could matter over the timescale of the alignment procedure.

In Fig. 55 we show the measured spurious dispersion before and after adjustment of the reference orbit. While the dispersion is reduced to a satisfactory level, the correction is only valid for the quadrupole setting at the time of the correction. Since the matching quadrupoles in the X-band section are frequently adapted depending on the beam shape at the exit of the S-band section, the BPM offsets need to be updated whenever a significant change of the quadrupole settings has occurred.

The X-band structure, positioned between the booster and the bunch compressor to linearize the longitudinal phase space for optimal compression, can be aligned actively. Similar to the gun solenoid, it is mounted on a remotely controlled movable platform with four degrees of freedom. The position of the structure can be varied within a range of about $\pm 2 \mathrm{~mm}$ in both transverse dimensions. The optimal position and orientation of the structurerelative to the reference orbit as determined by dispersion minimization - are found empirically by minimizing the measured emittance of the uncompressed beam. In addition, the wake field monitors described in Sec. V C may be used to find the optimal orbit through the structure. The two methods are found to agree well within errors.
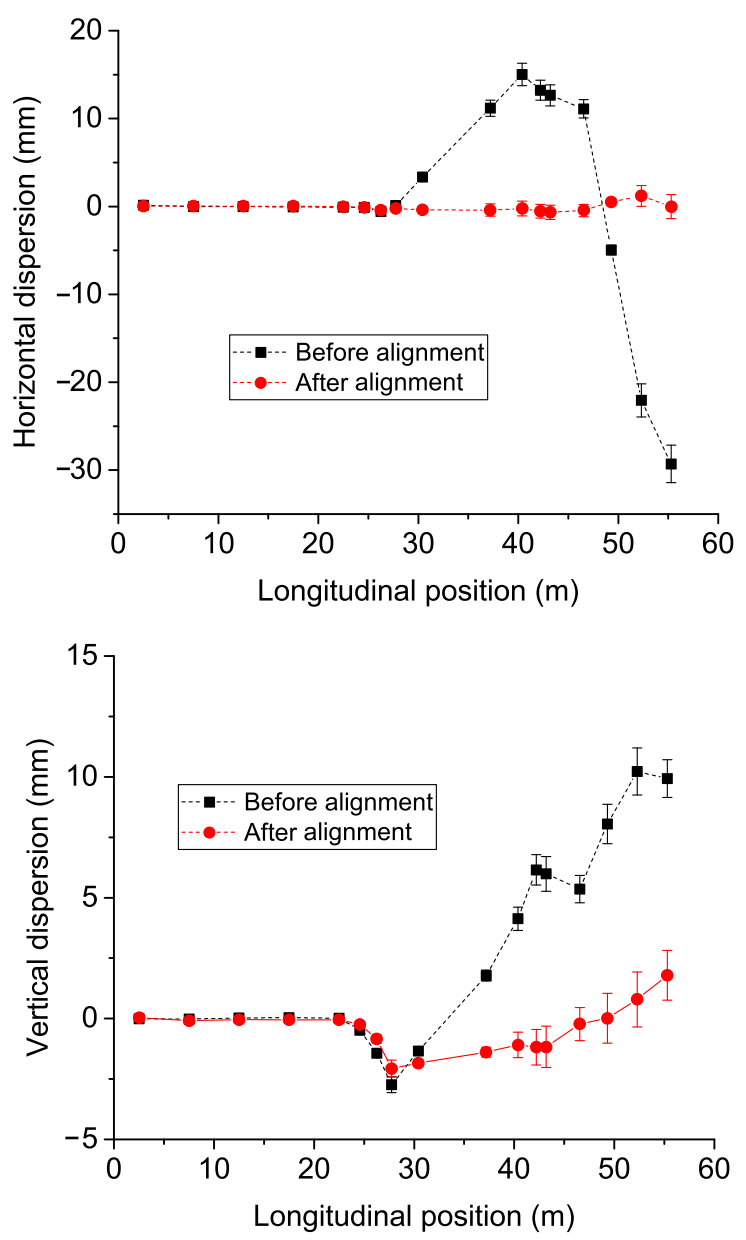

FIG. 55. Measured spurious dispersion in the horizontal (upper panel) and vertical plane (lower panel) along the injector before and after the beam-based alignment (adjustment of the reference orbit).

\section{Orbit response}

The orbit response matrix relates changes in corrector magnet strengths to the resulting changes in orbit measured at certain positions (the locations of the BPMs). A measurement of the orbit response matrix can be used to calibrate quadrupole and corrector magnet strengths. In contrast to circular accelerators, where a well determined rectangular matrix results in accurate values [147], the calibration in beam transport lines, such as linear accelerators, is compromised by the changing beam energy and by the limited information available for the first elements, i.e., the matrix is only triangular due to the constraints of causality. Nevertheless a measurement of the orbit response matrix still provides useful information.

By comparing the measured orbit response matrix to that obtained from an ideal optics model, we gain an approximate verification of the quadrupole and corrector strengths, thereby confirming the field measurements performed before installation to within a few percent. The intrinsic accuracy of the field measurements is, however, much 
higher, on the order of $0.1 \%$. The orbit response measurement therefore primarily serves as a means to uncover installation errors.

The response matrix is also applied for the correction of beam trajectory errors. Either matrices derived from the optics model or from a response measurement may be used, but the measured response matrix usually results in a better performance, since it takes into account small empirical differences such as BPM calibrations errors.

\section{Bunch compressor characterization}

The magnetic chicane is characterized by both transverse and longitudinal dispersions $\left(R_{56}\right)$. The transverse dispersion is monitored by the two BPMs installed in either arm of the chicane (see next section). The longitudinal dispersion, the most relevant characteristic of the bunch compressor and also called $R_{56}$ for the corresponding element of the transfer matrix, was verified by two independent methods. In both methods, the change $\Delta t$ in arrival time in response to a small change $\Delta p$ in beam momentum is observed after the compression chicane. Depending on the $R_{56}$ of the chicane, bunches with higher energy will arrive earlier than bunches with lower energy, since they pass the chicane on a shorter path, according to

$$
c \Delta t=R_{56} \frac{\Delta p}{p},
$$

where $c$ is the speed of light. The first, destructive method uses the temporal modulation of the transverse beam position by the transverse deflecting cavity, measured by a BPM downstream of the cavity, to derive $\Delta t$. In the second, nondestructive method, the change in arrival time is directly recorded by the BAM described in Sec. V G.

Figure 56 shows the results of the two measurements, for various bunch compressor angles between $0^{\circ}$ and $5^{\circ}$, compared to model expectations. The measurement was performed at a bunch charge of $45 \mathrm{pC}$ and a beam energy of about $200 \mathrm{MeV}$, varied within a range of $\pm 1.5 \%$ by adjusting the rf power in the last two S-band structures. All booster structures were operated on-crest for this

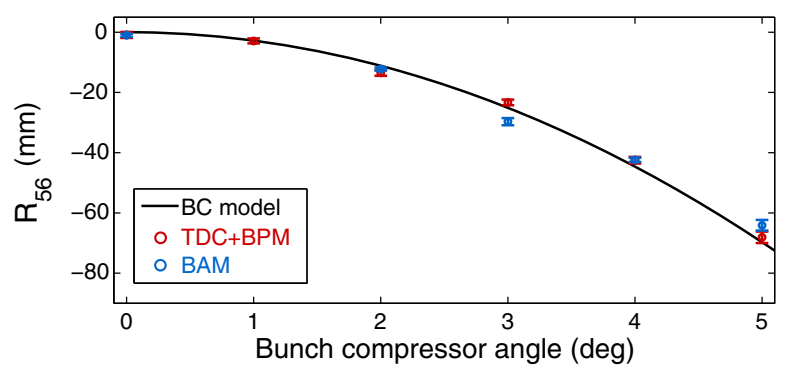

FIG. 56. Comparison of measurements of the $R_{56}$ parameter using the transverse deflecting cavity (TDC) and a downstream BPM (red points) and via bunch arrival-time using a BAM (blue points), for different bunch compressor angles. measurement, i.e., the bunches were not compressed in the chicane.

\section{Geomagnetic field compensation}

The bunch compressor main dipoles are equipped with individually powered correction coils, designed to compensate possible differences in field integral between the four magnets (see Sec. IV E). Since the main dipoles did not require much correction, we utilize the corrector dipoles also for the compensation of the geomagnetic field, which is not negligible at our beam energy. The SITF is almost north-south oriented, such that the effect is mostly in the horizontal plane.

Measuring the geomagnetic field with beam we find a vertical field component of about $30 \mu \mathrm{T}$. This results in a trajectory offset of about $0.5 \mathrm{~mm}$ at the BPMs situated in the bunch compressor arms. By setting the correction coil currents to values such that the additional integrated field corresponds to the integrated vertical geomagnetic field we reduce the trajectory offset to almost zero. To first order, the compensation is valid for any beam energy, since we directly compensate one field by another. The integrated geomagnetic field along the bunch compressor is about $3 \times 10^{-4} \mathrm{Tm}$ whereas the field differences between the magnets amount to about $1.5 \times 10^{-4} \mathrm{Tm}$ [35]. The dispersion chicane is almost perfectly closed once the correction is applied as shown in Fig. 57.

\section{B. Transverse beam characterization}

One of the prime objectives of the SITF consisted in the demonstration of the beam parameters needed for driving the SwissFEL linac and undulator lines to reach the desired radiation power at short wavelengths. A key parameter in this context is the transverse emittance of the electron beam, as it has a strong influence on the FEL power that can

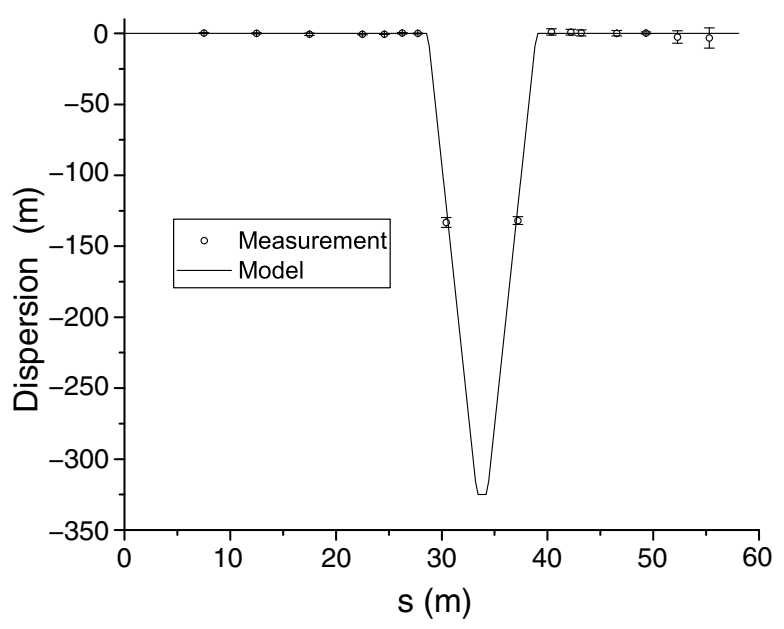

FIG. 57. Measured and modeled dispersion along the injector, with the bunch compressor angle set to $4.0^{\circ}$. 
be reached with a given undulator length [148]. Consequently, a substantial fraction of the operational life of the SITF was devoted to the characterization and optimization of the electron beam's transverse emittance. In particular the emittance of individual longitudinal bunch slices in the core of the bunch, the so-called slice emittance, should be kept as small as possible to enable an optimal lasing process in the undulator section. The projected emittance, i.e., the emittance of the whole bunch, should also be reasonably small to prevent the deterioration of the FEL performance due to transverse offsets between slices and optics mismatch along the bunch. Moreover, if the projected emittance is of the same order as the slice emittance, the optics mismatch between the core and projected beams is reduced. Therefore, the procedure for the overall optics matching is simplified, as in this case it is sufficient to consider the projected parameters.

We measure both projected and slice emittance by means of beam-optical methods [149], whereby the moments of the phase-space distributions are determined from beamsize measurements under varying phase advance between a reference and an observation point, the latter given by the measurement screen. The measured rms beam sizes $\sigma_{x, i}$ $(i=1, \ldots, N)$ depend both on the respective beam transport matrices $R^{i}$ as well as on the initial beam moments $\left\langle x_{0}^{2}\right\rangle$, $\left\langle x_{0}^{\prime 2}\right\rangle$, and $\left\langle x_{0} x_{0}^{\prime}\right\rangle$ at the reference point according to

$$
\sigma_{x, i}^{2}=R_{11}^{i}{ }^{2}\left\langle x_{0}^{2}\right\rangle+R_{12}^{i}{ }^{2}\left\langle x_{0}^{\prime 2}\right\rangle+2 R_{11}^{i} R_{12}^{i}\left\langle x_{0} x_{0}^{\prime}\right\rangle,
$$

where $x$ and $x^{\prime}=d x / d s$, referring to transverse coordinate and trajectory slope, respectively, are defined for the horizontal plane, but an equivalent formalism is valid for the vertical plane $\left(y\right.$ and $\left.y^{\prime}\right)$. The subscript 0 denotes the reference point. It follows that a sufficiently large set of beam size measurements $\sigma_{i}$ obtained under known transport conditions $R^{i}$ allows the reconstruction of the beam moments. While in principle three such measurements would be sufficient to reconstruct the beam moments, in practice we perform many more measurements and let a numerical least-square fit find the beam moments that best describe the observations, to improve the accuracy of the reconstruction. From the beam moments, it is straightforward to derive the geometric rms emittance $\varepsilon_{x}$ as

$$
\varepsilon_{x}=\sqrt{\left\langle x_{0}^{2}\right\rangle\left\langle x_{0}^{\prime 2}\right\rangle-\left\langle x_{0} x_{0}^{\prime}\right\rangle^{2}}
$$

which may be normalized with respect to the beam energy according to

$$
\varepsilon_{n, x}=\frac{p}{m_{e} c} \varepsilon_{x}
$$

with $p$ the beam momentum, $m_{e}$ the electron mass and $c$ the speed of light. Similarly, the Twiss parameters at the reference point are obtained as $\alpha_{x, 0}=-\left\langle x_{0} x_{0}^{\prime}\right\rangle / \varepsilon_{x}$, $\beta_{x, 0}=\left\langle x_{0}^{2}\right\rangle / \varepsilon_{x}$, and $\gamma_{x, 0}=\left\langle x_{0}^{\prime 2}\right\rangle / \varepsilon_{x}$.

We use two methods to vary the phase advance between the reference and the observation point. In the first method, mainly used for the routine measurement of projected emittance, a single quadrupole is used in the same scan to generate phase advance simultaneously and equivalently in the horizontal $(x)$ and the vertical $(y)$ plane. This socalled symmetric single-quadrupole scan [150] requires a particular symmetric beam optics (identical in both planes) at the scanning quadrupole with $\beta$ equal to $\alpha$ times the distance $L$ between the quadrupole and the observing screen, i.e., $\beta_{x}=\beta_{y}=\beta_{0}=\alpha_{0} L$ with $\alpha_{0}=\alpha_{x}=\alpha_{y}$. Under these conditions the beam waist occurs in both planes at the location of the screen for zero gradient field in the quadrupole. In the thin-lens approximation the minimum $\beta$ is given by $\beta_{\min }=L^{2} / \beta_{0}$. In our setup we choose $L=10.83 \mathrm{~m}$ and $\beta_{0}=15 \mathrm{~m}$, therefore $\alpha_{0}=1.385$ and $\beta_{\min }=7.82 \mathrm{~m}$. Thanks to its symmetry this novel method has proven to be fast and intuitive and thus has quickly become the standard method for routine emittance measurements at the SITF.

If greater freedom in the control over the measurement optics is required, as is the case in time-resolved measurements with a streaked beam (Sec. VIC), or the determination of the full set of transverse beam parameters including correlations between the two planes (4D beam matrix) is desired [151], then the use of several quadrupoles [152] to control the phase advance independently in two planes, while fulfilling additional constraints, e.g., on the $\beta$-functions, is inevitable. For this more elaborate method we use three to five quadrupoles downstream of the compression chicane to set up the different beam transport schemes required for the beam optics measurements set out in Eq. (1).

In both methods, the beam originating from the booster should be matched to the optics required for the measurement to minimize the reconstruction errors. This is done by setting the currents of a set of matching quadrupoles upstream of the bunch compressor to appropriate values, normally obtained from a matching tool based on a suitable online machine model. The quality of the optics matching is evaluated from a comparison between the measured Twiss parameters $\alpha, \beta$, and $\gamma$ and the corresponding design values $\alpha_{D}, \beta_{D}$, and $\gamma_{D}$, usually quantified by the mismatch parameter [149], defined as $\xi=\left(\beta_{D} \gamma-2 \alpha_{D} \alpha+\gamma_{D} \beta\right) / 2$ and approaching 1 for a well matched beam. Optics measurement and matching are iterated until a satisfactory result is obtained. As a rule, we only consider emittance measurements to be valid if the mismatch parameter is below 1.1 .

For most of our emittance measurements we have used the novel profile monitor described in Sec. V D 3 to obtain the transverse beam sizes necessary for the evaluation based on Eq. (1). We thereby approximate the rms width of 
a given beam profile in one dimension by the rms width (standard deviation) of a Gaussian function fitted to the corresponding projection of the background-subtracted beam image. This fast and robust approach has proven to be fully adequate in the context of the optimization of an FEL driver, where small contributions from far outlying tails in the beam profiles are not relevant. More elaborate rms methods applied to appropriately noise-filtered beam images (see Refs. [153-155]) were found to give no significant advantages.

While the accurate absolute measurement of beam sizes is essential for the derivation of the beam emittance, it is not imperative for the determination of the beam optics (Twiss) parameters. Indeed, since the Twiss parameters $\alpha, \beta$, and $\gamma$ do not explicitly depend on the absolute beam size but only on its variation along the beam line, it is possible to derive them from relative beam size measurements alone, in particular from the identification of beam waists. A corresponding method was developed and successfully tested at the SITF [156]. It proved useful during the earliest beam commissioning stages when the profile monitors were still subject to calibration.

\section{Longitudinal beam characterization and time-resolved measurements}

The experimental verification of short bunch lengths as well as the measurement of key beam parameters (optics, emittance, energy and energy spread), as a function of the longitudinal position along the bunch, are of fundamental importance for the development of accelerators applied as FEL drivers. At the SITF our main method for bunch length and time-resolved measurements was based on the well established rf streaking technique [66,67] using a transverse rf deflector (see Sec. IV C 4) operating at the same rf frequency as the booster linac and at the zero-crossing phase of the field to achieve a strong correlation between the longitudinal (time) and vertical coordinates along the bunch. This correlation then allows for the measurement of the current profile along the bunch as well as the horizontal emittance of individual slices using regular transverse profile monitors (see Secs. IV F 3 and V D).

Similarly to the transverse beam size, we typically determine the rms bunch length from a Gauss fit to the background subtracted vertical profile of the streaked beam, although other methods for estimating the width of the distribution, e.g., based on the full-width at halfmaximum or rms values, may also be applied. The relation between vertical distance on the screen and time is derived from a comparison of different beam images, obtained at slightly different rf phases of the S-band deflecting cavity, for which $1^{\circ}$ phase change amounts to a time difference of $927 \mathrm{fs}$.

The unstreaked beam can feature a correlation between the vertical and time coordinates, which can be caused, for instance, by wakefields in the accelerating structures. The reconstructed bunch length would be biased for an initially tilted bunch if the measurement is only performed at one zero crossing of the deflecting rf field. We overcome this problem by performing the measurement at both zero crossings and comparing the two measurements following the procedure described in Ref. [67]. In addition to the unbiased bunch length, this method also provides an estimate of the incoming beam tilt. Figure 58 shows an example of a bunch length measurement for an uncompressed beam.

The combination of vertical streaking and horizontal dispersion, in our case in the high-energy spectrometer, allows for a complete mapping of the longitudinal phase space (time and energy). The visualization of the longitudinal phase space in real time constitutes a valuable diagnostic when setting up the compression of the beam (see Sec. VII C).

For the measurement of the optical parameters and emittance of individual slices along the bunch a rectangular region of interest is drawn around the streaked beam and
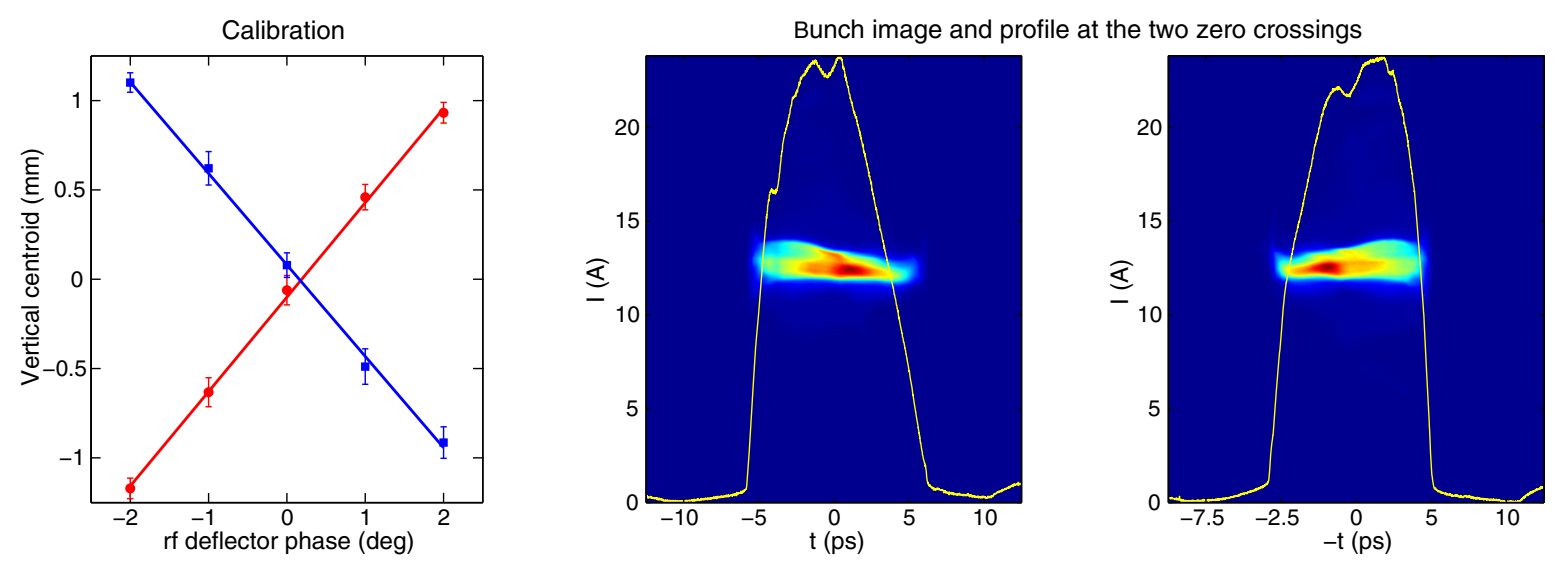

FIG. 58. Example of a bunch length measurement with uncompressed beam, showing the calibration measurement (left plot) and the current profiles overlaid on the images of the streaked beam, as observed at the two zero crossings of the rf deflector (right plots). 
subdivided into horizontal bands corresponding to the bunch slices. Typically 10 slices per rms bunch length are chosen, but this number may vary depending on the beam characteristics or measurement purpose. During optics scans, it is important to associate measurements of the same physical slice along the bunch with one another, regardless of fluctuations in beam arrival time or deflector rf phase. This requirement is satisfied by a slicing algorithm relying on beam-based reference points, see Ref. [148] for details.

Once the beam is resolved into individual slices, the same optics scanning procedure for the determination of the optical parameters and the beam emittance described in the previous section may be applied. In this case, the quadrupole settings used to provide the necessary phase advance for the emittance measurement, while controlling the beam size on the observation screen, are further adjusted to keep the longitudinal (slice) resolution approximately constant during the scan. As in the case for time projected measurements, we again aim for mismatch parametersnow determined individually for each slice-smaller than 1.1, at least in the relevant central part of the bunch.

Instead of using an $\mathrm{rf}$ deflector, the correlation between time and transverse position may also be obtained by a combination of transverse dispersion and energy chirping $[157,158]$. This alternative method was further developed and generalized at the SITF [159]. It has the distinctive advantages of enabling the measurement of slice parameters in both the vertical and the horizontal planes and anywhere along the machine where dispersion can be generated. The method has the limitations that it requires absolute calibration and an energy chirp along the bunch, which precludes its use in conjunction with a spectrometer to map the bunch's longitudinal phase space in general.

\section{BEAM PHYSICS RESULTS}

As one of the principal objectives of the SITF consisted in the demonstration of a high-brightness beam capable of driving a SASE FEL, the beam development program focused on minimizing the transverse beam emittance at the source and on preserving the small emittance under beam transport, acceleration, and, in particular, compression.

The optimum matching of electron and photon beam resulting in transversely coherent FEL radiation in the undulator line imposes the condition $\varepsilon_{n} \lesssim \gamma \lambda / 4 \pi$ [160] on the normalized transverse emittance, $\varepsilon_{n}$, where $\gamma$ is the electron beam's Lorentz factor and $\lambda$ the FEL radiation wavelength. Therefore the final beam energy required to drive an FEL at a given radiation wavelength is dictated by the transverse emittance that can be reached in the rf photoinjector and preserved during beam transport and compression. Beyond the beam energy requirement, a smaller emittance also results in higher radiation power and allows FEL saturation to be reached with a shorter undulator beam line [148].
In this section we describe our various efforts to optimize the beam emittance originating from the source (Sec. VII A), as well as further down the accelerator beam line, both for uncompressed (Sec. VII B) and compressed beams (Sec. VII C), and summarize the beam parameters we were able to achieve with the SITF.

\section{A. Source characterization}

The electron emission process at the gun cathode sets a fundamental lower limit on the achievable beam emittance and thus has a strong influence on the final emittance of $\mathrm{rf}$ photoinjectors. For instance, simulations performed for SwissFEL indicate that for an optimized machine, around $70 \%$ of the final emittance is due to the emittance related to the cathode, also called intrinsic emittance. At the SITF much effort went into characterizing the intrinsic emittance of cathodes and finding ways to minimize it.

The intrinsic emittance of the cathode is related to the initial kinetic energy or effective temperature of the electrons emitted from the cathode. For laser-illuminated photocathodes, made of metal or semiconductor, the intrinsic emittance may be expressed as [161-163]

$$
\varepsilon_{\text {int }}=\sigma_{l} \sqrt{\frac{2 E_{K}}{3 m_{e} c^{2}}},
$$

where $\sigma_{l}$ is the rms laser beam size, $E_{K}$ the average kinetic energy of the photo-emitted electrons, and $m_{e} c^{2}$ the electron's rest mass energy. The ratio $\varepsilon_{\text {int }} / \sigma_{l}$ is independent of the laser beam size and may be called the specific intrinsic emittance. The electrons' average kinetic energy $E_{K}$ depends on the laser photon energy $\phi_{l}$, material properties and the applied electric field. It takes on a different form for metals and semiconductors. For metal cathodes we have

$$
2 E_{K}=\phi_{l}-\phi_{w}+\phi_{\mathrm{Sch}},
$$

with the work function $\phi_{w}$, whereas for semiconductors we have

$$
2 E_{K}=\phi_{l}-E_{g}-E_{a}+\phi_{\mathrm{Sch}},
$$

where $E_{g}$ and $E_{a}$ refer to gap energy and electron affinity, respectively. In both cases the kinetic energy is enhanced by the reduction of the potential barrier due to the applied electric field, the so-called Schottky effect. This contribution, denoted as $\phi_{\text {Sch }}$, is given by [164]

$$
\phi_{\mathrm{Sch}}=\sqrt{\frac{e^{3}}{4 \pi \varepsilon_{0}} \beta E_{c}},
$$

where $e$ is the electron charge, $\varepsilon_{0}$ the vacuum permittivity, $\beta$ the local field enhancement factor accounting for cathode 
surface effects, and $E_{c}$ the rf field on the cathode applied during the extraction of the electrons.

Apart from the cathode surface quality (described by $\beta$ ), the intrinsic emittance of a photoinjector is thus influenced by three main parameters under our control: (i) the photon energy, given by the wavelength of the gun laser, affecting directly the energy of the photo-emitted electrons, (ii) the applied field on the cathode, determining the size of the Schottky effect, and (iii) the cathode material, specifying the work function in the case of a metal or the gap energy and the electron affinity in the case of a semiconductor. At the SITF we have performed experimental studies on all three relationships: we confirmed, by using the wavelengthtunable Ti:sapphire laser system described in Sec. IVA, that the intrinsic emittance decreases with laser wavelength [165]; we observed the reduction of intrinsic emittance as a function of the applied rf gradient [166]; and we compared the intrinsic emittances and general operational performance of metal (copper) and semiconductor (cesiumtelluride) cathodes [44].

In all these studies we extract the specific intrinsic emittance $\varepsilon_{\text {int }} / \sigma_{l}$ from measurements of the emittance at the final booster energy as a function of the laser beam size on the cathode, controlled by adjusting the size of a circular laser aperture. Special care must be taken to minimize or subtract possible contributions to the emittance arising after the emission at the cathode, e.g., from space-charge and rffield effects.

To eliminate space-charge effects, the measurements are performed at very low bunch charges, well below the spacecharge limit as confirmed experimentally. During a laserbeam-size scan, the bunch charge is adjusted to keep the surface charge density constant. Possible chromatic and dispersive effects along the bunch are minimized by keeping the electron energy approximately constant along the bunch by means of on-crest acceleration in the booster. Furthermore, to avoid a possible impact on the projected emittance from the gun rf field variation experienced by the bunch along its longitudinal extension, we only consider the slice emittance at the core of the bunch. This core slice emittance is obtained by dividing the bunch into typically ten slices per rms bunch length and averaging the measured slice emittance over an appropriate number of slices around the longitudinal center of the bunch.

Most of our intrinsic emittance measurements were performed with the CTF gun (see Sec. IV C 1), which features a symmetric rf feed design to avoid dipolar kicks, but has no compensation of quadrupolar fields acting on the electron beam. These quadrupolar fields lead to a quadratic rise of the emittance as a function of the laser spot size. We take this additional component into account by fitting a quadratic function to the measurements, thereby obtaining the specific intrinsic emittance as the linear coefficient of the fit function. Additional measurements performed with the new SwissFEL gun show that the quadratic effect is less

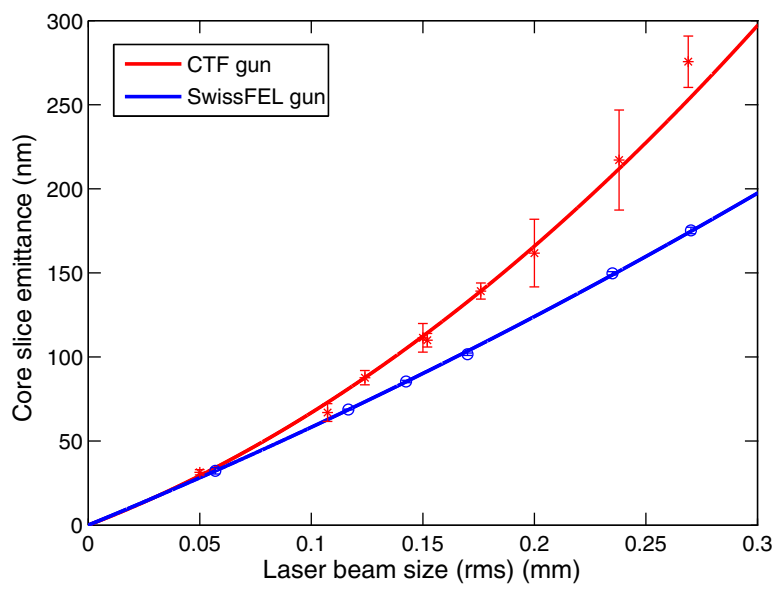

FIG. 59. Two examples of aperture scans showing core slice emittance measured for various laser spot sizes with the CTF gun (red asterisks) and the SwissFEL gun (blue circles). The dependence observed for the CTF gun features a distinct quadratic component (see text for details). In both cases the electrons were extracted from a copper cathode.

pronounced in this case. In Fig. 59 we compare two examples of aperture scans performed with the two rf guns.

The most relevant results of our systematic emittance studies with copper cathodes are that the intrinsic emittance of such cathodes can indeed be reduced by about $20 \%$ when tuning the laser wavelength from $262 \mathrm{~nm}$ to $276 \mathrm{~nm}$ [165] and that a similar reduction can be achieved by lowering the $\mathrm{rf}$ gradient at the extraction phase from $49.9 \mathrm{MV} / \mathrm{m}$ to $16.4 \mathrm{MV} / \mathrm{m}$ [166]. The wavelength tuning measurements confirm earlier studies [167] carried out with a combined diode rf electron gun [168].

These observed reductions apply to individual cathodes measured under the same operating conditions within a relatively short time. Measuring various cathodes at different times with respect to their installations we found that variations in intrinsic emittance between different cathodes, originating, e.g., from differences in surface quality, as well as degradation effects are also in the $20 \%$ range. Therefore such effects can easily outweigh any benefits gained from laser-wavelength or rf-gradient tunings, which, however, come at the expense of significant losses in quantum efficiency.

Similarly, our comparison between copper and cesium telluride cathodes resulted in emittance values for the two types of cathodes that lie in the same range [44]: for both cathode materials we obtained values between about $0.4 \mu \mathrm{m} / \mathrm{mm}$ and $0.6 \mu \mathrm{m} / \mathrm{mm}$ for the specific intrinsic emittance. Earlier measurements performed on copper cathodes in the context of the development of the LCLS at SLAC generally gave higher values [169-172], whereas a previous PSI experiment based on a combined diode $\mathrm{rf}$ electron gun resulted in a specific intrinsic emittance of around $0.5 \mu \mathrm{m} / \mathrm{mm}$ for this material $[167,168]$. In the case of cesium telluride, experiments at the photoinjector test 
facility at DESY, Zeuthen (PITZ), also indicated a higher specific intrinsic emittance [6], whereas estimates based on the observed electron emission spectrum [173] agree with our measurements.

Some of the laser-beam-size scans involved emittance measurements at extremely low bunch charges. For instance, the measurement with the smallest laser aperture at the lowest rf gradient was done on a bunch with only $30 \mathrm{fC}$ charge. The beam slices, for which individual emittances were determined in this measurement, contain around $1 \mathrm{fC}$ of charge; in other words they are made up of a few thousand electrons only. The measured normalized core slice emittance in this case was slightly below $25 \mathrm{~nm}$ [166].

Given our experimental results on intrinsic emittance, the options of tuning the wavelength of the gun laser or the rf gradient of the photoinjector gun will not be pursued further at SwissFEL. Moreover the baseline cathode material for the SwissFEL injector has been changed from copper to cesium telluride, since it provides a quantum efficiency about two orders of magnitude higher at comparable intrinsic emittance (see also Sec. IV B). The copper option will be maintained nevertheless, for the case of major problems with the production or operation of the cesium telluride cathodes.

\section{B. Optimization of uncompressed beam}

Apart from the mitigation of emittance dilution effects, the optimization of an rf photoinjector involves trade-offs between the contributions to the beam emittance related to the intrinsic emittance at the cathode, space charge and rf fields. To minimize the emittance at the SITF, we first performed a numerical optimization followed by a full empirical optimization of the actual machine.

The numerical optimization is based on simulations using the ASTRA code [127] coupled to a dedicated optimizer developed for SwissFEL [174]. The optimizer minimizes both the emittance and the mismatch along the central part of the bunch (75\% of the total length) at the exit of the second booster structure, via the construction of an appropriate figure of merit. The main parameters to be optimized are the distance between the gun and the first booster structure, the transverse laser spot size and the gun solenoid focusing strength. In the optimization procedure, as applied to the SITF design, the gun gradient is kept at its nominal value (see Sec. IV C 1) and also the bunch length at the cathode (determined by the laser pulse length) is fixed to the original design values (FWHM) of $9.9 \mathrm{ps}$ for the $200 \mathrm{pC}$ bunch and $3.7 \mathrm{ps}$ for the $10 \mathrm{pC}$ bunch [3,20]. One of the most important consequences of the numerical optimization was an increase of the distance between the gun and the first booster structure by about $20 \mathrm{~cm}$ [174] with respect to the original design, which was based on the Ferrario working point [23].
The laser spot size determined by the numerical optimization for the $200 \mathrm{pC}$ case serves as a starting point for the empirical optimization. The $10 \mathrm{pC}$ bunch is simply reduced in all dimensions to have the same threedimensional charge density. Based on the simulation results we select the laser aperture that gives a laser beam size as close as possible to the best value. Later we verify empirically the optimality of the aperture size. The longitudinal laser profile is an approximated flat-top profile obtained by pulse stacking of 32 replicas (Fig. 4 shows the profile for 16 replicas). Further manual optimization steps, after the careful beam-based alignment of the laser beam on the cathode and the gun solenoid to the gun rf axis, as described in Sec. VI A 1, include: (i) the fine adjustment of the transverse laser profile towards maximum azimuthal symmetry and homogeneity (see Sec. IVA); (ii) the setup of the longitudinal laser profile via pulse stacking (see again Sec. IVA) to approximate a flat-top distribution, which ensures that most of the longitudinal beam slices have the same emittance and optics, thus resulting in a small projected emittance (in contrast to, e.g., a Gaussian distribution); (iii) the setting of the gun rf phase and gradient to obtain the smallest beam energy spread around the $7.1 \mathrm{MeV}$ design energy as observed with the gun spectrometer; (iv) the empirical optimization of the gun solenoid excitation current to achieve minimum emittance through invariant envelope matching; (v) the systematic correction of cross-plane coupling terms with two corrector quadrupole magnets (normal and skew) integrated into the gun solenoid and four solenoids around the first two S-band accelerating structures [151]; (vi) the centering of the orbit in the S-band booster structures, as described in Sec. VIA 1 , to minimize wakefield contributions to the emittance; (vii) the empirical correction of spurious dispersion downstream of the booster affecting the emittance measurement via the generation of local orbit bumps.

After applying this optimization procedure we measured, for the $200 \mathrm{pC}$ bunch charge case, a normalized projected emittance of about $300 \mathrm{~nm}$ and a normalized slice emittance of about $200 \mathrm{~nm}$. For a bunch charge of $10 \mathrm{pC}$, the optimized values were $140 \mathrm{~nm}$ and $100 \mathrm{~nm}$ for projected and slice emittance, respectively. These results, first obtained with the CTF gun, have been confirmed with the SwissFEL gun, and in some cases even improved upon. For instance, during the last operation run in 2014 a slice emittance of around $100 \mathrm{~nm}$ was also achieved for a bunch charge of $20 \mathrm{pC}$. This measurement is presented in Fig. 60; measurements at 10 and $200 \mathrm{pC}$ can be found in Ref. [148]. The obtained experimental results for both slice and projected emittance proved to be stable under repeated measurements, as shown in Fig. 61, and reproducible from scratch as long as the laser quality remains the same. For a more detailed account of our emittance optimization procedure, as well as a discussion of measurement resolutions and errors, we refer to Ref. [148]. 


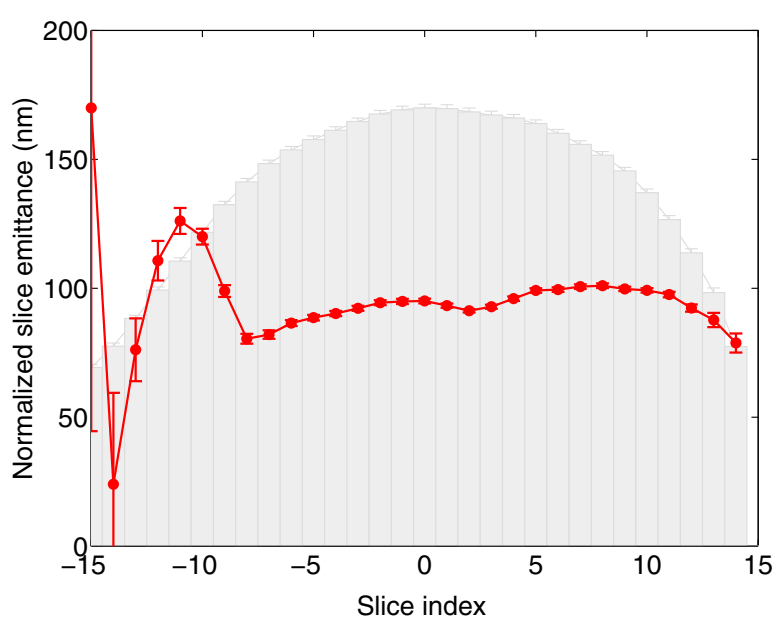

FIG. 60. Normalized slice emittance measured with the SwissFEL gun for a bunch charge of $20 \mathrm{pC}$ (red). The longitudinal charge profile is shown as gray bars.

In the optimization procedure described above, the laser pulse length defining the bunch length is treated as a given, originating from the design of the machine $[3,20]$. In the last operating months of the SITF, however, we performed a systematic study of the effect of the laser pulse length on the emittance both for uncompressed and compressed beams. The study was carried through with the SwissFEL gun using a cesium telluride cathode. Optimizing the beam separately for the design case with a laser pulse length of 9.9 ps and for two additional cases with laser pulse lengths of $5 \mathrm{ps}$ and $2.5 \mathrm{ps}$ (FWHM), we found no improvement either in emittance or in brightness when going to shorter laser pulse lengths.

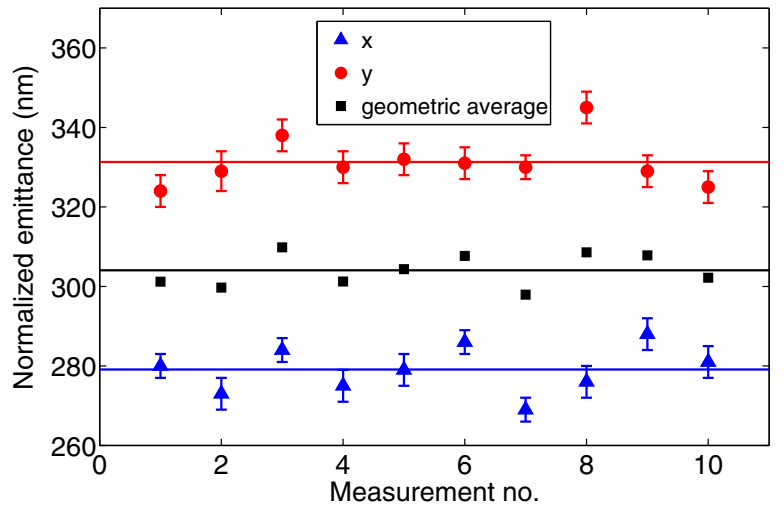

FIG. 61. Normalized horizontal (blue) and vertical (red) projected emittance obtained for a bunch charge of $200 \mathrm{pC}$ from ten consecutive measurements with the CTF gun, illustrating the stability of machine and diagnostics chain. The geometric mean of the horizontal and vertical results is shown in black. The continuous horizontal lines represent the averages over the ten measurements.

\section{Bunch compression and emittance preservation}

Bunch compression utilizing path length differences in a dispersive section, such as a magnetic chicane, has become a well-established technique (see, e.g., Refs. [175,176]). The SwissFEL design foresees two such bunch compressors to achieve final rms bunch lengths between 2 and $25 \mathrm{fs}$, depending on the bunch charge. The first stage of this compression scheme was tested at the SITF with the magnetic chicane described in Sec. IV E. Indeed the demonstration of bunch compression with acceptable degradation of beam quality represented one of the main goals of the SITF experimental program.

\section{Compression procedure}

Our compression setup procedure is based on an analytical derivation of the rf parameters needed to arrive at a certain bunch length for a given configuration $\left(R_{56}\right)$ of the bunch compressor. In this procedure the first two booster structures are used for acceleration only (operating at their on-crest phases), whereas the last two booster structures are used for acceleration and energy chirping (operating at an appropriate common off-crest phase). The higher-harmonic X-band structure is operated at the oncrest phase providing maximum deceleration to linearize the longitudinal phase space, including the compensation of second-order terms induced by the bunch compressor. This structure is not normally used to further modify the energy chirp.

For the case of the SITF, with one bunch compression chicane and without strong longitudinal wakefield interactions from C-band linacs, it is possible to derive an analytic description of the compression setup. (For SwissFEL with two bunch compressors and a C-band linac, such a straightforward approach is no longer applicable and a semianalytical model following Ref. [177] will be adopted.)

In practice, we start the compression setup by determining the incident energy before the chirping structures, which is necessary in order to compute the appropriate compression parameters for a given target bunch length. Our derivation of the compression parameters also assumes zero incoming energy chirp. We therefore, in a second step, remove a potential incoming energy chirp originating from the gun by observing the streaked beam in the final spectrometer in response to small variations in the phase of the second booster structure.

To facilitate the ensuing optics matching of the compressed beam, the uncompressed beam at the final energy is matched. We then generate the required energy chirp by going to the predetermined off-crest phase, while simultaneously compensating for the energy loss by increasing the amplitude in the last two structures accordingly. The amplitude of the X-band structure, operating at the on-crest decelerating phase, is then ramped up until its induced energy loss matches the value expected from the 


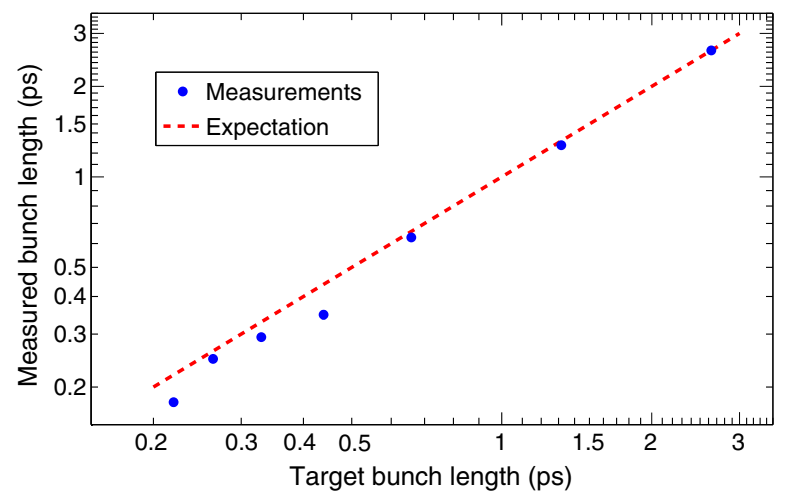

FIG. 62. Measured rms bunch length after applying the compression procedure, compared to the respective target bunch length. (Statistical errors, obtained from repeated measurements, are smaller than the marker circles.)

compression scheme, as confirmed directly with the spectrometer. The associated energy loss is again compensated by a corresponding adjustment in the amplitude of the last two booster structures. Finally, the optics of the compressed beam are rematched, and the compression factor is verified with a bunch-length measurement.

Following this procedure we can reliably establish compression factors up to about 12 with a target accuracy of $10 \%-20 \%$, as shown in Fig. 62. Small adjustments of the compression phase are then sufficient for the further fine tuning of the bunch length.

\section{Phase space fragmentation of the compressed beam}

Preliminary compression experiments, using the flattoplike Ti:sapphire laser pulses built up from pulse stacking (see Fig. 4) to generate electrons from a copper cathode, resulted in a strong fragmentation of the electron phase space into pronounced energy bands. Similar distortions of the longitudinal phase space after compression have been observed at other facilities [178-180]. The phase space fragmentation has an adverse impact on beam emittance and energy spread and compromises the emittance measurement itself. It also aggravates the risk of further beam degradation with respect to FEL performance through the microbunching instability [181]. Figure 63 shows two examples of fragmented phase space distributions, measured in the dispersive section of the high-energy spectrometer while simultaneously streaking the beam with the rf deflector. The measurements presented here were obtained with a laser profile similar to the one shown in Fig. 4.

To avoid complications from this effect without changing the photocathode material, the optimization of the compressed beam, as described in the following section, was continued with the Nd:YLF laser, which delivers relatively smooth Gaussian pulses (see Fig. 3).
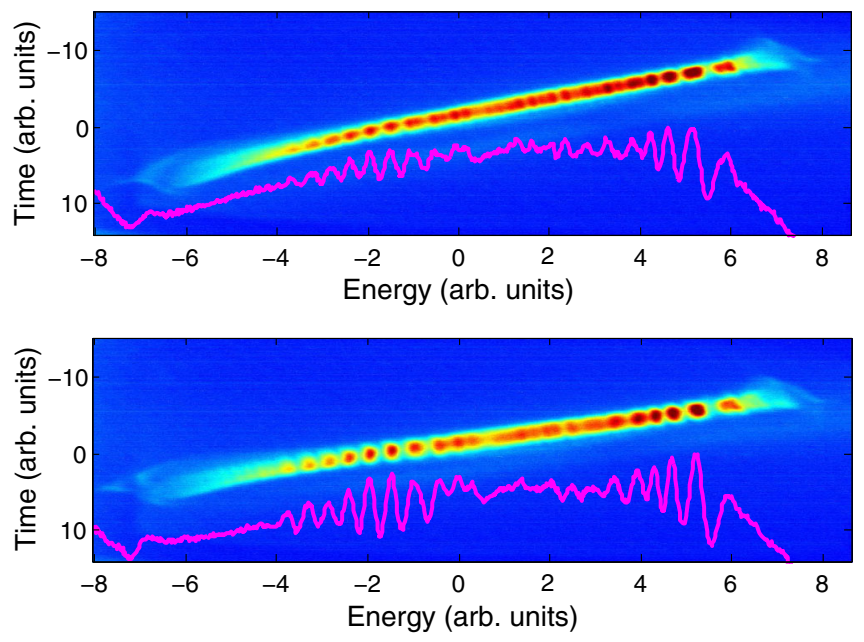

FIG. 63. Examples of phase space fragmentation of compressed electron beams generated from a copper cathode with a flat-top like laser profile obtained with pulse stacking (see Fig. 4). The figures show particle densities of the vertically streaked beam as recorded on a screen in the dispersive section of the high-energy spectrometer for compression factors of 6 (top) and 12 (bottom). The magenta lines are projections of the distributions onto the horizontal (energy) axis, emphasizing the fragmentation effect.

The problem of phase space fragmentation arising from the longitudinal charge modulation induced by the pulsestacked laser profile can also be mitigated by choosing a photocathode material with a response time longer than the characteristic temporal substructure of the laser pulse. At the SITF we have verified that a cesium telluride cathode provides enough time spread to smooth out significantly the phase space of electron bunches generated with a pulsestacked laser profile, even after strong compression, see Fig. 64. This observation provided strong additional support for the choice of cesium telluride as baseline photocathode material for SwissFEL. Further suppression of the microbunching instability at SwissFEL will be achieved with a laser heater system [182] based on conceptual [183] and experimental [184] work carried out for the LCLS project at SLAC.

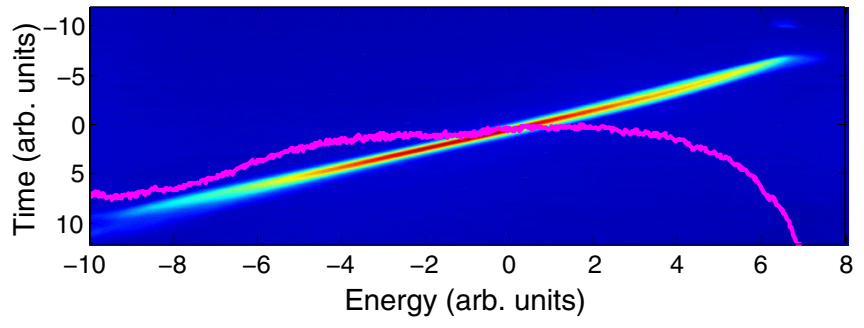

FIG. 64. Example of a compressed beam with smooth phase space distribution as obtained with a cesium telluride cathode using the same pulse-stacked laser profile as in Fig. 63. The applied compression factor is 6 , corresponding to the upper panel of Fig. 63. 

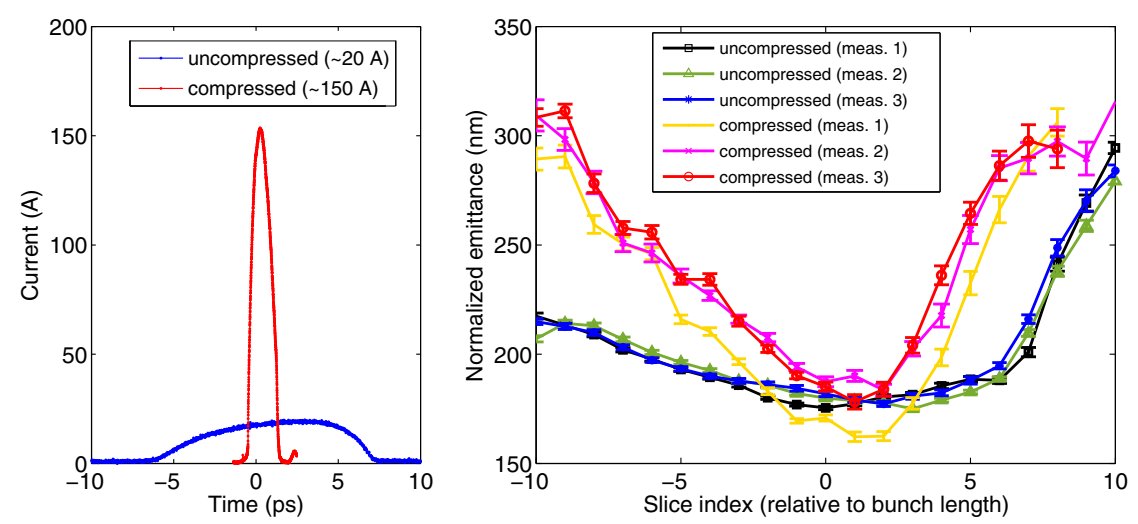

FIG. 65. Current profiles (left plot) and three consecutive slice emittance measurements (right plot) for uncompressed and compressed beams (compression factor 7.5). The longitudinal extension of a slice is a fixed fraction of the rms bunch length.

\section{Optimization of compressed beam}

The first round of emittance measurements on compressed beams, performed with a smooth longitudinal laser profile and at moderate compression factors between 5 and 10 , indicated a significant increase in the core slice emittance resulting from the compression, similar to earlier observations made at FLASH [185] and LCLS [186]. Systematic studies showed that the observed emittance growth is independent of the beam energy, but does increase with the dipole bending angle in the bunch compressor (at constant compression factor) [187]. Deliberately degrading the emittance of the incoming beam we also found that the emittance increase mainly occurs for beams with low initial emittance, whereas an already large emittance is hardly affected by the compression. Furthermore, a strong dependence of the emittance growth on the beam optics along the bunch compressor was observed. For instance, a change of one unit in the horizontal $\alpha$-function at the entrance of the bunch compressor can lead to a relative increase of the core slice emittance of the compressed beam of up to $50 \%$.

Numerical simulations using the CSRTRACK code [188] for the tracking along the bunch compressor qualitatively reproduce the observed behavior, if a 3D model based on the pseudo Green's function [189] is used to describe the electron interactions, but not if the 1D model is selected. From this we infer that the emittance growth is due to 3D effects originating from coherent synchrotron radiation, but possibly also from other sources. A viable mitigation strategy therefore consists in minimizing the horizontal $\beta$-function at the location of the last two dipoles of the chicane, where the bunch length is reduced. By careful tweaking of the beam optics along the bunch compressor, varying the strengths of the last few quadrupoles in the matching section by a few percent or less only, we managed to preserve the core slice emittance to within about $10 \%$ for compression factors up to about 10 . Figure 65 shows the result of three consecutive slice emittance measurements on a compressed beam, together with the current profile.
For comparison, the corresponding measurements before compression are also plotted.

Another conclusion from our studies, reported in detail in Ref. [187], is that great care must be taken when matching the beam in transport sections where it carries a large energy chirp, as is the case in front of a bunch compressor: chromatic effects acting on the energy-chirped bunch can give rise to a large slice mismatch along the bunch, with the effect that only a short part of the bunch fulfills the delicate beam optics conditions required to avoid emittance degradation during compression. A smooth optics in matching sections before bunch compressors, where focusing of an energy-chirped beam is required, is therefore of vital importance.

The final results of our emittance minimization efforts for both compressed and uncompressed beams are summarized in Tables I and II.

\section{Further measurements}

Apart from the beam optimization efforts described above, the SITF was used for a number of other beam dynamics studies as well as for beam-based tests of beam diagnostics and manipulation techniques during its time of operation. The detailed description of these additional developments is beyond the scope of this report and the reader is referred to the corresponding publications for more information.

Further studies performed at the SITF include: (i) the evaluation and implementation of possible collimation strategies after the photocathode gun to limit the dark current transported further downstream [53]; (ii) a proof-ofprinciple experiment to test a new method for measuring longitudinal bunch profiles by utilizing the beam's interaction with the transverse wakefield self-induced by offaxis passage through a dielectric-lined or corrugated waveguide [91] (see also Sec. IV F 4); (iii) the test of a novel method to correct for beam tilts, which exploits dispersive effects in the bunch compression chicane acting on an energy-chirped beam [73]; (iv) a systematic comparison 
between the transverse emittance measurement method based on quadrupole scans and the method using multiscreen data at fixed beam optics in the FODO section [190].

\section{UNDULATOR EXPERIMENT}

The availability of a well-tuned, low-emittance electron beam at the SITF afforded the opportunity to perform a test of the SwissFEL hard-X-ray undulator design under realistic beam conditions. (The design of the SwissFEL soft-X-ray undulators had not yet been finalized at the time of SITF operation.) To this end a prototype of the SwissFEL U15 undulator was installed in December 2013 and tested during the run period lasting from January to April 2014.

\section{A. Motivation and goals}

The undulator test at the SITF provided opportunities (i) to broadly verify - with respect to FEL performanceessential quality features of both the electron beam and the undulator design under real operating conditions [even if only at vacuum ultraviolet (VUV) and optical photon wavelengths], (ii) to validate the undulator magnetic model as well as the results of the magnetic measurements directly with the help of the electron beam, and (iii) to test the undulator alignment concept based on permanent quadrupole magnets.

The primary goals of the experiment were therefore the observation and characterization of undulator radiation arising from the SASE process, the systematic measurement of undulator induced orbit kicks as a function of the magnetic gap to uncover possible flaws in the undulator field, and the demonstration of the undulator alignment procedure.

\section{B. Experiment setup \\ 1. The U15 undulator}

The $4 \mathrm{~m}$ long hybrid in-vacuum U15 undulator features a period length of $15 \mathrm{~mm}$ and a tunable undulator parameter, $K$, between 0.1 and 1.8 , achieved with a movable gap, variable between 3 and $20 \mathrm{~mm}$ by means of a wedge based gap drive system. The U15 was designed for the SwissFEL hard-X-ray beam line based on the experience made at the SLS with hybrid in-vacuum undulators [191,192], with appropriate modifications for the specific requirements of a linac driven FEL [193]. (The general concept of in-vacuum undulators was pioneered by SPring-8 [194].) The relatively large number of identical units (13) planned for SwissFEL called for several design changes in order to benefit from an industrial series production. To provide more rigidity to the magnetic structure, a closed mineral cast frame design was chosen. The mineral cast option was selected in place of the more common metallic frame solution to reduce costs, mechanical vibrations, and disturbances from stray fields. The entire frame is equipped with a five-axis camshaft mover system for remote positioning with micrometer precision.

The new approach introduced additional constraints for the magnetic measurements and necessitated the development of a novel bench for the optimization and characterization of the undulator magnetic properties [195]. The more modest requirements on the field quality for a singlepass machine allows for a smaller good-field region (larger tolerance on multipole components), thereby reducing the magnetic forces and enabling higher $K$ values. A more complete description of the U15 design can be found in Ref. [196].

\section{Undulator preparation}

The U15 prototype undulator was subjected to the same preparation procedure as all U15 undulators built for SwissFEL. The undulator elements are delivered to PSI in three distinct parts: the frame, equipped with the gap drive system, the magnetic array and the vacuum components. For the first set of measurements the magnetic array is assembled inside the frame. The trajectory and the phase errors are tuned adjusting the pole height for the nominal $K$ value $(K=1.2)$. After carefully securing the positions of the magnets within the magnetic array, the unit is removed and equipped with all vacuum components. Once the undulator is assembled in its final configuration, the phase error is remeasured and improved upon if necessary. We then characterize the magnetic array within the entire range of operation, producing models to operate it in the accelerator ( $K$ values and field errors as a function of gap height) [197].

Before removing the undulator from the measurement bench, we position two small permanent quadrupole magnets along the measured undulator magnetic axis, one immediately before and one after the U15 module, to be later used as a reference for the alignment in the accelerator beam line. These alignment quadrupoles are only used during the alignment procedure and are retracted from the beam by a remotely controlled pneumatic system during normal (FEL) operation. Taking the magnetic axis as reference has the advantage of reducing the requirements on the mechanical alignment accuracy.

\section{Installation and instrumentation at the SITF}

To accommodate the U15 prototype in the SITF required substantial modification to the beam line in the diagnostics section. Specifically, one of the $5 \mathrm{~m}$ girders in the FODO channel of the diagnostics section had to be removed and several accelerator components rearranged.

The principal instrumentation needed for the planned undulator measurements consisted of two appropriately positioned BPMs to monitor the effect of the undulator on the electron orbit and three YAG:Ce screens for the observation of the undulator radiation, located downstream of the undulator. The first of these YAG:Ce screens 
included a void area in the lower half to allow the vertically deflected electron beam to pass through without disturbing the photon signal registered in the upper screen area. The scintillating screens did not allow for measurements of the absolute intensity of the photon beam. Additional information was gained from an in-air spectrometer capable of measuring the photon energy in the optical spectrum range.

Throughout the undulator experiment, the radiation dose was constantly monitored by means of dose rate monitors described in Sec. IV I. During the three month period of the experiment an integral dose of about $100 \mathrm{~Gy}$ was recorded. The radiation had no observable effect either on the magnetic or on the electronic components of the undulator system, as was later confirmed with lab measurements following the removal of the undulator from the beam line.

\section{Results}

\section{Undulator alignment}

With the one undulator installed, we define the reference orbit for its alignment simply by the centers of the nearest fixed quadrupoles upstream and downstream of the undulator and calibrate the adjacent BPMs to this orbit. Once the reference orbit has been identified, an alignment procedure is applied to ensure the coincidence of the undulator's magnetic axis with this orbit [198]. For the measurements in the horizontal plane, the undulator gap is set to the nominal $K$ value, where field errors have been minimized by the magnetic optimization. For the measurements in the vertical plane, however, the undulator gap is kept open to avoid the strong focusing effects occurring at the relatively low energies available at the SITF.

Starting from the reference orbit with both alignment quadrupoles retracted, the upstream alignment quadrupole is placed into the beam, and the undulator is moved both horizontally and vertically. Monitoring the downstream BPMs, we then find the position of the undulator for which the reference orbit readings are recovered, i.e., where the upstream alignment quadrupole has no steering effect. After removing the upstream alignment quadrupole, the downstream alignment quadrupole is inserted into the beam and the undulator is adjusted again both horizontally and vertically until the reference orbit is retrieved. Once these two positionings have been determined, the final position and orientation (pitch and yaw angle), for which the undulator is aligned with the reference orbit, can be computed and applied to the mover system. Figure 66 shows an example of an undulator position scan performed as part of the alignment procedure.

\section{Residual field error measurements}

When working with a variable gap configuration the preservation of the undulator's field integrals (governing the integral effect on the electron orbit) for all gap settings is a demanding requirement. It is therefore important to

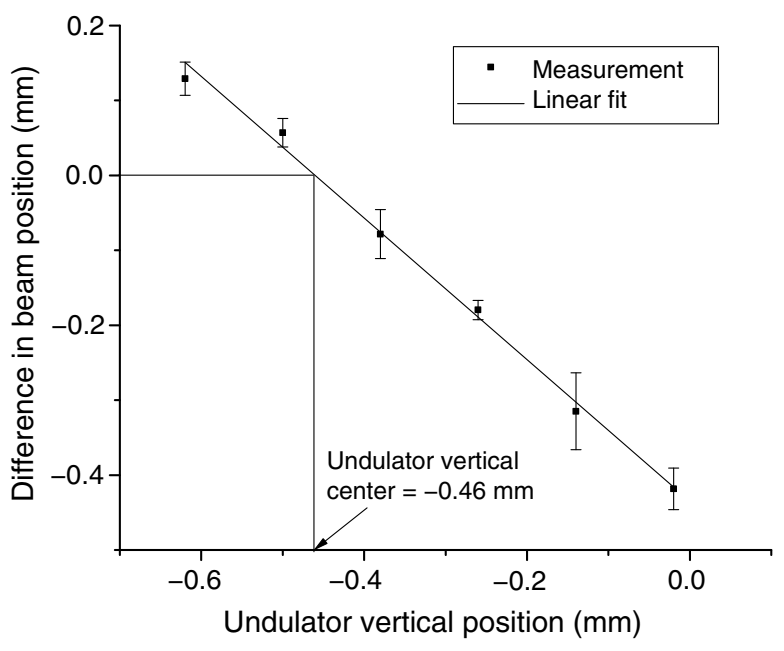

FIG. 66. Example of an undulator alignment scan showing the measured beam positions relative to the reference orbit, $\Delta y$, versus vertical undulator position.

characterize the undulator's net effect on the electron orbit as a function of the gap height to quantify possible residual field errors.

Such residual field errors mainly occur at the entrance and the exit of the undulator, giving rise to corresponding deflections ("kicks") in the electron orbit. If the electrons experience no focusing inside the undulator, these entrance and exit kicks can be inferred from the orbit modification at the undulator exit, both in position and angle $\left(\Delta x\right.$ and $\left.\Delta x^{\prime}\right)$, induced by the setting of the undulator gap to a certain $K$ value. While this change in orbit cannot be measured directly, it can be derived from the orbit response further downstream. In our case we use two dedicated BPMs downstream of the undulator. Moreover, all magnets between the undulator and the two BPMs are switched off. Corrector magnets upstream of the undulator are used to ensure a constant injection orbit. The undulator gap is then reduced from the open position to the position corresponding to a certain $K$ value, and the associated change in the orbit downstream of the undulator is recorded by the BPMs. The measurement is then repeated for different $K$ values.

Figure 67 shows the entrance and exit kicks in the horizontal plane as a function of the $K$ value, derived from downstream orbit measurements at a beam energy of $100 \mathrm{MeV}$. The kicks, which are shown relative to the $K=0$ reference case (not the nominal $K=1.2$ ), exhibit the pattern expected from the central symmetry of the field profile, which is clearly not masked by field errors. At the highest $K$ values (1.8) the kicks amount to about $60 \mu \mathrm{Tm}$, close to predictions based on numerical calculations and magnetic measurements.

The presence of natural focusing in the vertical plane prevents a straightforward interpretation of the orbit response at the undulator exit (in this case $\Delta y$ and $\Delta y^{\prime}$ ) in terms of entrance and exit kicks. Nevertheless a 


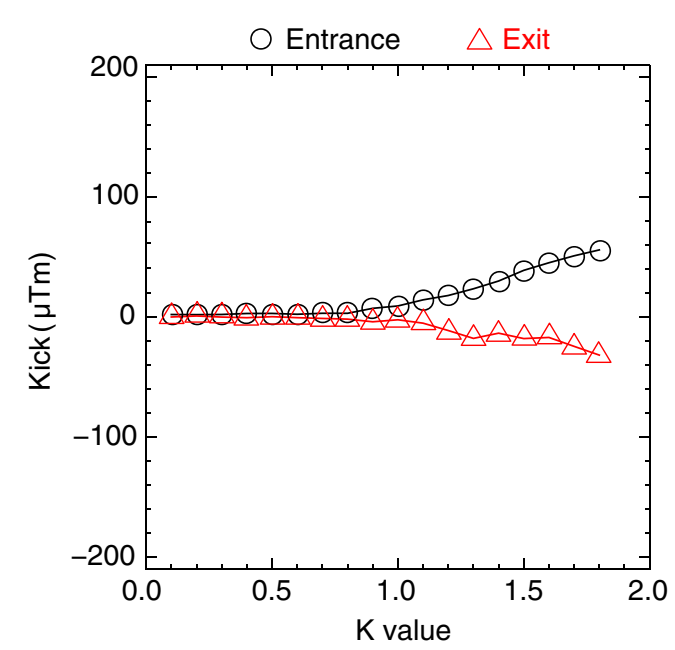

FIG. 67. Undulator entrance (black circles) and exit (red triangles) kicks measured as a function of the undulator $K$ value in the horizontal plane.

comprehensive picture of the combined effects of focusing and possible kicks from residual field errors may be obtained by determining the orbit changes $\Delta y$ and $\Delta y^{\prime}$ not only as a function of the $K$ value, but also, for each $K$ value, as a function of the undulator height and pitch. The resulting set of two-dimensional plots, reproduced in Fig. 68, clearly reveals the quadrupole component, with axis and phase advance both depending on the $K$ value, indicating that the field errors change with the undulator gap.

The overall relatively large orbit deflections in the vertical plane highlighted the need for improvements in the assembly and measurement of the magnetic array. Consequently a new sorting algorithm based on the measurement of individual magnets has been planned and implemented for the next modules with the goal of increasing the quality of the field in the horizontal plane (affecting the vertical electron orbit), thereby reducing the imperfections below specifications. Moreover, the SwissFEL undulators will be equipped with additional vertical steering magnets to counteract any residual vertical orbit kicks.

\section{SASE performance}

Our first attempts to produce FEL radiation from the undulator were made at a beam energy of $220 \mathrm{MeV}$, corresponding to photon wavelengths between $61 \mathrm{~nm}$ (for $K=1$ ) and $106 \mathrm{~nm}$ (for $K=1.8$ ). Soon after setting up the beam transport through the undulator, a bright signal emerged on the first YAG:Ce screen downstream of the undulator, clearly related to FEL radiation. But the asymmetric appearance of the beam spot in the form of a diffraction arc and the fact that it could not be propagated beyond the first screen strongly suggested that only a reflection of the actual FEL signal was observed. Later it became clear that the unexpectedly strong downward kick exerted by the fringe field at the undulator entrance

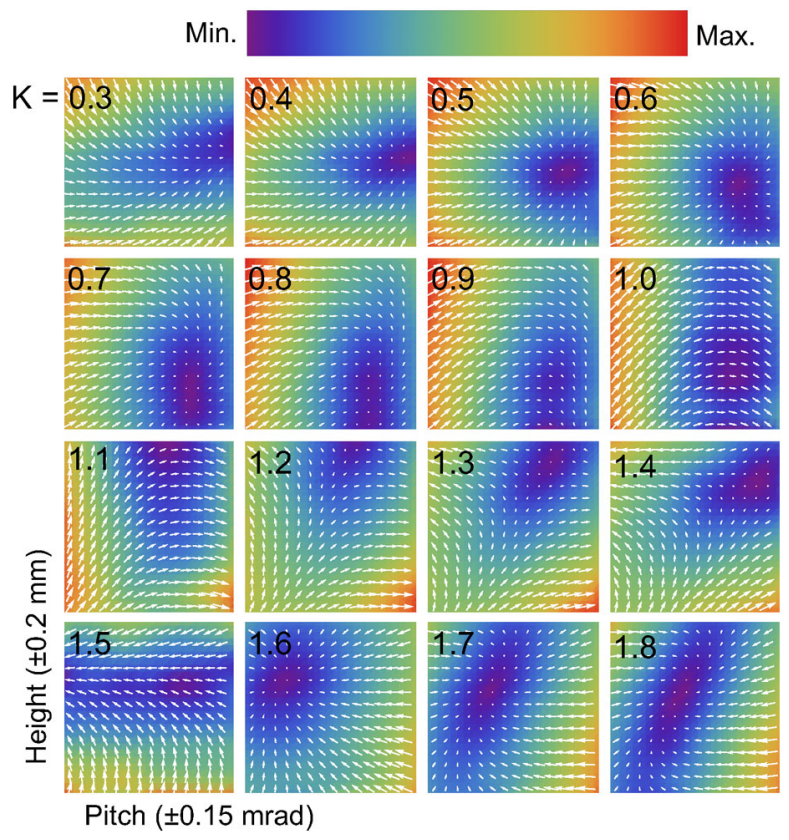

FIG. 68. Position and angle changes of the electron orbit in the vertical plane at the undulator exit $\left(\Delta y\right.$ and $\left.\Delta y^{\prime}\right)$, represented as vertical and horizontal components of small white arrows, as a function of undulator pitch and height, individually plotted for undulator $K$ values ranging from 0.3 to 1.8 . The underlying colors correspond to the lengths of the arrows, i.e., the deviations from zero orbit change, and illustrate the dependence of the natural focusing strength and center on the $K$ value. The plots for the different $K$ values are individually normalized to illustrate the focusing and bending effects.

prohibited the proper propagation of the photon beam in our initial beam-line configuration.

After a modification of the beam line downstream of the undulator, increasing the effective aperture, a round beam spot could be observed on three screens located at distances between 1 and $4 \mathrm{~m}$ from the undulator exit [199]. Due to the temporary failure of the klystron driving the last two Sband structures, all subsequent measurements were performed at beam energies ranging between 100 and $130 \mathrm{MeV}$, using, when needed, the X-band cavity to produce the energy chirp required for bunch compression. At a beam energy of $100 \mathrm{MeV}$, a peak current of $20 \mathrm{~A}$ (obtained without compression) is sufficient to generate FEL radiation with wavelength around $300 \mathrm{~nm}$ for $K=1$, i.e., in the optical (ultraviolet) range. In addition to this "long-bunch" mode, we also compressed the beam to reach a peak current of $200 \mathrm{~A}$ and characterized the FEL radiation resulting from this "short-bunch" mode.

We verified the SASE nature of the observed radiation by confirming two of its salient characteristics, the beam divergence and the statistical distribution of the pulse intensities. To this end we compare the measured properties to simulation results, obtained with the GENESIS 1.3 code [200]. 
A measurement of the photon beam spot size on the first three screens downstream of the undulator yields a photon beam divergence of $0.30 \mathrm{mrad}$, about an order of magnitude below the expected divergence for spontaneous radiation emanating from the undulator, but somewhat lower than the simulation value of $0.38 \mathrm{mrad}$, computed for the same beam conditions (130 MeV beam energy, 200 A peak current). The difference between measurement and simulation is likely related to the uncertainty in the undulator's gain length, which cannot be measured with only one module, and the associated variation of gain guiding effects.

A further distinctive property of SASE radiation is the statistical distribution of the intensities of individual pulses, which is known to follow a single-parameter gamma distribution with free parameter $M$ corresponding to the number of contributing modes [201]. A histogram based on 5000 pulses measured in the long-bunch configuration is shown in the upper plot of Fig. 69, along with a fit to the gamma distribution, which yields a mode parameter $M=136$. Our simulation for this configuration results in $M=208$, with the corresponding curve also indicated in the figure. The measured pulse intensities are based on the signals recorded by the first YAG:Ce screen after the undulator, corrected for background, and assuming reasonable linearity of the YAG:Ce response in the relevant intensity range. The lower plot of Fig. 69 summarizes the analogous data for 1000 pulses obtained in the short-pulse configuration. In this case, the $M$ parameters fitted to the measurement and simulation data are 20 and 28, respectively. The agreement between measured and simulated distributions, even if not perfect, further corroborates our
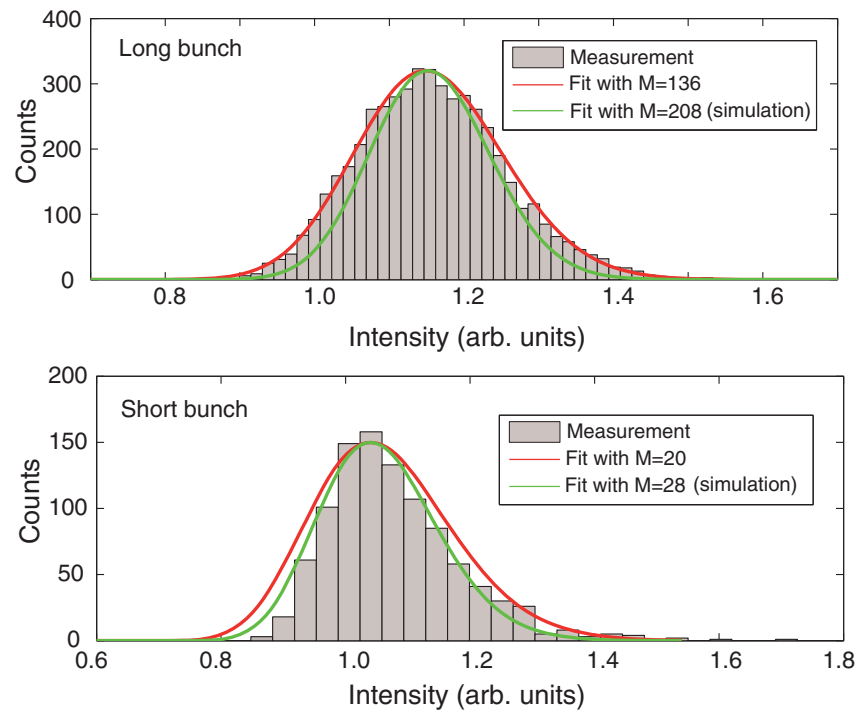

FIG. 69. Intensity distributions of SASE FEL pulses measured in the long-bunch (top) and the short-bunch configuration (bottom), shown as histograms. The curves overlaid represent fits to gamma distributions with the $M$ parameter left free (red) and fixed to simulation results obtained for equivalent beam conditions.

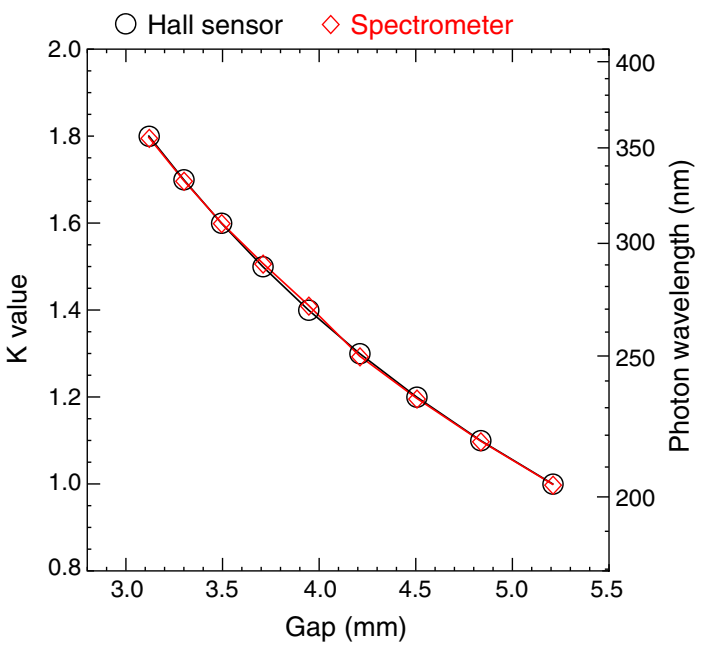

FIG. 70. Undulator $K$ value as a function of undulator gap as derived from magnetic measurements using Hall sensors (black circles) and from spectral measurements of the generated SASE FEL radiation (red diamonds).

interpretation of the observed radiation as originating from the SASE FEL process.

\section{Measurement of $K$ values}

The limitation in beam energy due to the outage of the last S-band booster rf station constrained the FEL spectral range to the optical regime, for which an in-air spectrometer was already available at the SITF. The spectrometer (Ocean Optics QE65000), which had previously been used for studies of optical transition radiation at the same facility [202], covers a spectral range between 220 and $950 \mathrm{~nm}$ wavelength.

At a beam energy of $120 \mathrm{MeV}$, the variation of the undulator gap between 3.0 and $5.5 \mathrm{~mm}$ gives access to a spectral tuning range of the SASE FEL wavelength extending from about 200 to $360 \mathrm{~nm}$. From the measured central wavelengths the corresponding $K$ values of the undulator can be retrieved and compared to the $K$ values derived from the Hall probe measurements on the magnetic bench, see Fig. 70. The observed concurrence bears out the magnetic design and validates the concept of the magnetic measurement bench.

\section{SUMMARY AND OUTLOOK}

We have summarized our experience commissioning and operating the SwissFEL Injector Test Facility during four years, with particular emphasis on system developments and beam physics results relevant for the realization of the SwissFEL facility.

In addition to demonstrating the beam parameters necessary to drive the first stage of an FEL facility, the experience gathered at the SITF has proven invaluable for the evaluation and realization of a UV laser system to drive 
the photoinjector rf gun, the implementation of a stable large-scale rf system, synchronized at the tens of femtosecond level, and the development of numerous diagnostics devices essential for SwissFEL. Furthermore it has enabled the demonstration of a complete undulator system qualified for FEL operation. Beyond the establishment of essential linac and FEL capabilities at PSI the most important advances in view of the larger accelerator community consist in the development of a new profile monitor capable of measuring beams of very low charge and emittance [128], the systematic comparison of intrinsic emittances between cesium telluride and copper cathodes [44], the demonstrated reduction of intrinsic emittance through modifications of the wavelength of the laser driving the electron gun [165] or the gun rf gradient [166], and the development or refinement of various beam characterization methods [148,150,151,156,159].

In terms of beam emittance our efforts resulted in values as low as $100 \mathrm{~nm}$ for bunch charges of $10-20 \mathrm{pC}$ and $200 \mathrm{~nm}$ in the case of $200 \mathrm{pC}$ [148]. These numbers refer to the normalized transverse emittance of individual slices, as relevant for driving FEL facilities (see Sec. VII for details). They have been shown to be reproducible within a few percent and stable under longitudinal bunch compression [187]. At the electron source, we have measured intrinsic emittance values close to the theoretical limit $[44,165,166]$. Overall, the achieved beam quality represents a significant advance in photoinjector physics and technology.

The SITF has also fulfilled an important role as a training facility for machine operators as well as for students of accelerator or FEL-based sciences. The commissioning of the SwissFEL main accelerator and the hard-X-ray undulator and optical lines, scheduled for the years 2016 and 2017 [203], will profit considerably from the experience gained at the SITF.

\section{ACKNOWLEDGMENTS}

We acknowledge the valuable contributions of the entire SwissFEL project staff and all PSI support groups to the design, procurement, production, assembly, installation, validation, and maintenance of the many components and systems that constituted the SwissFEL Injector Test Facility. We also express our thanks to the PSI operations group for helpful assistance with many commissioning tasks. We are indebted to Albin Wrulich for initiating the injector project and for supporting it throughout its earlier stages. Both the SITF design and its operation have much profited from the valuable advice and guidance generously offered by the SwissFEL machine advisory committee (FLAC), chaired by Jörg Roßbach. We further thank Jiaru Shi for help with the tuning of the X-band structure and for providing the corresponding figure, and Nicole Hiller for the careful proofreading of the manuscript. Two detector developments have received financial support from the European Commission under its Seventh
Framework Programme (FP7/2007-2013): the X-band wake-field monitors through the FP7 Research Infrastructures project EuCARD-2, Grant Agreement No. 312453, and the electro-optic bunch length monitor under Grant Agreement No. 290605 (PSI-FELLOW/ COFUND). A part of the beam science program at the SITF was supported by the Swiss National Science Foundation through Grant No. 200021_140659.

[1] SwissFEL conceptual design report, edited by R. Ganter, PSI Report No. 10-04, 2012.

[2] B. D. Patterson et al., Coherent science at the SwissFEL X-ray laser, New J. Phys. 12, 035012 (2010).

[3] SwissFEL injector conceptual design report, edited by $\mathrm{M}$. Pedrozzi, PSI Report No. 10-05, 2010.

[4] J. Rossbach, A VUV free electron laser at the TESLA test facility at DESY, Nucl. Instrum. Methods Phys. Res., Sect. A 375, 269 (1996).

[5] W. Brefeld, B. Faatz, Y. M. Nikitina, J. Pflüger, P. Pierini, J. Roßbach, E. L. Saldin, E. A. Schneidmiller, and M. V. Yurkov, Parameter study on phase I of the VUV-FEL at the TESLA test facility, Nucl. Instrum. Methods Phys. Res., Sect. A 393, 119 (1997).

[6] F. Stephan et al., Detailed characterization of electron sources yielding first demonstration of European X-ray Free Electron Laser beam quality, Phys. Rev. ST Accel. Beams 13, 020704 (2010).

[7] J. F. Schmerge, P. R. Bolton, J. E. Clendenin, F.-J. Decker, D. H. Dowell, S. M. Gierman, C. G. Limborg, and B. F. Murphy, Transverse-emittance measurements on an S-band photocathode RF electron gun, Nucl. Instrum. Methods Phys. Res., Sect. A 483, 301 (2002).

[8] D. Alesini et al., The SPARC project: A high-brightness electron beam source at LNF to drive a SASE-FEL experiment, Nucl. Instrum. Methods Phys. Res., Sect. A 507, 345 (2003).

[9] D. Alesini et al., Status of the SPARC project, Nucl. Instrum. Methods Phys. Res., Sect. A 528, 586 (2004).

[10] Z. T. Zhao, Z. M. Dai, X. F. Zhao, D. K. Liu, Q. G. Zhou, D. H. He, Q. K. Jia, S. Y. Chen, and J.P. Dai, The Shanghai high-gain harmonic generation DUV freeelectron laser, Nucl. Instrum. Methods Phys. Res., Sect. A 528, 591 (2004).

[11] H. Tanaka et al., in Proceedings of the 28th International Free-Electron Laser Conference, Berlin, Germany, 2006 (JACoW, CERN, Geneva, 2006), p. 769.

[12] T. Shintake et al., A compact free-electron laser for generating coherent radiation in the extreme ultraviolet region, Nat. Photonics 2, 555 (2008).

[13] S. J. Park et al., in Proceedings of the 3rd International Particle Accelerator Conference, Shanghai, China, 2013 (JACoW, CERN, Geneva, 2013), p. 2220.

[14] J.-H. Han et al., in Proceedings of the 36th International Free-Electron Laser Conference, Basel, Switzerland, 2014 (JACoW, CERN, Geneva, 2015), p. 615.

[15] J. A. Clarke et al., CLARA conceptual design report, J. Instrum. 9, T05001 (2014). 
[16] R. D. Ruth et al., in Proceedings of the 15th Particle Accelerator Conference, Washington, D.C., USA, 1993 (IEEE, Piscataway, NJ, 1993), p. 543.

[17] D. Xiang et al., Demonstration of the Echo-Enabled Harmonic Generation Technique for Short-Wavelength Seeded Free Electron Lasers, Phys. Rev. Lett. 105, 114801 (2010).

[18] R. Akre et al., Commissioning the Linac Coherent Light Source injector, Phys. Rev. ST Accel. Beams 11, 030703 (2008).

[19] G. Penco et al., Optimization of a high brightness photoinjector for a seeded FEL facility, J. Instrum. 8, P05015 (2013).

[20] Y. Kim et al., in Proceedings of the 11th European Particle Accelerator Conference, Genova, Italy, 2008 (JACoW, CERN, Geneva, 2008), p. 100.

[21] B. E. Carlsten, New photoelectric injector design for the Los Alamos National Laboratory XUV FEL accelerator, Nucl. Instrum. Methods Phys. Res., Sect. A 285, 313 (1989).

[22] L. Serafini and J. B. Rosenzweig, Envelope analysis of intense relativistic quasilaminar beams in $\mathrm{rf}$ photoinjectors: A theory of emittance compensation, Phys. Rev. E 55, 7565 (1997).

[23] M. Ferrario, J. E. Clendenin, D. T. Palmer, J. B. Rosenzweig, and L. Serafini, Report No. SLAC-PUB8400, 2000 (unpublished).

[24] M. Ferrario et al., Direct Measurement of the Double Emittance Minimum in the Beam Dynamics of the Sparc High-Brightness Photoinjector, Phys. Rev. Lett. 99, 234801 (2007).

[25] P. Emma et al., First lasing and operation of an ångstromwavelength free-electron laser, Nat. Photonics 4, 641 (2010).

[26] R. Bossart, H. Braun, M. Dehler, and J.-C. Godot, A $3 \mathrm{GHz}$ photoelectron gun for high beam intensity, Nucl. Instrum. Methods Phys. Res., Sect. A 375, ABS7 (1996).

[27] R. Bossart and M. Dehler, in Proceedings of the 5th European Particle Accelerator Conference, Sitges, Spain, 1996 (IOP, Bristol, 1996), p. 1544.

[28] J.-Y. Raguin, M. Bopp, A. Citterio, and A. Scherer, in Proceedings of the 26th Linear Accelerator Conference, Tel Aviv, Israel, 2012 (JACoW, CERN, Geneva, 2013), p. 442.

[29] J. Schmerge, Report No. SLAC-TN-10-084, 2005 (unpublished).

[30] D. H. Dowell and J. W. Lewellen, in An Engineering Guide To Photoinjectors, edited by T. Rao and D. H. Dowell (Create Space Independent Publishing Platform, North Charlston, SC, 2013), p. 1.

[31] T. Schietinger et al., in Proceedings of the 25th Linear Accelerator Conference, Tsukuba, Japan, 2010 (JACoW, CERN, Geneva, 2011), p. 410.

[32] E. Hohmann, N. Frey, A. Fuchs, C. Harm, H. Hodlmoser, R. Luscher, S. Mayer, O. Morath, R. Philipp, A. Rehmann, and T. Schietinger, Neutron dose rate at the SwissFEL Injector Test Facility: First measurements, Radiation Protection Dosimetry 161, 339 (2014).

[33] E. Hohmann, S. Reiche, A. Fuchs, R. Ganter, F. Le Pimpec, R. Lüscher, S. Mayer, and T. Schietinger, Dose rate produced by secondary radiation at the SwissFEL free electron laser facility, Prog. Nucl. Sci. Technol. 4, 762 (2014).

[34] S. Sanfilippo, M. Negrazus, V. Vrankovic, S. Sidorov, A. Gabard, N. H. Glowa, Y. J. Kim, R. Ganter, M. Pedrozzi, and $\mathrm{H}$. Braun, Magnet design and testing for the $250 \mathrm{MeV}$ injector of the SwissFEL at the Paul Scherrer Institute, IEEE Trans. Appl. Supercond. 20, 265 (2010).

[35] M. Negrazus, A. Gabard, S. Sanfilippo, S. Sidorov, V. Vrankovic, Y. J. Kim, R. Ganter, and M. Pedrozzi, Magnet design and measurement results of the solenoids and bunch compressor bending magnets of the SwissFEL test facility, IEEE Trans. Appl. Supercond. 22, 4101104 (2012).

[36] M. Csatari Divall, M. Kaiser, S. Hunziker, C. Vicario, B. Beutner, T. Schietinger, M. Lüthi, M. Pedrozzi, and C. P. Hauri, Timing jitter studies of the SwissFEL test injector drive laser, Nucl. Instrum. Methods Phys. Res., Sect. A 735, 471 (2014)

[37] A. Trisorio, P. M. Paul, F. Ple, C. Ruchert, C. Vicario, and C. P. Hauri, Ultrabroadband TW-class Ti:sapphire laser system with adjustable central wavelength, bandwidth and multi-color operation, Opt. Express 19, 20128 (2011).

[38] A. Trisorio, C. Ruchert, and C. P. Hauri, Direct shaping of picosecond high energy deep ultraviolet pulses, Appl. Phys. B 105, 255 (2011).

[39] C. Vicario, A. Trisorio, G. Arisholm, and C. P. Hauri, Deep-ultraviolet picosecond flat-top pulses by chirpmatched sum frequency generation, Opt. Lett. 37, 1619 (2012).

[40] G. Suberlucq, CLIC Note No. 299, 1996 (unpublished).

[41] K. Aulenbacher, R. Bossart, H. Braun, J. Clendenin, J. P. Delahaye, J. Madsen, G. Mulhollan, J. Sheppard, G. Suberlucq, and H. Tang, CLIC Note No. 303, 1996 (unpublished).

[42] R. Ganter, M. Bopp, N. Gaiffi, T. Le Quang, M. Pedrozzi, M. Schaer, T. Schietinger, L. Schulz, L. Stingelin, and A. Trisorio, in Proceedings of the 35th International FreeElectron Laser Conference, New York, NY, USA, 2013 (JACoW, CERN, Geneva, 2013), p. 259.

[43] J. Bossert, R. Ganter, M. Schaer, and T. Schietinger, in Proceedings of the 36th International Free-Electron Laser Conference, Basel, Switzerland, 2014 (JACoW, CERN, Geneva, 2015), p. 832.

[44] E. Prat, S. Bettoni, H.-H. Braun, R. Ganter, and T. Schietinger, Measurements of copper and cesium telluride cathodes in a radio-frequency photoinjector, Phys. Rev. ST Accel. Beams 18, 043401 (2015).

[45] M. Dehler, in Proceedings of the 10th International Computational Accelerator Physics Conference, San Francisco, CA, USA, 2009 (JACoW, CERN, Geneva, 2011), p. 285.

[46] R. Losito et al., in Proceedings of the 10th European Particle Accelerator Conference, Edinburgh, Scotland, UK, 2006 (JACoW, CERN, Geneva, 2006), p. 2517.

[47] R. Roux, Conception of photoinjectors for the CTF3 experiment, Int. J. Mod. Phys. A 22, 3925 (2007).

[48] L. Xiao, R. F. Boyce, D. H. Dowell, Z. Li, C. LimborgDeprey, and J. F. Schmerge, in Proceedings of the 21st 
Particle Accelerator Conference, Knoxville, TN, USA, 2005 (IEEE, Piscataway, NJ, 2005), p. 3432.

[49] D. H. Dowell, E. Jongewaard, J. Lewandowski, C. Limborg-Deprey, Z. Li, J. Schmerge, A. Vlieks, J. Wang, and L. Xiao, The development of the Linac Coherent Light Source RF gun, ICFA Beam Dynamics Newsletter 46, 162 (2008).

[50] J. Haimson, B. Mecklenburg, and E. L. Wright, A racetrack geometry to avoid undesirable azimuthal variations of the electric field gradient in high power coupling cavities for TW structures, AIP Conf. Proc. 398, 898 (1997).

[51] J. F. Schmerge, Report No. SLAC-TN-05-027, 2002 (unpublished).

[52] U. Ellenberger et al., in Proceedings of the 27th Linear Accelerator Conference, Geneva, Switzerland, 2014 (JACoW, CERN, Geneva, 2014), p. 1117.

[53] S. Bettoni, P. Craievich, M. Pedrozzi, S. Reiche, and L. Stingelin, in Proceedings of the 35th International FreeElectron Laser Conference, New York, NY, USA, 2013 (JACoW, CERN, Geneva, 2013), p. 209.

[54] P. Craievich et al., in Proceedings of the 36th International Free-Electron Laser Conference, Basel, Switzerland, 2014 (JACoW, CERN, Geneva, 2015), p. 843.

[55] R. H. Fowler and L. Nordheim, Electron emission in intense electric fields, Proc. R. Soc. A 119, 173 (1928).

[56] J.-Y. Raguin, in Proceedings of the 26th Linear Accelerator Conference, Tel Aviv, Israel, 2012 (JACoW, CERN, Geneva, 2013), p. 498.

[57] M. Dehler, J.-Y. Raguin, A. Citterio, A. Falone, W. Wuensch, G. Riddone, A. Grudiev, and R. Zennaro, X-band rf structure with integrated alignment monitors, Phys. Rev. ST Accel. Beams 12, 062001 (2009).

[58] M. Dehler, S. Atieh, D. Gudkov, S. Lebet, G. Riddone, J. Shi, A. Citterio, R. Zennaro, G. D'Auria, and C. Serpico, in Proceedings of the 2nd International Particle Accelerator Conf., San Sebastián, Spain, 2011 (JACoW, CERN, Geneva, 2011), p. 86.

[59] M. Dehler, R. Zennaro, A. Citterio, S. Lebet, G. Riddone, J. Shi, A. Samoshkin, D. Gudkov, G. d'Auria, and C. Serpico, in Proceedings of the 3rd International Particle Accelerator Conference, New Orleans, LA, USA, 2012 (JACoW, CERN, Geneva, 2012), p. 70.

[60] J. Shi, A. Grudiev, and W. Wuensch, Tuning of X-band traveling-wave accelerating structures, Nucl. Instrum. Methods Phys. Res., Sect. A 704, 14 (2013).

[61] Z. Li, in 9th KEK-SLAC ISG Meeting on linear colliders, Tsukuba, Japan, 2002 (unpublished).

[62] C. Nantista, S. Tantawi, and V. Dolgashev, Low-field accelerator structure couplers and design techniques, Phys. Rev. ST Accel. Beams 7, 072001 (2004).

[63] D. Sprehn, A. Haase, A. Jensen, E. N. Jongewaard, C. D. Nantista, S. Tantawi, and A. Vlieks, in Proceedings of the 1st International Particle Accelerator Conference, Kyoto, Japan, 2010 (JACoW, CERN, Geneva, 2010), p. 4020.

[64] G. Caryotakis, in Proceedings of the 17th Particle Accelerator Conference, Vancouver, Canada, 1997 (IEEE, Piscataway, NJ, 1998), p. 2894.
[65] G. A. Loew and O. H. Altenmueller, in Proceedings of the 5th International Conference on High-Energy Accelerators, Frascati, Italy, 1965 (Comitato Naz. Ener. Nucl., Rome, 1966), p. 551.

[66] R. Akre, L. Bentson, P. Emma, and P. Krejcik, in Proceedings of the 19th Particle Accelerator Conference, Chicago, IL, USA, 2001 (IEEE, Piscataway, NJ, 2001), p. 2353.

[67] R. Akre, L. Bentson, P. Emma, and P. Krejcik, in Proceedings of the 8th European Particle Accelerator Conference, Paris, France, 2002 (EPS and CERN, Geneva, 2002), p. 1882.

[68] D. Alesini, G. Di Pirro, L. Ficcadenti, A. Mostacci, L. Palumbo, J. Rosenzweig, and C. Vaccarezza, RF deflector design and measurements for the longitudinal and transverse phase space characterization at SPARC, Nucl. Instrum. Methods Phys. Res., Sect. A 568, 488 (2006).

[69] A. Falone et al., in Proceedings of the 23rd Particle Accelerator Conference, Vancouver, Canada, 2009 (TRIUMF, Vancouver, Canada, 2011), p. 2012.

[70] T. Schilcher, in Proceedings of the CERN Accelerator School on Digital Signal Processing, Sigtuna, Sweden, 2007 (CERN, Geneva, 2008), p. 249.

[71] Z. Geng et al., in Proceedings of the 27th Linear Accelerator Conference, Geneva, Switzerland, 2014 (JACoW, CERN, Geneva, 2014), p. 1114.

[72] A. Hauff et al., in Proceedings of the 27th Linear Accelerator Conference, Geneva, Switzerland, 2014 (JACoW, CERN, Geneva, 2014), p. 683.

[73] M. W. Guetg, B. Beutner, E. Prat, and S. Reiche, Optimization of free electron laser performance by dispersion-based beam-tilt correction, Phys. Rev. ST Accel. Beams 18, 030701 (2015).

[74] J. Welch, S. DeBarger, P. Emma, N. Li, and J. Wu, in Proceedings of the 30th International Free-Electron Laser Conference, Gyeongju, Korea, 2008 (JACoW, CERN, Geneva, 2009), p. 313.

[75] R. Ischebeck et al., in Proceedings of the 3rd International Beam Instrumentation Conference, Monterey, CA, USA, 2014 (JACoW, CERN, Geneva, 2015), p. 119.

[76] M. Dach, M. Dehler, A. Jaggi, R. Kramert, M. Pedrozzi, V. Schlott, and A. Streun, SLS linac diagnosticscommissioning results, AIP Conf. Proc. 546, 563 (2000).

[77] B. Keil, A. Citterio, M. Dehler, R. Ditter, V. Schlott, L. Schulz, and D. M. Treyer, in Proceedings of the 32nd International Free-Electron Laser Conference, Malmö, Sweden, 2010 (JACoW, CERN, Geneva, 2011), p. 429.

[78] D. M. Treyer, R. Baldinger, R. Ditter, B. Keil, W. Koprek, G. Marinkovic, M. Roggli, D. Lipka, D. Noelle, and S. Vilcins, in Proceedings of the 2nd International Beam Instrumentation Conference, Oxford, UK, 2013 (JACoW, CERN, Geneva, 2013), p. 723.

[79] B. Keil et al., in Proceedings of the 2nd International Beam Instrumentation Conference, Oxford, UK, 2013 (JACoW, CERN, Geneva, 2013), p. 427.

[80] A. Citterio, M. Dehler, D. M. Treyer, B. Keil, V. Schlott, and L. Schulz, in Proceedings of the 9th European Workshop on Beam Diagnostics and Instrumentation 
for Particle Accelerators, Basel, Switzerland, 2009 (JACoW, CERN, Geneva, 2009), p. 71.

[81] W. Koprek, R. Baldinger, R. Ditter, B. Keil, G. Marinkovic, M. Roggli, and M. Stadler, in Proceedings of the 1st International Beam Instrumentation Conference, Tsukuba, Japan, 2012 (JACoW, CERN, Geneva, 2013), p. 399.

[82] B. Keil, P.-A. Duperrex, and U. Mueller, in Proceedings of the 10th European Particle Accelerator Conference, Edinburgh, Scotland, UK, 2006 (JACoW, CERN, Geneva, 2006), p. 1226.

[83] J. Adam et al., The MEG detector for $\mu^{+} \rightarrow e^{+} \gamma$ decay search, Eur. Phys. J. C 73, 2365 (2013).

[84] S. Ritt, R. Dinapoli, and U. Hartmann, Application of the DRS chip for fast waveform digitizing, Nucl. Instrum. Methods Phys. Res., Sect. A 623, 486 (2010).

[85] R. Ischebeck, B. Beutner, G. L. Orlandi, M. Pedrozzi, T. Schietinger, V. Schlott, and V. Thominet, in Proceedings of the 25th Linear Accelerator Conference, Tsukuba, Japan, 2010 (JACoW, CERN, Geneva, 2011), p. 656.

[86] H. Loos et al., in Proceedings of the 30th International Free-Electron Laser Conference, Gyeongju, Korea, 2008 (JACoW, CERN, Geneva, 2009), p. 485.

[87] S. Wesch, C. Behrens, B. Schmidt, and P. Schmüser, in Proceedings of the 31st International Free-Electron Laser Conference, Liverpool, UK, 2009 (JACoW, CERN, Geneva, 2009), p. 619.

[88] H. Tanaka, in Proceedings of the 2nd International Particle Accelerator Conference, San Sebastián, Spain, 2011 (JACoW, CERN, Geneva, 2011), p. 21.

[89] A.H. Lumpkin, Report No. FERMILAB-CONF-14105-AD, 2014 (unpublished).

[90] P. Craievich, R. Ischebeck, F. Loehl, G. L. Orlandi, and E. Prat, in Proceedings of the 35th International FreeElectron Laser Conference, New York, NY, USA, 2013 (JACoW, CERN, Geneva, 2013), p. 236.

[91] S. Bettoni, P. Craievich, A. A. Lutman, and M. Pedrozzi, Temporal profile measurements of relativistic electron bunch based on wakefield generation, Phys. Rev. Accel. Beams 19, 021304 (2016).

[92] M. Dach et al., in Proceedings of the 13th International Conference on Accelerator and Large Experimental Physics Control Systems, Grenoble, France, 2011 (JACoW, CERN, Geneva, 2012), p. 593.

[93] P. Chevstov et al., in Proceedings of the 14th International Conference on Accelerator and Large Experimental Physics Control Systems, San Francisco, CA, USA, 2013 (JACoW, CERN, Geneva, 2014), p. 378.

[94] S. Hunt et al., in Proceedings of the 8th International Conference on Accelerator and Large Experimental Physics Control Systems, San Jose, CA, USA, 2001 (SLAC, Stanford, USA, 2002), p. 11.

[95] L. R. Dalesio, J. O. Hill, M. Kraimer, S. Lewis, D. Murray, S. Hunt, W. Watson, M. Clausen, and J. Dalesio, The experimental physics and industrial control system architecture: Past, present, and future, Nucl. Instrum. Methods Phys. Res., Sect. A 352, 179 (1994).

[96] A. Mezger and H. Brands, in Proceedings of the 14th International Conference on Accelerator and Large
Experimental Physics Control Systems, San Francisco, CA, USA, 2013 (JACoW, CERN, Geneva, 2014), p. 864.

[97] J. Chrin, in Proceedings of the 14th International Conference on Accelerator and Large Experimental Physics Control Systems, San Francisco, CA, USA, 2013 (JACoW, CERN, Geneva, 2014), p. 453.

[98] J. Chrin and M. Sloan, in Proceedings of the 13th International Conference on Accelerator and Large Experimental Physics Control Systems, Grenoble, France, 2011 (JACoW, CERN, Geneva, 2012), p. 840.

[99] J. Chrin, in Proceedings of the 15th International Conference on Accelerator and Large Experimental Physics Control Systems, Melbourne, Australia, 2015 (JACoW, CERN, Geneva, 2016), p. 1013.

[100] K. Evans, in Proceedings of the 10th International Conference on Accelerator and Large Experimental Physics Control Systems, Geneva, Switzerland, 2005 (JACoW, CERN, Geneva, 2006), PO1.033-6.

[101] B. Keil, R. Baldinger, R. Ditter, W. Koprek, R. Kramert, G. Marinkovic, M. Roggli, M. Stadler, and D. M. Treyer, in Proceedings of the 1st Int. Beam Instrumentation Conference, Tsukuba, Japan, 2012 (JACoW, CERN, Geneva, 2013), p. 11.

[102] T. Korhonen et al., in Proceedings of the 14th International Conference on Accelerator and Large Experimental Physics Control Systems, San Francisco, CA, USA, 2013 (JACoW, CERN, Geneva, 2014), p. 1032.

[103] R. Krempaská, A. Bertrand, and H. Lutz, in Proceedings of the 14th International Conference on Accelerator and Large Experimental Physics Control Systems, San Francisco, CA, USA, 2013 (JACoW, CERN, Geneva, 2014), p. 1125 .

[104] B. Kalantari and T. Korhonen, in Proceedings of the 9th International Conference on Accelerator and Large Experimental Physics Control Systems, Gyeongju, Korea, 2003 (Pohang Accelerator Lab, Pohang, Korea, 2004), p. 136 .

[105] B. Kalantari and T. Korhonen, in Proceedings of the 13th International Conference on Accelerator and Large Experimental Physics Control Systems, Grenoble, France, 2011 (JACoW, CERN, Geneva, 2012), p. 180.

[106] S. Hunziker, V. Arsov, F. Buechi, M. Kaiser, A. Romann, and V. Schlott, in Proceedings of the 3rd International Beam Instrumentation Conference, Monterey, CA, USA, 2014 (JACoW, CERN, Geneva, 2015), p. 29.

[107] S. Hunziker and V. Schlott, in Proceedings of the 9th European Workshop on Beam Diagnostics and Instrumentation for Particle Accelerators, Basel, Switzerland, 2009 (JACoW, CERN, Geneva, 2009), p. 266.

[108] P. Orel, E. Janezic, P. Lemut, S. Zorzut, S. G. Hunziker, and V. Schlott, in Proceedings of the 5th International Particle Accelerator Conference, Dresden, Germany, 2014 (JACoW, CERN, Geneva, 2014), p. 1751.

[109] V. Arsov, S. Hunziker, M. Kaiser, V. Schlott, and F. Loehl, in Proceedings of the 33rd International Free-Electron Laser Conference, Shanghai, China, 2011 (SINAP, Shanghai, 2012), p. 243.

[110] L. Fröhlich, K. Casarin, E. Quai, A. Holmes-Siedle, M. Severgnini, and R. Vidimari, Online monitoring of 
absorbed dose in undulator magnets with RADFET dosimeters at FERMI@Elettra, Nucl. Instrum. Methods Phys. Res., Sect. A 703, 70 (2013).

[111] L. Fröhlich, S. Grulja, and F. Löhl, in Proceedings of the 2nd International Beam Instrumentation Conference, Oxford, UK, 2013 (JACoW, CERN, Geneva, 2013), p. 481 .

[112] R. Ischebeck et al., in Proceedings of the 2nd International Beam Instrumentation Conference, Oxford, UK, 2013 (JACoW, CERN, Geneva, 2013), p. 12.

[113] K. B. Unser, in Proceedings of the 13th Particle Accelerator Conference, Chicago, IL, USA, 1989 (IEEE, Piscataway, NJ, 1989), p. 71.

[114] K. B. Unser, in Proceedings of the 2nd European Particle Accelerator Conference, Nice, France, 1990 (Editions Frontières, Gif-sur-Yvette, France, 1990), p. 786.

[115] Bergoz Instrumentation, Integrating Current Transformer \& Beam Charge Monitor, http://www.bergoz.com/en/ ict-bcm-ihr.

[116] S. Artinian, J. Bergoz, F. Stulle, P. Pollet, and V. Schlott, in Proceedings of the 1st International Beam Instrumentation Conference, Tsukuba, Japan, 2012 (JACoW, CERN, Geneva, 2013), p. 287.

[117] S. Artinian, J. Bergoz, F. Stulle, P. Pollet, and V. Schlott, in Proceedings of the 3rd International Particle Accelerator Conference, Shanghai, China, 2013 (JACoW, CERN, Geneva, 2013), p. 476.

[118] M. Stadler et al., in Proceedings of the 3rd International Beam Instrumentation Conference, Monterey, CA, USA, 2014 (JACoW, CERN, Geneva, 2015), p. 670.

[119] M. Stadler et al., in Proceedings of the 3rd International Beam Instrumentation Conference, Monterey, CA, USA, 2014 (JACoW, CERN, Geneva, 2015), p. 675.

[120] M. Dehler, S. Bettoni, B. Beutner, and G. de Michele, in Proceedings of the 2nd International Beam Instrumentation Conference, Oxford, UK, 2013 (JACoW, CERN, Geneva, 2013), p. 634.

[121] EuCARD-2: Enhanced European Coordination for Accelerator Research \& Development, http://eucard2.web .cern.ch.

[122] M. Dehler, M. Leich, and S. Hunziker, in Proceedings of the 3rd International Beam Instrumentation Conference, Monterey, CA, USA, 2014 (JACoW, CERN, Geneva, 2015), p. 660.

[123] G. L. Orlandi et al., in Proceedings of the 36th International Free-Electron Laser Conference, Basel, Switzerland, 2014 (JACoW, CERN, Geneva, 2015), p. 948 .

[124] G. Penco et al., in Proceedings of the 37th International Free-Electron Laser Conference, Daejeon, Korea, 2015 (JACoW, CERN, Geneva, 2016), p. 101.

[125] G. L. Orlandi et al., in Proceedings of the 1st International Beam Instrumentation Conference, Tsukuba, Japan, 2012 (JACoW, CERN, Geneva, 2013), p. 271.

[126] G. L. Orlandi et al., in Proceedings of the 2nd International Beam Instrumentation Conference, Oxford, UK, 2013 (JACoW, CERN, Geneva, 2013), p. 515.
[127] K. Floettmann, ASTRA-A Space Charge Tracking Algorithm, http://www.desy.de/ mpyflo/Astra_dokumentation.

[128] R. Ischebeck, E. Prat, V. Thominet, and C. Ozkan Loch, Transverse profile imager for ultra-bright electron beams, Phys. Rev. ST Accel. Beams 18, 082802 (2015).

[129] R. Ischebeck, E. Prat, V. Schlott, V. Thominet, M. Yan, P. Krejcik, and H. Loos, in Proceedings of the 3rd International Beam Instrumentation Conference, Monterey, CA, USA, 2014 (JACoW, CERN, Geneva, 2015), p. 259.

[130] R. Ischebeck, G. L. Orlandi, P. Peier, V. Schlott, B. Smit, C. Vicario, C. Zimmerli, and C. Gerth, in Proceedings of the 33rd International Free-Electron Laser Conference, Shanghai, China, 2011 (SINAP, Shanghai, 2012), p. 572.

[131] F. Frei, I. Gorgisyan, B. Smit, G. L. Orlandi, B. Beutner, E. Prat, R. Ischebeck, V. Schlott, and P. Peier, in Proceedings of the 2nd International Beam Instrumentation Conference, Oxford, UK, 2013 (JACoW, CERN, Geneva, 2013), p. 769

[132] Ch. Gerth, B. Schmidt, S. Wesch, R. Ischebeck, G. L. Orlandi, P. Peier, and V. Schlott, in Proceedings of the 10th European Workshop on Beam Diagnostics and Instrumentation for Particle Accelerators, Hamburg, Germany, 2011 (DESY, Hamburg, 2011), p. 374.

[133] F. Frei et al., in Proceedings of the 36th International Free-Electron Laser Conference, Basel, Switzerland, 2014 (JACoW, CERN, Geneva, 2015), p. 657.

[134] F. Müller, P. Peier, V. Schlott, and B. Steffen, in Proceedings of the 32nd International Free-Electron Laser Conference, Malmö, Sweden, 2010 (JACoW, CERN, Geneva, 2011), p. 374.

[135] F. L. Müller, Ph.D. thesis, Bern University, 2011.

[136] Y. Ivaniskenko, V. Schlott, and P. Peier, in Proceedings of the 36th International Free-Electron Laser Conference, Basel, Switzerland, 2014 (JACoW, CERN, Geneva, 2015), p. 922.

[137] V. Arsov, M. Dehler, S. Hunziker, M. Kaiser, and V. Schlott, in Proceedings of the 2nd International Beam Instrumentation Conference, Oxford, UK, 2013 (JACoW, CERN, Geneva, 2013), p. 8.

[138] V. Arsov, M. Aiba, M. Dehler, F. Frei, S. Hunziker, M. Kaiser, A. Romann, and V. Schlott, in Proceedings of the 36th International Free-Electron Laser Conference, Basel, Switzerland, 2014 (JACoW, CERN, Geneva, 2015), p. 933.

[139] F. Löhl et al., Electron Bunch Timing with Femtosecond Precision in a Superconducting Free-Electron Laser, Phys. Rev. Lett. 104, 144801 (2010).

[140] J. Kim, J. A. Cox, J. Chen, and F. X. Kärtner, Drift-free femtosecond timing synchronization of remote optical and microwave sources, Nat. Photonics 2, 733 (2008).

[141] C. Ozkan Loch, D. Llorente Sancho, P. Pollet, G. Marinkovic, R. Ischebeck, and V. Schlott, in Proceedings of the 4th International Beam Instrumentation Conference, Melbourne, Australia, 2015 (JACoW, CERN, Geneva, 2016), p. 170.

[142] A. Angelovski, A. Kuhl, M. Hansli, A. Penirschke, S. M. Schnepp, M. Bousonville, H. Schlarb, M. K. Bock, T. Weiland, and R. Jakoby, High bandwidth pickup design 
for bunch arrival-time monitors for free-electron laser, Phys. Rev. ST Accel. Beams 15, 112803 (2012).

[143] A. Angelovski et al., Evaluation of the cone-shaped pickup performance for low charge sub-10 fs arrival-time measurements at free electron laser facilities, Phys. Rev. ST Accel. Beams 18, 012801 (2015).

[144] M. Csatari Divall, A. Romann, P. Mutter, S. Hunziker, and C. P. Hauri, in Proceedings of SPIE Advances X-ray FreeElectron Lasers Instr., Prague, Czech Republic, 2015 (International Society for Optics and Photonics, Bellingham, WA, 2015), p. 95121T, http://dx.doi.org/10.1117/12 .2179016 .

[145] M. Krasilnikov, J. Bähr, H.-J. Grabosch, J. H. Han, V. Miltchev, A. Oppelt, B. Petrosyan, L. Staykov, F. Stephan, and M. v. Hartrott, in Proceedings of the 21st Particle Accelerator Conference, Knoxville, TN, USA, 2005 (IEEE, Piscataway, NJ, 2005), p. 967.

[146] T. O. Raubenheimer and R. D. Ruth, A dispersion-free trajectory correction technique for linear colliders, Nucl. Instrum. Methods Phys. Res., Sect. A 302, 191 (1991).

[147] J. Safranek, Experimental determination of storage ring optics using orbit response measurements, Nucl. Instrum. Methods Phys. Res., Sect. A 388, 27 (1997).

[148] E. Prat, M. Aiba, S. Bettoni, B. Beutner, S. Reiche, and T. Schietinger, Emittance measurements and minimization at the SwissFEL Injector Test Facility, Phys. Rev. ST Accel. Beams 17, 104401 (2014).

[149] M. Minty and F. Zimmermann, Measurement and Control of Charged Particle Beams (Springer, Berlin, 2003).

[150] E. Prat, Symmetric single-quadrupole-magnet scan method to measure the $2 \mathrm{D}$ transverse beam parameters, Nucl. Instrum. Methods Phys. Res., Sect. A 743, 103 (2014).

[151] E. Prat and M. Aiba, Four-dimensional transverse beam matrix measurement using the multiple-quadrupole scan technique, Phys. Rev. ST Accel. Beams 17, 052801 (2014).

[152] P. Tenenbaum, CLIC Report No. 326, 1997 (unpublished).

[153] B. Beutner and E. Prat, in Proceedings of the 33rd International Free-Electron Laser Conference, Shanghai, China, 2011 (SINAP, Shanghai, 2012), p. 231.

[154] B. Beutner, R. Ischebeck, and T. Schietinger, in Proceedings of the 25th Linear Accelerator Conference, Tsukuba, Japan, 2010 (JACoW, CERN, Geneva, 2011), p. 653.

[155] B. Beutner, in Proceedings of the 31st International FreeElectron Laser Conference, Liverpool, UK, 2009 (JACoW, CERN, Geneva, 2009), p. 111.

[156] M. Aiba, B. Beutner, and N. Milas, Beam-size-free optics determination, Nucl. Instrum. Methods Phys. Res., Sect. A 753, 24 (2014).

[157] X. Qiu, K. Batchelor, I. Ben-Zvi, and X-J. Wang, Demonstration of Emittance Compensation through the Measurement of the Slice Emittance of a 10-ps Electron Bunch, Phys. Rev. Lett. 76, 3723 (1996).

[158] D. H. Dowell, P. R. Bolton, J. E. Clendenin, P. Emma, S. M. Gierman, C. G. Limborg, B. F. Murphy, J. F. Schmerge, and W.S. Graves, Slice emittance measurements at the SLAC gun test facility, Nucl. Instrum. Methods Phys. Res., Sect. A 507, 327 (2003).
[159] E. Prat and M. Aiba, General and efficient dispersionbased measurement of beam slice parameters, Phys. Rev. ST Accel. Beams 17, 032801 (2014).

[160] K.-J. Kim, Brightness, coherence and propagation characteristics of synchrotron radiation, Nucl. Instrum. Methods Phys. Res., Sect. A 246, 71 (1986).

[161] D. H. Dowell and J. F. Schmerge, Quantum efficiency and thermal emittance of metal photocathodes, Phys. Rev. ST Accel. Beams 12, 074201 (2009).

[162] K. Flöttmann, TESLA FEL Report No. 1997-01, 1997 (unpublished).

[163] D. H. Dowell, I. Bazarov, B. Dunham, K. Harkay, C. Hernandez-Garcia, R. Legg, H. Padmore, T. Rao, J. Smedley, and W. Wan, Cathode R\&D for future light sources, Nucl. Instrum. Methods Phys. Res., Sect. A 622, 685 (2010).

[164] Z. M. Yusof, M.E. Conde, and W. Gai, SchottkyEnabled Photoemission in a rf Accelerator Photoinjector: Possible Generation of Ultralow Transverse ThermalEmittance Electron Beam, Phys. Rev. Lett. 93, 114801 (2004).

[165] M. Csatari Divall, E. Prat, S. Bettoni, C. Vicario, A. Trisorio, T. Schietinger, and C. P. Hauri, Intrinsic emittance reduction of copper cathodes by laser wavelength tuning in an rf photoinjector, Phys. Rev. ST Accel. Beams 18, 033401 (2015).

[166] E. Prat, S. Bettoni, H.-H. Braun, M. Csatari Divall, and T. Schietinger, Measurements of intrinsic emittance dependence on rf field for copper photocathodes, Phys. Rev. ST Accel. Beams 18, 063401 (2015).

[167] C. P. Hauri, R. Ganter, F. Le Pimpec, A. Trisorio, C. Ruchert, and H. H. Braun, Intrinsic Emittance Reduction of an Electron Beam from Metal Photocathodes, Phys. Rev. Lett. 104, 234802 (2010).

[168] R. Ganter et al., Electron beam characterization of a combined diode rf electron gun, Phys. Rev. ST Accel. Beams 13, 093502 (2010).

[169] W. S. Graves, L. F. DiMauro, R. Heese, E. D. Johnson, J. Rose, J. Rudati, T. Shaftan, and B. Sheehy, in Proceedings of the 19th Particle Accelerator Conference, Chicago, IL, USA, 2001 (IEEE, Piscataway, NJ, 2001), p. 2227.

[170] J. F. Schmerge, J. M. Castro, J. E. Clendenin, D. H. Dowell, S. M. Gierman, and R. O. Hette, in Proceedings of the 26th International Free-Electron Laser Conference and 11th FEL Users Workshop, Trieste, Italy, 2004 (Comitato Conferenze Elettra, Trieste, 2005), p. 205.

[171] Y. Ding et al., Measurements and Simulations of Ultralow Emittance and Ultrashort Electron Beams in the Linac Coherent Light Source, Phys. Rev. Lett. 102, 254801 (2009).

[172] H. J. Qian, C. Li, Y.C. Du, L.X. Yan, J. F. Hua, W. H. Huang, and C. X. Tang, Experimental investigation of thermal emittance components of copper photocathode, Phys. Rev. ST Accel. Beams 15, 040102 (2012)

[173] D. Sertore, D. Favia, P. Michelato, L. Monaco, and P. Pierini, in Proceedings of the 9th European Particle Accelerator Conference, Lucerne, Switzerland, 2004 (EPS and CERN, Geneva, 2004), p. 408. 
[174] S. Bettoni, M. Pedrozzi, and S. Reiche, Low emittance injector design for free electron lasers, Phys. Rev. ST Accel. Beams 18, 123403 (2015).

[175] M. Dohlus, T. Limberg, and P. Emma, Electron bunch length compression, ICFA Beam Dynamics Newsletter 38, 15 (2005).

[176] P. Emma, in Handbook of Accelerator Physics and Engineering, Second Edition, edited by A. W. Chao, K. H. Mess, M. Tigner, and F. Zimmermann (World Scientific, Singapore, 2013), p. 334.

[177] I. Zagorodnov and M. Dohlus, Semianalytical modeling of multistage bunch compression with collective effects, Phys. Rev. ST Accel. Beams 14, 014403 (2011).

[178] W. S. Graves et al., in Proceedings of the 19th Particle Accelerator Conference, Chicago, IL, USA, 2001 (IEEE, Piscataway, NJ, 2001), p. 2224.

[179] T. Shaftan and Z. Huang, Experimental characterization of a space charge induced modulation in high-brightness electron beam, Phys. Rev. ST Accel. Beams 7, 080702 (2004).

[180] M. Hüning, Ph. Piot, and H. Schlarb, Observation of longitudinal phase space fragmentation at the TESLA test facility free-electron laser, Nucl. Instrum. Methods Phys. Res., Sect. A 475, 348 (2001).

[181] Z. Huang, J. Wu, and T. Shaftan, Microbunching instability due to bunch compression, ICFA Beam Dynamics Newsletter 38, 37 (2005).

[182] M. Pedrozzi, M. Calvi, R. Ischebeck, S. Reiche, C. Vicario, B. D. Fell, and N. Thompson, in Proceedings of the 36th International Free-Electron Laser Conference, Basel, Switzerland, 2014 (JACoW, CERN, Geneva, 2015), p. 871.

[183] Z. Huang, M. Borland, P. Emma, J. Wu, C. Limborg, G. Stupakov, and J. Welch, Suppression of microbunching instability in the linac coherent light source, Phys. Rev. ST Accel. Beams 7, 074401 (2004).

[184] Z. Huang et al., Measurements of the linac coherent light source laser heater and its impact on the x-ray freeelectron laser performance, Phys. Rev. ST Accel. Beams 13, 020703 (2010).

[185] M. Röhrs, C. Gerth, M. Hüning, and H. Schlarb, in Proceedings of the 10th European Particle Accelerator Conference, Edinburgh, Scotland, UK, 2006, (JACoW, CERN, Geneva, 2006), p. 77.

[186] F. Zhou, K. Bane, Y. Ding, Z. Huang, H. Loos, and T. Raubenheimer, Measurements and analysis of a highbrightness electron beam collimated in a magnetic bunch compressor, Phys. Rev. ST Accel. Beams 18, 050702 (2015).

[187] S. Bettoni, M. Aiba, B. Beutner, M. Pedrozzi, E. Prat, S. Reiche, and T. Schietinger, Preservation of low slice emittance in bunch compressors, Phys. Rev. Accel. Beams 19, 034402 (2016).
[188] M. Dohlus and T. Limborg, CSRTrack, http://www.desy .de/xfel-beam/csrtrack/.

[189] M. Dohlus, TESLA FEL Report No. 2003-05, 2003 (unpublished).

[190] M. Yan, Ch. Gerth, B. Beutner, R. Ischebeck, and E. Prat, in Proceedings of the 36th International Free-Electron Laser Conference, Basel, Switzerland, 2014 (JACoW, CERN, Geneva, 2015), p. 941.

[191] T. Schmidt, G. Ingold, A. Imhof, B. D. Patterson, L. Patthey, C. Quitmann, C. Schulze-Briese, and R. Abela, Insertion devices at the Swiss Light Source (phase I), Nucl. Instrum. Methods Phys. Res., Sect. A 467-468, 126 (2001).

[192] T. Schmidt, M. Calvi, and G. Ingold, Undulators for the PSI light sources, Synchrotron Radiat. News 28, 34 (2015).

[193] T. Schmidt and S. Reiche, in Proceedings of the 31st International Free-Electron Laser Conference, Liverpool, UK, 2009 (JACoW, CERN, Geneva, 2009), p. 706.

[194] T. Hara, T. Tanaka, T. Tanabe, X.-M. Maréchal, S. Okada, and H. Kitamura, In-vacuum undulators of Spring-8, J. Synchrotron Radiat. 5, 403 (1998).

[195] M. Calvi, M. Brügger, S. Danner, A. Imhof, H. Jöhri, T. Schmidt, and C. Scoular, in Proceedings of the 34th International Free-Electron Laser Conference, Nara, Japan, 2012 (JACoW, CERN, Geneva, 2013), p. 662.

[196] T. Schmidt et al., in Proceedings of the 34th International Free-Electron Laser Conference, Nara, Japan, 2012 (JACoW, CERN, Geneva, 2013), p. 666.

[197] M. Calvi, M. Aiba, M. Brügger, S. Danner, R. Ganter, C. Ozkan, and Th. Schmidt, in Proceedings of the 36th International Free-Electron Laser Conference, Basel, Switzerland, 2014 (JACoW, CERN, Geneva, 2015), p. 111.

[198] M. Calvi, M. Aiba, M. Brügger, S. Danner, Th. Schmidt, R. Ganter, L. Patthey, Th. Schietinger, and R. Ischebeck, in Proceedings of the 36th International Free-Electron Laser Conference, Basel, Switzerland, 2014 (JACoW, CERN, Geneva, 2015), p. 107.

[199] S. Reiche, in Proceedings of the 36th International FreeElectron Laser Conference, Basel, Switzerland, 2014 (JACoW, CERN, Geneva, 2015), p. 144.

[200] S. Reiche, GENESIS 1.3: a fully 3D time-dependent FEL simulation code, Nucl. Instrum. Methods Phys. Res., Sect. A 429, 243 (1999).

[201] E. L. Saldin, E. A. Schneidmiller, and M. V. Yurkov, Statistical properties of radiation from VUV and X-ray free electron laser, Opt. Commun. 148, 383 (1998).

[202] B. Smit, Ph.D. thesis No. 6570, EPF Lausanne, 2015, http://dx.doi.org/10.5075/epfl-thesis-6570.

[203] T. Schietinger, in Proceedings of the 37th International Free-Electron Laser Conference, Daejeon, Korea, 2015 (JACoW, CERN, Geneva, 2016), p. 69. 